



\section{Nutritional challenges in adult patients with cystic fibrosis pre and post lung transplantation}

Francis Hollander-Kraaijeveld 
Nutritional challenges in adult patients with cystic fibrosis pre and post lung transplantation

ISBN/EAN: 978-90-393-7296-8

Layout and design by David de Groot | www.persoonlijkproefschrift.nl

Printed by Ridderprint | www.ridderprint.nl

(c) F.M. Hollander-Kraaijeveld 2020

All rights reserved. No part of this thesis may be reproduced, stored or transmitted in any way or by any means without the prior permission of the author, or when applicable, of the publishers of the scientific papers.

Dit proefschrift werd (mede) mogelijk gemaakt met financiële steun van COSMED Benelux BV, Eurocept Homecare, Nederlandse Vereniging van Diëtisten, Nederlandse Transplantatie Vereniging, Nutricia Specialized Nutrition, Mediq Nederland B.V., Mylan Healthcare B.V., Sorgente B.V. 


\title{
Nutritional challenges in adult patients with cystic fibrosis pre and post lung transplantation
}

\author{
Uitdagingen in voedingsbeleid bij volwassen patiënten \\ met cystic fibrosis \\ voor en na longtransplantatie
}

\section{Proefschrift}
ter verkrijging van de graad van doctor aan de
Universiteit Utrecht
op gezag van de
rector magnificus, prof.dr. H.R.B.M. Kummeling, ingevolge het besluit van het college voor promoties
in het openbaar te verdedigen op

donderdag 9 juli 2020 des ochtends te 10.30 uur

door

Franziska Magdalena Hollander-Kraaijeveld

geboren op 3 april 1965

te Ridderkerk 


\section{Promotor:}

Prof. dr. H.G.M. Heijerman

\section{Copromotor:}

Dr. N.M. de Roos 
"Your work is going to fill a large part of your life,

and the only way to be truly satisfied is to do what you believe is great work.

The only way to do great work is to love what you do."

Voor Laura, Julia en David

Voor Hans

Voor papa † en mama † 



\section{CONTENTS}

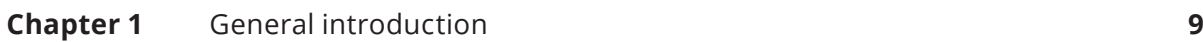

Chapter 2 The optimal approach to nutrition and cystic fibrosis: latest evidence and recommendations

Chapter 3 Prediction equations underestimate resting energy expenditure in patients with end-stage cystic fibrosis

Chapter 4 Non-fasting bioelectrical impedance analysis in cystic fibrosis: Implications for clinical practice and research

Chapter 5 Resting energy expenditure in cystic fibrosis patients decreases after lung transplantation, which improves applicability of prediction equations for energy requirement

Chapter 6 Body weight and body mass index in patients with end-stage cystic fibrosis stabilize after the start of enteral tube feeding

Chapter 7 Effects of nutritional status and dietetic interventions on survival in cystic fibrosis patients before and after lung transplantation

Chapter 8 General Discussion and Summary 
蛋

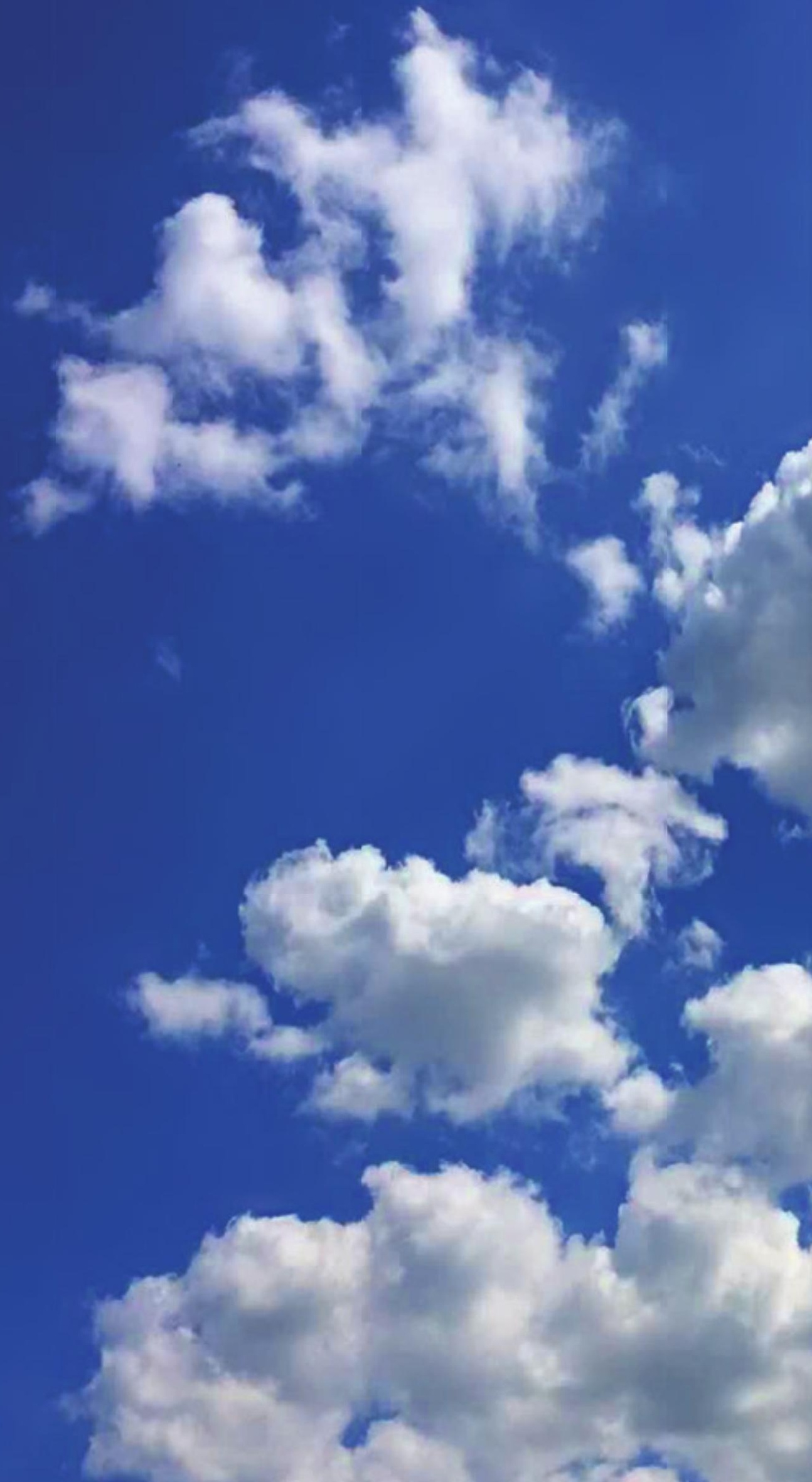




\section{Chapter 1}

General introduction 


\section{CYSTIC FIBROSIS}

Cystic fibrosis (CF) is one of the most common fatal hereditary disorders worldwide. ${ }^{1}$ It is caused by mutations in the cystic fibrosis transmembrane conductance regulator (CFTR) gene. This gene codes for the CFTR protein, which functions as a channel for chloride ions and water across the membrane of cells that produce mucus, sweat, saliva, tears, and digestive enzymes. A mutation in this CFTR gene impairs the function of these channels, which leads to thicker and sticky mucus. Because the CFTR protein is present in all mucus producing organs in the body, CF is a multi-system genetic disorder affecting the lungs, pancreas, liver, intestine and other organs. ${ }^{2,3}$ Symptoms of CF can differ due to the many different mutations in the CFTR gene, ranging from complete absence or mild dysfunction of CFTR. Depending on the impaired function of CFTR, patients may experience fat malabsorption and chronic pulmonary infections leading to bronchiectasis and progressive lung damage. ${ }^{4}$

Therapy is directed to maintain and optimize pulmonary function and nutritional status, since these are key factors in survival. ${ }^{5}$ Recent decades have shown a great improvement in survival of individuals with CF. ${ }^{6}$ Registries in Europe, the USA, and Canada now demonstrate that median survival for CF is around $40-50$ years. ${ }^{7}$ This is much higher than the median age at death worldwide, which is only around 30 years ${ }^{6,7}$ due to health inequalities and a dramatic variation in survival of CF individuals across Europe. ${ }^{6,8}$ The increased survival in some Western countries can be explained by newborn screening programs, ${ }^{9,10}$ centralized care $^{9}$ using evidence-based clinical and nutrition guidelines, ${ }^{11}$ access to CF specific medications, and the introduction of drugs that modulate CFTR. ${ }^{12,13}$ As the majority of children with CF now live into adulthood, the number of adults with CF is growing. This also means that more CF patients have different stages of pulmonary disease, with complex co-morbidity. Therefore, health care professionals need to be able to adapt the nutritional treatment of a CF patient across the life span.

\section{NUTRITIONAL CARE}

Nutritional status has a positive association with pulmonary function and survival in CF. ${ }^{14}$ However, many patients suffer from an impaired nutritional status, due to a combination of increased nutrient demands, increased losses, and impaired nutritional intake. Insufficient nutritional intake is common in the CF population. There are many different reasons for this. First, CF patients often experience severe fatigue ${ }^{15}$ which can contribute to a reduced food 
consumption with increased risk of nutrient deficiencies. ${ }^{16}$ Second, chronic loss of appetite in CF patients is a well known problem ${ }^{17,18}$ without appropriate treatment. ${ }^{19}$ These problems often concerns both individuals and their families, and may contribute to development of complex family or social situations with disturbed eating behaviors. ${ }^{19}$ Moreover, risk factors for developing eating disorders include factors which may be a direct consequence of severe CF disease such as delayed growth and puberty, low body weight, comorbidities such as cystic fibrosis related diabetes (CFRD), preoccupation with the disease and dietary management, as well as the reduced life expectancy. ${ }^{20}$ Also social isolation, low self esteem and a disturbed body image may be a result of CF. ${ }^{21}$ All of these issues, in combination with gastro-intestinal dysfunction and increased caloric expenditure, make it often difficult to attain an appropriate nutritional status. ${ }^{22}$ Increased caloric expenditure is mainly caused by increased work of breathing due to chronic lung infection and loss of pulmonary function. In addition, chronic pulmonary infection and inflammation lead to cytokine-induced catabolism. ${ }^{22}$ To compensate for these demands, nutrition guidelines for patients with CF recommend energy intakes of $110-200 \%$ of requirements for the healthy population of the same sex and age ${ }^{11}$ to maintain or achieve body mass index (BMI) targets. ${ }^{11,23}$ Furthermore, if oral intake is insufficient, enteral tube feeding is described as an effective method to improve nutritional status, especially in malnourished patients. ${ }^{24}$

Despite appropriate management, CF patients still require intensive monitoring for both malnutrition and overweight/obesity. A study in sixty-eight Greek CF patients showed that $22 \%$ of the patients were malnourished and $13 \%$ was overweight or obese. In the remaining $65 \%$ of the patients, only $29 \%$ met the classification of a normal nutritional status based on having a body mass index (BMI) between the $10^{\text {th }}$ and $85^{\text {th }}$ percentiles in children and adolescents or between 18.5 and $24.9 \mathrm{~kg} / \mathrm{m}^{2}$ in adults. ${ }^{25}$ However, even a healthy weight or BMI may mask nutritional problems, as these do not provide insight in the proportion of fat and fat free mass (FFM). Insight in body composition is important, because CF patients with a higher FFM were shown to have a better pulmonary function than patients with a higher FM. ${ }^{26,27}$ Moreover, depleted or low FFM is associated with significant lung disease and impaired pulmonary function. ${ }^{28}$ Losses of both muscle mass and muscle function must be prevented, because they will lead to unwanted weight loss, exhaustion and reduced physical activity capacity resulting in a higher risk of mortality. ${ }^{29-31}$ Therefore, BMI and weight measurements do not detect changes in tissue composition and therefore may underestimate results of nutritional support. ${ }^{32}$ As more patients with CF live well into adulthood, knowledge about the concepts and measurements of body composition has become more clinically important. 


\section{LUNG TRANSPLANTATION}

Despite better medication and nutrition guidelines, most CF patients still need a lung transplantation (LTx) to improve survival. ${ }^{33,34} \mathrm{LTX}$ is a well-established treatment option for CF patients with end-stage lung disease. ${ }^{35,36}$ This therapeutic option should be considered for patients whose clinical status has progressively declined despite maximal medical treatment. International guidelines suggest that transplantation should be considered for patients with CF who have a 2-year predicted survival of less than $50 \%$ and who have functional limitations classified as New York Heart Association Class III or IV. ${ }^{37}$ If FEV1 has fallen to $30 \%$ or a patient with advanced disease shows a rapidly falling FEV1 despite optimal therapy, referral to a lung transplant center is warranted. ${ }^{37}$ Patients are accepted on the waiting list if contra-indications are absent. The decision to list patients with CF is complex and should take into account the rate of decline in pulmonary function, frequency of exacerbations, and the development of baseline hypercapnia and pulmonary hypertension. These current recommendations from the International Society of Heart and Lung Transplantation are based on small studies and expert opinion consensus. ${ }^{34}$

In order to reach the optimal survival rates before and after LTX, the Lung Allocation Score (LAS) has been recently introduced in the Netherlands (2014). LAS is used as marker of disease severity with scores ranging from 0 to 100. A high LAS is reflecting individuals with the most urgent need and the greatest change of success after transplantation. ${ }^{38}$ The use of LAS has been associated with shorter waiting list times and an improved survival benefit. ${ }^{36,38}$ However, CF lung transplant recipients with a high LAS $(>46)$ also show a decreased one year posttransplant survival ${ }^{38}$ and due to a shortage of available donor lungs, a considerable proportion of CF patients with high LAS already die before transplantation. ${ }^{39}$

For LAS calculation, anthropometric parameters such as weight and height are needed..$^{40}$ Ongoing weight loss despite aggressive nutritional supplementation is one of the current criteria for patients to be eligible for lung transplantation. ${ }^{34}$ However, relative contraindications for LTX are a nutritional status with a BMI below 18 or above $30 .{ }^{34,37} \mathrm{It}$ is a challenge for dietitians working in transplant care to optimize energy requirements for improving the nutritional status in CF patients before LTX. One of the challenges is the difficulty in establishing the caloric demands. Indirect calorimetry is the most commonly used and reliable non-invasive method for measuring the REE in clinical populations, also in CF. ${ }^{41}$ A small study performed in ten CF children showed an increased (132\% of predicted) mean resting energy expenditure (REE) measured by indirect calorimetry before LTX that decreased to $112 \%$ of predicted REE 
within one year after LTX. ${ }^{42}$ However, longitudinal studies in which REE before and after LTX is investigated in adult CF patients are lacking.

As in the general CF population, it is warranted to investigate whether BMI is an adequate measure to assess and monitor nutritional status in CF LTx patients, as is now stated in current international guidelines. ${ }^{11}$ Moreover, current diagnostic tools for assessment of the nutritional status need to be explored in CF and also the applicability of these tools in CF LTX patients. Furthermore, research into effective nutritional intervention strategies in CF LTX patients are rare and highly warranted to optimize nutritional status and to contribute to the improvement of lung transplant outcome.

Aims and outline of this thesis This thesis aims to increase the knowledge on body composition, caloric demands, and effectiveness of nutritional interventions in adult CF patients, and how to measure these outcomes. Data were derived from clinical practice, ranging from outpatients who came for regular check-ups to end-stage patients before and after lung transplantation.

Chapter 2 provides a review of literature published between 2015 and 2017 about nutritional management associated with pulmonary function and outcome in cystic fibrosis. Based on this review, which includes expert-based guidelines, optimal nutritional care of CF patients is proposed. In Chapter $\mathbf{3}$ to $\mathbf{5}$ we compare current clinically applicable methods to estimate body composition and resting energy expenditure with actual measurements. In Chapter 3, we put measured versus predicted resting energy expenditures (REE) side by side in a cross-section of end-stage cystic fibrosis patients, using indirect calorimetry and different prediction equations used in clinical practice. In Chapter $\mathbf{4}$ we describe the bioelectrical impedance analysis (BIA) as a method to assess body composition in adult CF patients and we assess whether the differences between fasting and non-fasting BIA results are clinically relevant. The same techniques of BIA and REE are used in a unique longitudinal study in a cohort of CF patients before and after their lung transplantation and those results are presented in Chapter 5. Effectiveness of nutritional interventions is studied in Chapter 6 and $\mathbf{7}$ in two other cohorts. In Chapter $\mathbf{6}$ we show the results of an observational study in which we monitored body weight and body mass index (BMI) before and after the start of enteral tube feeding (ETF) in patients with end-stage cystic fibrosis. Moreover, in Chapter 7, we investigate BMI and fat free mass index (FFMI), dietetic intervention and dietary intake in CF patients before and after lung transplantation. Finally, in Chapter 8, the contents of this thesis will be discussed and summarized. Also recommendations for current clinical practice and future directions for research are indicated. 


\section{REFERENCES}

1. Mall MA and Hartl D. CFTR: cystic fibrosis and beyond. Eur Respir J. 2014;44:1042-54.

2. Villanueva G, Marceniuk G, Murphy MS, Walshaw M, Cosulich R and Guideline C. Diagnosis and management of cystic fibrosis: summary of NICE guidance. BMJ. 2017;359:j4574.

3. Cystic Fibrosis:Diagnosis and management.National Institute for Health and Care Excellence: Clinical Guidelines. London 2017.

4. Castellani C and Assael BM. Cystic fibrosis: a clinical view. Cell Mol Life Sci. 2017;74:129-40.

5. Stephenson AL, Mannik LA, Walsh S, et al. Longitudinal trends in nutritional status and the relation between lung function and BMI in cystic fibrosis: a population-based cohort study. Am J Clin Nutr. 2013;97:872-7.

6. Elborn JS, Bell SC, Madge SL, et al. Report of the European Respiratory Society/European Cystic Fibrosis Society task force on the care of adults with cystic fibrosis. Eur Respir J. 2016; 47:420-8.

7. Salvatore D, Buzzetti R and Mastella G. Update of literature from cystic fibrosis registries 2012 2015. Part 6: Epidemiology, nutrition and complications. Pediatr Pulmonol. 2017;52:390-8.

8. Iskrov GG, Stefanov RS, Lopez-Bastida J, et al. Economic Burden And Health-Related Quality Of Life Of Patients With Cystic Fibrosis In Bulgaria. Folia Med (Plovdiv). 2015;57:56-64.

9. Farrell PM, White TB, Howenstine MS, et al. Diagnosis of Cystic Fibrosis in Screened Populations. J Pediatr. 2017;181S: S33-S44 e2.

10. Tridello G, Castellani C, Meneghelli I, Tamanini A and Assael BM. Early diagnosis from newborn screening maximises survival in severe cystic fibrosis. ERJ Open Res. 2018;4.

11. Turck D, Braegger CP, Colombo C, et al. ESPEN-ESPGHAN-ECFS guidelines on nutrition care for infants, children, and adults with cystic fibrosis. Clin Nutr. 2016;35:557-77.

12. Borowitz D, Lubarsky B, Wilschanski M, et al. Nutritional Status Improved in Cystic Fibrosis Patients with the G551D Mutation After Treatment with Ivacaftor. Dig Dis Sci. 2016;61:198-207.

13. Konstan MW, McKone EF, Moss RB, et al. Assessment of safety and efficacy of long-term treatment with combination lumacaftor and ivacaftor therapy in patients with cystic fibrosis homozygous for the F508del-CFTR mutation (PROGRESS): a phase 3, extension study. Lancet Respir Med. 2017;5:10718.

14. Yen EH, Quinton H and Borowitz D. Better nutritional status in early childhood is associated with improved clinical outcomes and survival in patients with cystic fibrosis. J Pediatr. 2013;162:530$5 e 1$.

15. Nap-van der Vlist MM, Burghard M, Hulzebos HJ, et al. Prevalence of severe fatigue among adults with cystic fibrosis: A single center study. J Cyst Fibros. 2018;17:368-74.

16. $\mathrm{Li} \mathrm{L}$ and Somerset S. Dietary intake and nutritional status of micronutrients in adults with cystic fibrosis in relation to current recommendations. Clin Nutr. 2016;35:775-82.

17. Sullivan JS and Mascarenhas MR. Nutrition: Prevention and management of nutritional failure in Cystic Fibrosis. J Cyst Fibros. 2017 16(suppl 2): S87-S93.

18. Pencharz PB and Durie PR. Pathogenesis of malnutrition in cystic fibrosis, and its treatment. Clin Nutr. 2000;19: 87-94.

19. Chinuck R, Dewar J, Baldwin DR and Hendron E. Appetite stimulants for people with cystic fibrosis. Cochrane Database Syst Rev. 2014:CD008190. 
20. Linkson L, Macedo P, Perrin FMR and Elston CM. Anorexia nervosa in cystic fibrosis. Paediatr Respir Rev. 2018;26:24-6.

21. Pinquart M. Body image of children and adolescents with chronic illness: a meta-analytic comparison with healthy peers. Body Image. 2013;10:141-8.

22. Lusman S and Sullivan J. Nutrition and Growth in Cystic Fibrosis. Pediatr Clin North Am. 2016;63:66178.

23. Stallings VA, Stark LJ, Robinson KA, et al. Evidence-based practice recommendations for nutritionrelated management of children and adults with cystic fibrosis and pancreatic insufficiency: results of a systematic review. J Am Diet Assoc. 2008;108:832-9.

24. Woestenenk JW, Castelijns SJAM, van der Ent CK and Houwen RHJ. Nutritional intervention in patients with Cystic Fibrosis: A systematic review. J Cyst Fibros. 2013;12:102-15.

25. Panagopoulou P, Fotoulaki M, Nikolaou A and Nousia-Arvanitakis S. Prevalence of malnutrition and obesity among cystic fibrosis patients. Pediatr Int. 2014;56:89-94.

26. Bosy-Westphal A and Muller MJ. Identification of skeletal muscle mass depletion across age and $\mathrm{BMI}$ groups in health and disease--there is need for a unified definition. International journal of obesity. 2015;39:379-86.

27. Alvarez JA, Ziegler TR, Millson EC and Stecenko AA. Body composition and lung function in cystic fibrosis and their association with adiposity and normal-weight obesity. Nutrition. 2016 32:447-52.

28. Sheikh S, Zemel BS, Stallings VA, Rubenstein RC and Kelly A. Body composition and pulmonary function in cystic fibrosis. Front Pediatr. 2014;2:33.

29. Yannakoulia M, Ntanasi E, Anastasiou CA and Scarmeas N. Frailty and nutrition: From epidemiological and clinical evidence to potential mechanisms. Metabolism. 2017;68:64-76.

30. Upala S, Panichsillapakit T, Wijarnpreecha K, Jaruvongvanich V and Sanguankeo A. Underweight and obesity increase the risk of mortality after lung transplantation: a systematic review and meta-analysis. Transpl Int. 2016;29:285-96.

31. Cruz-Jentoft AJ, Kiesswetter E, Drey M and Sieber CC. Nutrition, frailty, and sarcopenia. Aging Clin Exp Res. 2017;29:43-8.

32. Collins S. Nutritional management of cystic fibrosis an update for the 21st century. Paediatr Respir Rev. 2017.

33. Yusen RD, Edwards LB, Kucheryavaya AY, et al. The Registry of the International Society for Heart and Lung Transplantation: Thirty-second Official Adult Lung and Heart-Lung Transplantation Report--2015; Focus Theme: Early Graft Failure. J Heart Lung Transplant. 2015;34:1264-77.

34. Morrell MR and Pilewski JM. Lung Transplantation for Cystic Fibrosis. Clin Chest Med. 2016;37:12738.

35. Corris PA. Lung transplantation for cystic fibrosis and bronchiectasis. Semin Respir Crit Care Med. 2013;34:297-304.

36. Thabut G, Christie JD, Mal H, et al. Survival benefit of lung transplant for cystic fibrosis since lung allocation score implementation. Am J Respir Crit Care Med. 2013;187:1335-40.

37. Weill D, Benden C, Corris PA, et al. A consensus document for the selection of lung transplant candidates: 2014--an update from the Pulmonary Transplantation Council of the International Society for Heart and Lung Transplantation. J Heart Lung Transplant. 2015;34:1-15.

38. Braun AT, Dasenbrook EC, Shah AS, Orens JB and Merlo CA. Impact of lung allocation score on survival in cystic fibrosis lung transplant recipients. J Heart Lung Transplant. 2015;34:1436-41. 
39. Martin C, Hamard C, Kanaan R, et al. Causes of death in French cystic fibrosis patients: The need for improvement in transplantation referral strategies! J Cystic Fibros. 2016;15:204-12.

40. Gottlieb J. Lung allocation. J Thorac Dis. 2017;9:2670-4.

41. Psota T and Chen KY. Measuring energy expenditure in clinical populations: rewards and challenges. EurJ Clin Nutr. 2013;67:436-42.

42. Kalnins D, Pencharz PB, Grasemann $\mathrm{H}$ and Solomon M. Energy expenditure and nutritional status in pediatric patients before and after lung transplantation. J Pediatr. 2013;163:1500-2. 
蛋

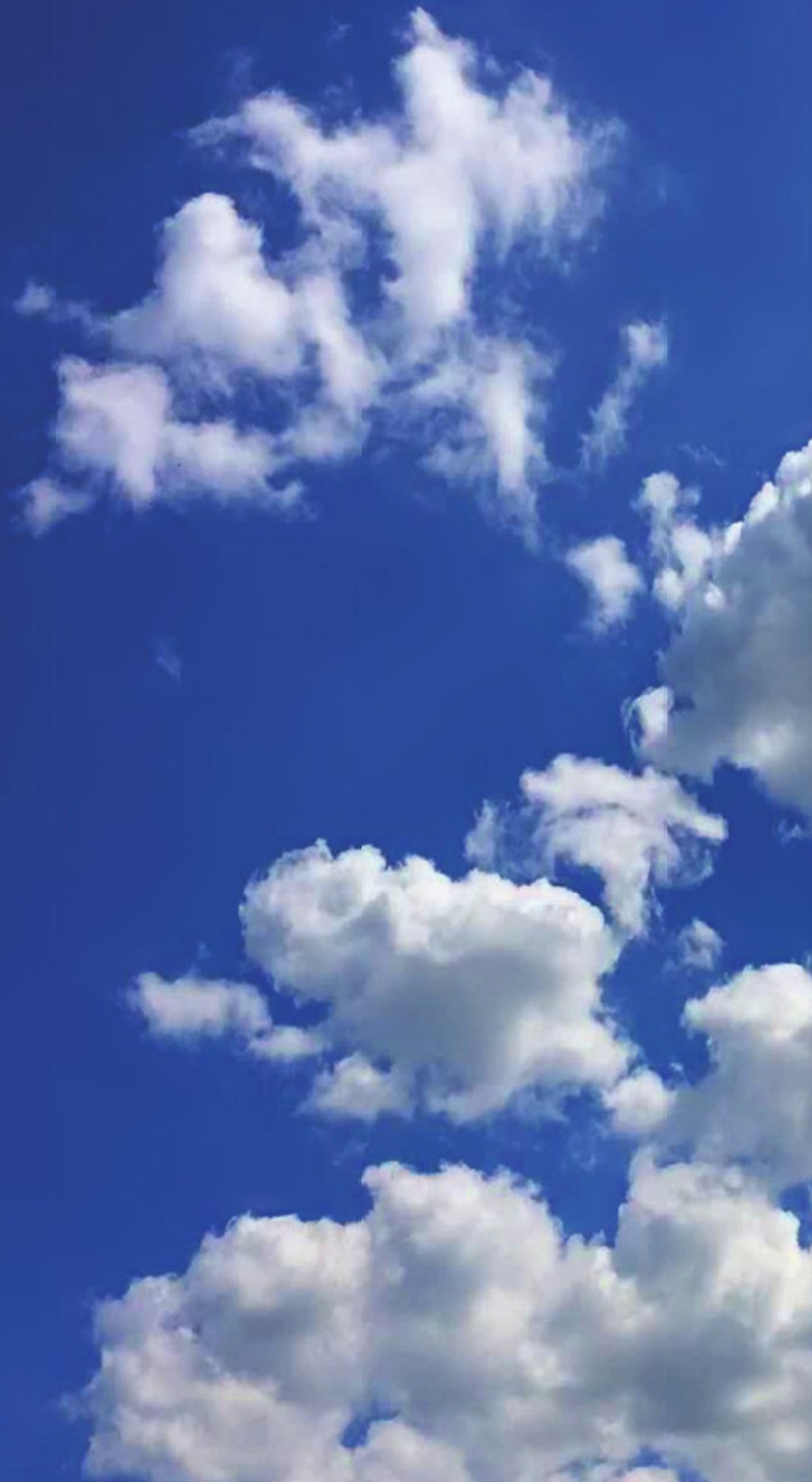




\section{Chapter 2}

The optimal approach to nutrition and cystic fibrosis: latest evidence and recommendations 


\section{ABSTRACT}

\section{Purpose of review}

Cystic fibrosis (CF) is a progressive genetic disease that affects multiple organ systems. Therapy is directed to maintain and optimize nutritional status and pulmonary function, as these are key factors in survival. In this review, the most recent findings regarding nutritional management associated with pulmonary function and outcome will be explored.

\section{Recent findings}

Evidence-based and expert-based guidelines emphasize the need for adequate nutritional intake to improve nutritional status. For infants and young children, the aim is to achieve the 50th percentile of weight and length for a healthy same-age population up to age 2 years. For older children and adolescents 2-18 years, the target is a body mass index (BMI) of at or above the 50th percentile for healthy children. For CF adults of at least 18 years, the target is a BMI of at or above $22 \mathrm{~kg} / \mathrm{m}^{2}$ for women and at or above $23 \mathrm{~kg} / \mathrm{m}^{2}$ for men. Recently, new drugs are developed with the aim to treat the malfunction of the cystic fibrosis transmembrane conductance regulator gene. This potentiator/corrector therapy improves lung function and nutritional status and decreases the number of infective exacerbations. As survival is improving and the CF population is aging, it is important to focus on micronutrient and macronutrient intake of CF patients in different age and disease stages.

\section{Summary}

Recent evidence-based nutritional guidelines and improved medical treatment support the nutritional monitoring and interventions in CF patients. Nutritional care should be personalized and provided by a specialized CF dietitian because patients' care needs may change dramatically during their disease progress. 


\section{INTRODUCTION}

Recent decades have shown a great improvement in survival of individuals with cystic fibrosis (CF). ${ }^{1}$ Registries in Europe, the USA and Canada now demonstrate that median survival for CF is around 40-50 years (UK 45.1, USA 41.1 and Canada 49.7 years). The median age at death worldwide is around 30 years ${ }^{1,2}$ because of health inequalities with the dramatic variation in the survival of CF individuals across Europe. ' The increased survival can be explained by implementation of centralized care, newborn screening programs (NBS) and evidencebased guidelines including better nutritional care and the introduction of drugs aimed at the modulation of cystic fibrosis transmembrane conductance regulator (CFTR) protein.

NBS programs for CF have been widely adopted in several European countries, Australia, New Zealand and the majority of states in North America. Major improvements in both nutritional status and lung function in young children, adolescents and adults as a result of NBS have been reported. Early diagnosis of CF by NBS and early intervention, including nutritional supplementation to prevent malnutrition, mucolytic therapy and aggressive treatment of infection with Pseudomonas aeruginosa, may have contributed to these improvements. ${ }^{3,4}$

Moreover, nutritional status is positively associated with pulmonary function and survival in patients with CF. This strongly supports the nutritional strategies and the efforts to obtain normal growth in CF children, and the importance to maintain adequate nutritional status in adults with CF. Careful nutritional monitoring as recommended in European Society of Parenteral and Enteral Nutrition (ESPEN)-ESPHAN-European Cystic Fibrosis Society (ECFS) guidelines ${ }^{5 . .}$ should continue to apply current early growth recommendation, with goal weight-for-length (WFL) at or above 50th percentile on growth charts before age $2 .{ }^{6 \cdots, 7}$ Timely and focused interventions to improve nutritional status are of great importance to improve prognosis and survival in CF.

The standard nutritional care for CF has been a high caloric diet with pancreatic enzyme replacement therapy (PERT) and fat-soluble vitamin supplementation to achieve an adequate nutritional status. ${ }^{5 \cdot-}$ The nutritional status can also be improved by using CFTR modulators, including correctors and potentiators. The effect of CFTR potentiation on clinical outcome and subsequently improved nutritional status is well described by Borowitz et al${ }^{8 *}$ (Table 1).

A CFTR potentiator, known as Ivacaftor and commercially available as Kalydeco, has produced results as respiratory function gain, and pulmonary exacerbations reductions, and several 
other achievements, such as better control of diabetes, improvement of pancreatic function, increase of body weight, improvement of BMI and BMI $z$ scores and quality of life. ${ }^{9-12,13,14}$ Other studies show an improvement in lung function, ${ }^{12}$ a slower rate of lung function decline ${ }^{10}$ or no significant changes. ${ }^{11}$ With more knowledge about genetic modifiers of the disease, the individual prognosis can be better defined and individualized therapy can be optimized.

Table 1 Potential mechanisms through which Ivacaftor might contribute to improved nutritional status

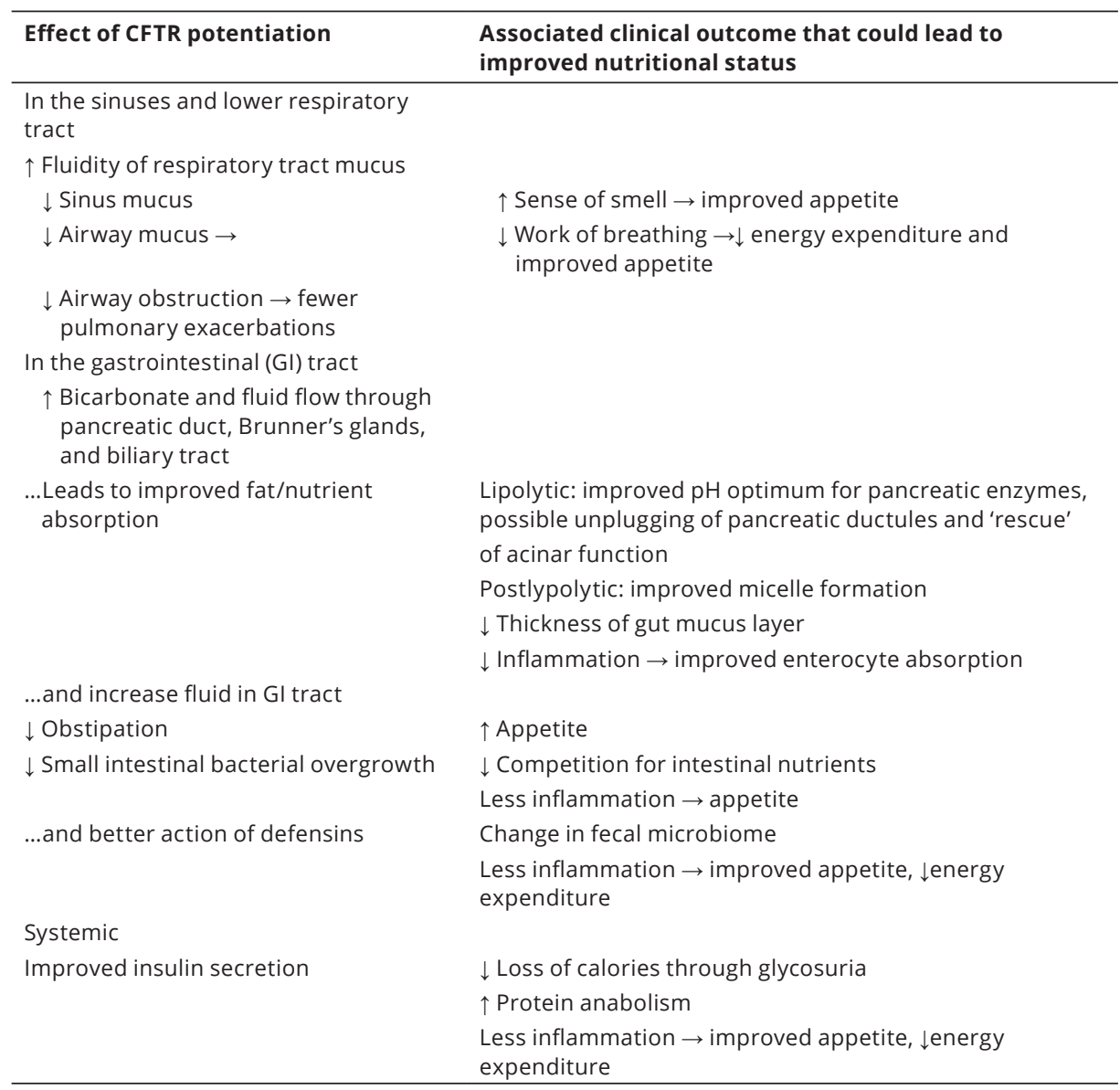

Reproduced from [8-・].

As survival has increased, the number of adults with CF is expanding, as the majority of children with CF now live into adulthood. Evidence-based nutritional guidelines for CF have 
been developed recently with more attention to adequate treatment of nutritional-related complications in CF such as CF-related diabetes (CFRD) and malnutrition. ${ }^{15,16}$

\section{NUTRITIONAL MANAGEMENTOF CYSTIC FIBROSIS; AN UPDATE FOR ADEQUATE TREATMENT ACROSS THE LIFE SPAN}

Recently, excellent evidence-informed and practice-based guidelines on nutrition care of infants, children and adults with CF have been developed by the ESPEN-European Society of Paediatric Gastroenterology, Hepatology and Nutrition (ESPGHAN)-ECFS, ${ }^{5 . \cdot}$ and also the Cystic Fibrosis Foundation (CFF) evidence-informed guidelines on enteral tube feeding (ETF). ${ }^{17}$ In addition, the Australian evidence-based guidelines are expected for October 2017....

Also (systematic) reviews on oral calorie supplements for CF, vitamin D, vitamin $E$, omega-3 poly unsaturated acids, probiotics, insulin and oral agents for managing CF-related diabetes endorse the importance of adequate nutritional treatment at different age- and disease stages in CF. $19-24,25 \cdots, 26-28$

\section{IMPORTANCE OF ADEQUATE GROWTH AND NUTRITIONAL ASSESSMENT}

$\mathrm{BMI}$ and BMI percentile for age are important measures of nutritional status in both adults and children with CF. The goal is a WFL of at or above the 50th percentile in children less than 2 years of age and a BMI of at or above the 50th percentile for children older than 2 years, meaning that nutritional status is comparable with that of well-nourished healthy children. ${ }^{5 \cdots, \cdots \cdots, 7}$ The rationale for this goal is that a positive association exists between pulmonary function, generally measured by forced expiratory volume in $1 \mathrm{~s}$ (FEV1) percentage predicted and nutritional status. ${ }^{6 *, 29}$ Children with CF who achieve higher WFL at age 2 years have improved pulmonary and survival outcomes into adulthood. CF care providers should be using growth charts appropriate to the nationality and ethnicity of the patients. If these are not available, the World Health Organization (WHO) growth charts should be used www.who. int/childgrowht/standards/en/.5. Growth and nutritional status should be monitored as part of routine CF care, including pubertal status. ${ }^{6 *}$ For CF adults over the age of 18 years, the target is a BMl of at or above $22 \mathrm{~kg} / \mathrm{m}^{2}$ for women and $23 \mathrm{~kg} / \mathrm{m}^{2}$ for men. ${ }^{5 \cdots, 30 .}$ Methods and timing of 
assessment and monitoring of nutritional status in age-related CF people are well described in the ESPEN-ESPGHAN-ECFS guidelines. ${ }^{5 . *}$ In adults, assessment of body composition is becoming clinically important as depleted or low fat free mass (FFM) is associated with significant lung disease and impaired pulmonary function ${ }^{15}$ and independent of BMI level. ${ }^{30}$. In children, bone mineral content (BMC) is a more sensitive indicator of nutritional deficit than low BMI; low values of BMC are correlated with impaired pulmonary function in children with CF. ${ }^{5 . *}$ Furthermore, total body potassium counting is a body composition assessment method that measures the body cell mass (BCM). The BCM is the metabolically active component of the FFM and reflects the functional cellular components of the body involved in biochemical processes and energy metabolism. BCM is not influenced by hydration. In contrast to the interpretation of total FFM, which can be affected by hydration changes with growth and disease. ${ }^{31}$ Therefore, BCM measurements are an important reflection of nutritional status in growing children and those with clinical conditions. However, this method may not be widely available and other methods such as bioelectrical impedance analysis and dual energy X-ray absorptiometry can provide information on fat mass and FFM and should be used where possible. Future research needs to focus on perfecting bedside techniques to assess body composition, which will assist in improving nutrition-related outcome measures. ${ }^{31}$

\section{ENERGY BALANCE IN CYSTIC FIBROSIS}

Optimal energy intake is critical to the overall health of people with CF. Insufficient nutritional intake is common in the CF population and is caused by poor appetite, malabsorption and disturbed body image. This, in combination with increased caloric expenditure, makes it often difficult to attain an appropriate nutritional status. ${ }^{6 \cdot *}$

The energy balance is not only determined by energy intake (food), energy expenditure (activity, maintenance and increased demands with inflammation) and losses (diarrhea and vomiting). In CF, a variety of factors contribute to individual energy needs, including nutrient maldigestion and/or malabsorption, presence of pulmonary exacerbation, pulmonary function, FFM, sex, pubertal status, CFTR mutation, age and other medical complications, such as liver disease and CFRD. ${ }^{6 \cdot *}$

Loss of energy due to malabsorption is a problem in CF patients with exocrine pancreatic insufficiency. PERT is essential to improve fat malabsorption in pancreatic insufficient patients. ${ }^{32}$ Eighty-five percent to $90 \%$ of people with CF have pancreatic insufficiency, leading 
to malabsorption of nutrients, especially fat and fat-soluble vitamins ${ }^{6 \cdots, 32 .}$ Addition of proton pump inhibitors may improve effectiveness of PERT. ${ }^{32}$. Current guidelines recommend lipase intake by age of the patient, by body weight and by grams of fat ingested per day, ${ }^{5 . *}$ but a large variability and inconsistency with new guidelines on nutrition and PERT-use was found in six European pediatric CF centers. ${ }^{33}$ The MyCyFAPP Project has been started to develop educational and self-management tools for patients' better adherence to therapies. ${ }^{33}$

Increased caloric expenditure is mainly caused by increased work of breathing due to chronic lung infection and loss of pulmonary function. In addition, chronic pulmonary infection and inflammation lead to cytokine-induced catabolism. ${ }^{6 *}$ In patients with end-stage $C F$, predication equations for energy needs underestimate resting energy expenditure (REE). ${ }^{34}$

There is a wide variation in the energy requirements of people with CF. To achieve a normal growth and nutritional status, energy intake targets for age may need to be increased in children with $\mathrm{CF}$, although obesity should be avoided. 5." For this reason, recommendations for energy needs are $110-200 \%$ of those required by the healthy population of the sex and age $5 ., 15$. Improvement in weight gain can be achieved using energy dense diets, and additional oral nutritional supplements (ONS), behavioral interventions, ETF or parenteral nutrition. ${ }^{5 . \cdot, 15}$

A recent systematic review of ONS in three randomized clinical trials (total of 131 patients) found that ONS did not promote additional weight gain in moderately malnourished children with CF over and above the use of dietary advice and monitoring alone. ${ }^{19}$ In contrast with children, the use of ONS in adults with CF has not been adequately studied. ${ }^{5}$ Although the Cochrane review concluded that there is not enough evidence to support the use of ONS, practice-based evidence has shown that in clinical practice, individually prescribed supplements have increased energy intake and weight in undernourished patients, ${ }^{5 . *}$ but further research should investigate the long-term effect of supplement use.

The CFF recommends use of ETF in people with CF who are unable to attain caloric requirements to meet growth/weight maintenance goals despite evaluation by a multidisciplinary team. ${ }^{17}$ Using this intervention, it is important to monitor for complications such as glucose intolerance and glucosuria. Gastrostomy tubes are commonly used in patients using ETF, and jejunostomy tubes are placed in patients with gastro paresis. ${ }^{30 .}$

Although CF is commonly associated with malnutrition, the proportion of overweight and obese individuals is increasing. In one US pediatric CF center, $23 \%$ of patients with CF aged 
2-18 years were found to have an average BMI percentile greater than 90 . Surprisingly, $88 \%$ of the overweight patients and $69 \%$ of the obese patients had CFTR mutations associated with pancreatic insufficiency. In a longitudinal cohort study that spanned from 1985 to 2011, the proportion of overweight or obese adults in a Canadian CF center increased from $7 \%$ to $18 \%$. The benefit of increasing BMI greater than 25 in adults seems to be small as improvement in pulmonary function seems to be blunted. ${ }^{30}$. Moreover, recent studies show a higher proportion of obese individuals with CF having elevated serum triglycerides and total cholesterol. ${ }^{30}$. Furthermore, a cross sectional study in 32 adults with CF and a reference group of 20 adults without CF showed that a normal-weight BMI with elevated percentage body fat is associated with reduced lung function. ${ }^{35 .}$ Dietary recommendations should therefore focus on a balanced diet and a healthy lifestyle with good exercise habits to achieve an adequate body composition with normal fat and FFM percentages.

\section{SPECIALIZED NUTRITION-RELATED TREATMENTS IN CYSTIC FIBROSIS}

For CF patients with pancreatic insufficiency, recent guidelines recommend the evaluation of plasma levels of fat-soluble vitamins after the start of enzyme and vitamin supplementation 3-6 months after initiation or change in vitamin therapy; and annually thereafter. ${ }^{5 \cdot .} \mathrm{A}$ review of Li et al. ${ }^{36}$ compared recent reports of actual dietary intake and nutritional status of micronutrients such as minerals, trace elements and vitamins with relevant dietary recommendations for CF. They conclude that although dietary intake and nutritional status in CF have improved significantly in recent decades, micronutrient status seems to have diverged. Recommendations for different age groups differ between countries. ${ }^{36}$ The optimal dosages of long-term micronutrient supplementation require further investigation, so that safety and effect on reducing lung disease severity and CF-related complications are balanced. ${ }^{20,36}$ Vitamin $\mathrm{D}$ is under investigation for its potential role in gut microbiota modification, ${ }^{37}$ intestinal calcium absorption and bone health, ${ }^{5 \cdots, 22}$ recovery from pulmonary exacerbations ${ }^{22}$ and improvement of lung function. ${ }^{23}$ The positive results observed in CF and non-CF trials of vitamin D supplementation provide a strong rationale for larger, randomized control trials of long-term, high-dose vitamin D3 supplementation in CF patients. ${ }^{23}$

CF patients usually have abnormal intestinal microbiota because of exposure to antibiotics. Probiotics could modify the gut microbiota. Several reviews examined the use of probiotics in the treatment of CF pulmonary exacerbation and intestinal inflammation. $25 . \cdot 26,28,38$ 
Unfortunately, they lack the scientific quality that is needed for a recommendation. Until there is more robust evidence supporting the safety of probiotics in clinical care, they should be used with caution with high-risk patients such as those with acute exacerbations or those with severe respiratory function. ${ }^{25 . *}$

Also the evidence for dietary supplementation of fatty acids to improve lung function or anti-inflammatory effects ${ }^{39}$ in children or adults with CF is too limited for recommendations in daily practice. ${ }^{5 \cdots, 21}$

Treatment of CFRD includes education on diabetes self-management, insulin therapy and aerobic exercise. Although some CF centers use oral medications to help control diabetes, the CFF clinical practice guidelines support the use of insulin therapy and this remains the most widely used treatment method. ${ }^{24}$ The achievement of adequate blood glucose levels would be best to prevent decline in pulmonary function. ${ }^{27}$ Patients need to learn to adjust their insulin dose to the carbohydrate content of the meal, sip feeds or ETF. CFRD patients can benefit from being seen periodically by a specialized team with expertise in both diabetes and CF. ${ }^{5 \cdot}$

For CF women planning to become pregnant, a daily supplement of $400 \mu \mathrm{g}$ of folic acid in the preconception period and throughout the first trimester to prevent neural tube defects is recommend, ${ }^{\text {,** }}$ similar to non-CF women. A variety of complications may occur in pregnant CF women, including impaired airway clearance, chronic respiratory tract infections, diabetes mellitus, pancreatic insufficiency and nutritional deficiencies. Pregnancy is advised against if the FEV1 is less than $30 \%$ predicted or if pulmonary hypertension is present. ${ }^{40}$ Pregnant women with moderate pulmonary function increase the probability of an optimal outcome. ${ }^{41}$ Management includes adequate nutrition with PERT and fat-soluble vitamins, management of the chest infections with antibiotics and monitoring of maternal diabetes..$^{40}$ The third trimester may be associated with increasing dyspnea that may necessitate bed rest with supplemental oxygen and nutritional supplements. ${ }^{41}$

Lung transplantation (LTX) is a well-established treatment option for CF patients with endstage lung disease. Ongoing weight loss despite aggressive nutritional supplementation is one of the criteria for listing for LTx. ${ }^{42}$ It is a challenge for dietitians to optimize energy requirements for improving the nutritional status in pre-LTx patients with CF. Measurement of REE can be a helpful tool to optimize nutritional intervention as prediction equations seem to underestimate REE in end-stage CF patients. ${ }^{34}$ After LTX, patients with CF are at risk of 
developing diabetes, and both tacrolimus and systemic steroids are known to increase the risk of diabetes posttransplant. ${ }^{43}$

\section{CONCLUSION}

Nutritional care should be closely adapted to the various stages and complications of CF across the lifespan to extend survival and to improve quality of life. Moreover, new therapies, such as CFTR modulators and transplantation techniques, change the needs for nutritional intervention. Recent evidence-based and expert-based guidelines provide up-to-date information for optimal nutritional care of the general patient, but for treatment of individual CF patients personalized treatment by a specialized CF dietitian is recommended.

Financial support and sponsorship There is no funding to disclose.

Conflicts of interest There are no conflicts of interest. 


\section{REFERENCES AND RECOMMENDED READING}

Papers of particular interest, published within the annual period of review, have been highlighted as:

- of special interest

.. of outstanding interest

1. Elborn JS, Bell SC, Madge SL, et al. Report of the European Respiratory Society/European Cystic Fibrosis Society task force on the care of adults with cystic fibrosis. Eur Respir J 2016;47:420-428.

2. Salvatore D, Buzzetti R, Mastella G. Update of literature from cystic fibrosis registries 2012-2015. Part 6: epidemiology, nutrition and complications. Pediatr Pulmonol 2017;52:390-398.

3. Mak DY, Sykes J, Stephenson AL, Lands LC. The benefits of newborn screening for cystic fibrosis: the Canadian experience. J Cyst Fibros 2016;15:302-308.

4. Leung DH, Heltshe SL, Borowitz D, et al. Effects of diagnosis by newborn screening for cystic fibrosis on weight and length in the first year of life. JAMA Pediatr 2017;171:546-554.

5.-. Turck D, Braegger CP, Colombo C, et al. ESPEN-ESPGHAN-ECFS guidelines on nutrition care for infants, children, and adults with cystic fibrosis. Clin Nutr 2016;35:557-577.

Up-to-date evidence-based nutritional care guideline developed by a team of experts representing ESPEN, ESPGHAN and the ECFS who systematically reviewed the literature to summarize current knowledge on epidemiology and pathophysiology, prevention, and treatment of CF-related undernutrition.

6.- $\quad$ Lusman S, Sullivan J. Nutrition and growth in cystic fibrosis. Pediatr Clin North Am 2016;63:661-678. An important review about nutrition and growth in CF emphazising the need that CF children achieve growth and nutritional status comparable with that of well-nourished non-CF children. Measurements of antropometrics such as weight, lengte of height (including the midparental target height prediction), $B M I$ percentile and pubertal status should be monitored as part of routine CF care.

7. Usatin D, Yen EH, McDonald C, et al. Differences between WHO AND CDC early growth measurements in the assessment of cystic fibrosis clinical outcomes.J Cyst Fibros 2017;16:503-509.

8.-- $\quad$ Borowitz D, Lubarsky B, Wilschanski M, et al. Nutritional status improved in cystic fibrosis patients with the G551D mutation after treatment with Ivacaftor. Dig Dis Sci 2016;61:198-207.

First study demonstrating the improvement of nutritional status across a range of children, adolescents, and adults with CF and the most common CF gating mutations with Ivacaftor treatment. Futhermore, including an interesting table with potential mechanisms though which Ivacaftor might contribute to improved nutritional status.

9. Michl RK, Tabori H, Hentschel J, et al. Clinical approach to the diagnosis and treatment of cystic fibrosis and CFTR-related disorders. Expert Rev Respir Med 2016:1-10.

10. Konstan MW, McKone EF, Moss RB, et al. Assessment of safety and efficacy of long-term treatment with combination lumacaftor and Ivacaftor therapy in patients with cystic fibrosis homozygous for the F508del-CFTR mutation (PROGRESS): a phase 3, extension study. Lancet Respir Med 2017;5:107118.

11. Milla CE, Ratjen F, Marigowda G, Liu F, et al. Lumacaftor/lvacaftor in patients aged 6-11 years with cystic fibrosis and homozygous for F508del-CFTR. Am J Respir Crit Care Med 2017;195:912-920. 
12. Dryden C, Wilkinson J, Young D, Brooker RJ; Scottish Paediatric Cystic Fibrosis Managed Clinical $\mathrm{N}$. The impact of 12 months treatment with Ivacaftor on Scottish paediatric patients with cystic fibrosis with the G551D mutation: a review. Arch Dis Child 2016; pii:archdischild-2015-310420. doi:10.1136/archdischild-2015-310420.

13.- McColley SA. A safety evaluation of Ivacaftor for the treatment of cystic fibrosis. Expert Opin Drug Saf 2016;15:709-715.

An interesting review that focuses on the safety of Ivacaftor which is indicated for treatment of CF mediated by 10 mutations of the CFTR gene that causes gating or partial function abnormalities. Clear overview to read on mechanism of action, metabolism and clinical application, including safety evaluation and expert opinion.

14. Castellani C, Assael BM. Cystic fibrosis: a clinical view. Cell Mol Life Sci 2017;74:129-140.

15. Collins S. Nutritional management of cystic fibrosis an update for the 21st century. Paediatr Respir Rev 2017. pii:S1526-0542(17)30029-5. doi:10.1016/j.prrv.2017.03.006.

16. Wolfe SP, Wilschanski M. Nutrition in CF: Two new important guidelines. J Cyst Fibros 2016;15:708709.

17. Schwarzenberg SJ, Hempstead SE, McDonald CM, et al. Enteral tube feeding for individuals with cystic fibrosis: Cystic Fibrosis Foundation evidence-informed guidelines. J Cyst Fibros 2016;15:724735 .

18.-- Saxby N, Painter C, Kench A, et al., and the Australian and New Zealand Cystic Fibrosis Nutrition Guideline Authorship Group. Nutrition guidelines for cystic fibrosis in Australia and New Zealand. Scott CB, editor. Sydney: Thoracic Society of Australia and New Zealand; 2017.

The most up-to-date evidence-based nutrition guidelines and consensus recommendations for cystic fibrosis are developed in Australia and New Zealand and final version is expected October 2017. This is performed by an expert group using PICO ( $P$ - patient, problem or population; I - intervention; $C$ comparison, controle or comparator; O - outcome) method.

19. Smyth RL, Rayner O. Oral calorie supplements for cystic fibrosis. Cochrane Database Syst Rev 2017;5:CD000406.

20. Okebukola PO, Kansra S, Barrett J. Vitamin E supplementation in people with cystic fibrosis. Cochrane Database Syst Rev 2017;3:CD009422.

21. Oliver C, Watson H. Omega-3 fatty acids for cystic fibrosis. Cochrane Database Syst Rev 2016(1):CD002201. doi: 10.1002/14651858.CD002201.pub5.

22. Chesdachai S, Tangpricha V. Treatment of vitamin D deficiency in cystic fibrosis. J Steroid Biochem Mol Biol 2016;164:36-39.

23. Konstantinopoulou S, Tapia IE. Vitamin D and the lung. Paediatr Respir Rev 2016.pii:S15260542(16)30123-3. doi: 10.1016/j.prrv.2016.10.009.

24. Onady GM, Stolfi A. Insulin and oral agents for managing cystic fibrosis-related diabetes. Cochrane Database Syst Rev 2016;4:CD004730.

25.-- Anderson JL, Miles C, Tierney AC. Effect of probiotics on respiratory, gastrointestinal and nutritional outcomes in patients with cystic fibrosis: a systematic review. J Cyst Fibros 2017;16:186-197. 
This systematic review shows that there is insufficient high-quality evidence at this time to recommend a specific probiotic species and strain, dose or frequency, as being the most promising for improving health outcomes such as reducing pulmonary exacerbations and decreasing gastrointestinal inflammation in children and adults with CF. Further research is recommended to assist clinicians in making the best recommendations for their CF patients. The latter corresponds to Van Biervliet's study (see also reference [38]).

26. Ananthan $\mathrm{A}$, Balasubramanian $\mathrm{H}$, Rao $\mathrm{S}$, Patole $\mathrm{S}$. Probiotic supplementation in children with cystic fibrosis: a systematic review. EurJ Pediatr 2016;175:1255-1266.

27. Jones GC, Sainsbury CA. A practical approach to glucose abnormalities in cystic fibrosis. Diabetes Ther 2016;7:611-620.

28. Nikniaz Z, Nikniaz L, Bilan N, et al. Does probiotic supplementation affect pulmonary exacerbation and intestinal inflammation in cystic fibrosis: a systematic review of randomized clinical trials. World J Pediatr 2017. doi:10.1007/s12519-017-0033-6.

29. Lahiri T, Hempstead SE, Brady C, et al. Clinical practice guidelines from the cystic fibrosis foundation for preschoolers with cystic fibrosis. Pediatrics 2016;137:1-26.

30.- Solomon M, Bozic M, Mascarenhas MR. Nutritional issues in cystic fibrosis. Clin Chest Med 2016;37:97-107.

This interesting article focuses on nutritional issues in children, adolescents and adults with CF. Discussed topics are the risk for malnutrition, CF-related complications, comprehensive nutritional assessments and nutritional support to ensure optimal growth and nutritonal status.

31. Murphy AJ, Hill RJ, Buntain $\mathrm{H}$, White $\mathrm{M}$, et al. Nutritional status of children with clinical conditions. Clin Nutr 2017;36(3):788-92.

32." Working Party of the Australasian Pancreatic C. Smith RC, Smith SF, Wilson J, et al. Summary and recommendations from the Australasian guidelines for the management of pancreatic exocrine insufficiency. Pancreatology 2016;16(2):164-180.

This review article summarized the literature and created new guidelines for management of pancreatic exocrine insufficiency. Recommendations for the use of PERT and optimal monitoring in patients with CF are clearly stated.

33. Calvo-Lerma J, Hulst JM, Asseiceira I, et al. Nutritional status, nutrient intake and use of enzyme supplements in paediatric patients with Cystic Fibrosis; a European multicentre study with reference to current guidelines. J Cyst Fibros 2017;16:510-518.

34. Hollander FM, Kok A, de Roos NM, et al. Prediction equations underestimate resting energy expenditure in patients with end-stage cystic fibrosis. Nutr Clin Pract 2017;32:116-121.

35.- Alvarez JA, Ziegler TR, Millson EC, Stecenko AA. Body composition and lung function in cystic fibrosis and their association with adiposity and normal-weight obesity. Nutrition 2016; 32:447452.

This is the first pilot study demonstrating an independent, inverse relationship between measure of adiposity and lung function in individuals with CF. Prospective studies are needed to explore the longterm health implications in CF and may require re-evaluation of current nutritional monitoring and interventions in CF individuals.

36. Li L, Somerset S. Dietary intake and nutritional status of micronutrients in adults with cystic fibrosis in relation to current recommendations. Clin Nutr 2016;35:775-782. 
37. Kanhere M, Chassaing B, Gewirtz AT, Tangpricha V. Role of vitamin D on gut microbiota in cystic fibrosis. J Steroid Biochem Mol Biol 2016. pii:S0960-0760(16)30296-5. doi:10.1016/j. jsbmb.2016.11.001.

38. Van Biervliet S, Declerqc D, Somerset S. Clinical effects of probiotics in cystic fibrosis patients: a systematic review. Clinical Nutrition ESPEN 2017;18:37-43.

39. Husson MO, Ley D, Portal C, et al. Modulation of host defence against bacterial and viral infections by omega-3 polyunsaturated fatty acids. J Infect 2016;73:523-535.

40. Annamraju H, Mackillop L. Respiratory disease in pregnancy. Obstet Gynaecol Reprod Med 2017;27:105-111.

41. Leidecker K, Dorman K. Pulmonary Disorders Complicating Pregnancy: An Overview. J Perinat Neonatal Nurs 2016;30:45-53.

42. Morrell MR, Pilewski JM. Lung transplantation for cystic fibrosis. Clin Chest Med 2016;37:127-138.

43. Faro A, Weymann A. Transplantation. Pediatr Clin North Am 2016;63:709-734. 
The optimal approach to nutrition and cystic fibrosis 
蛋

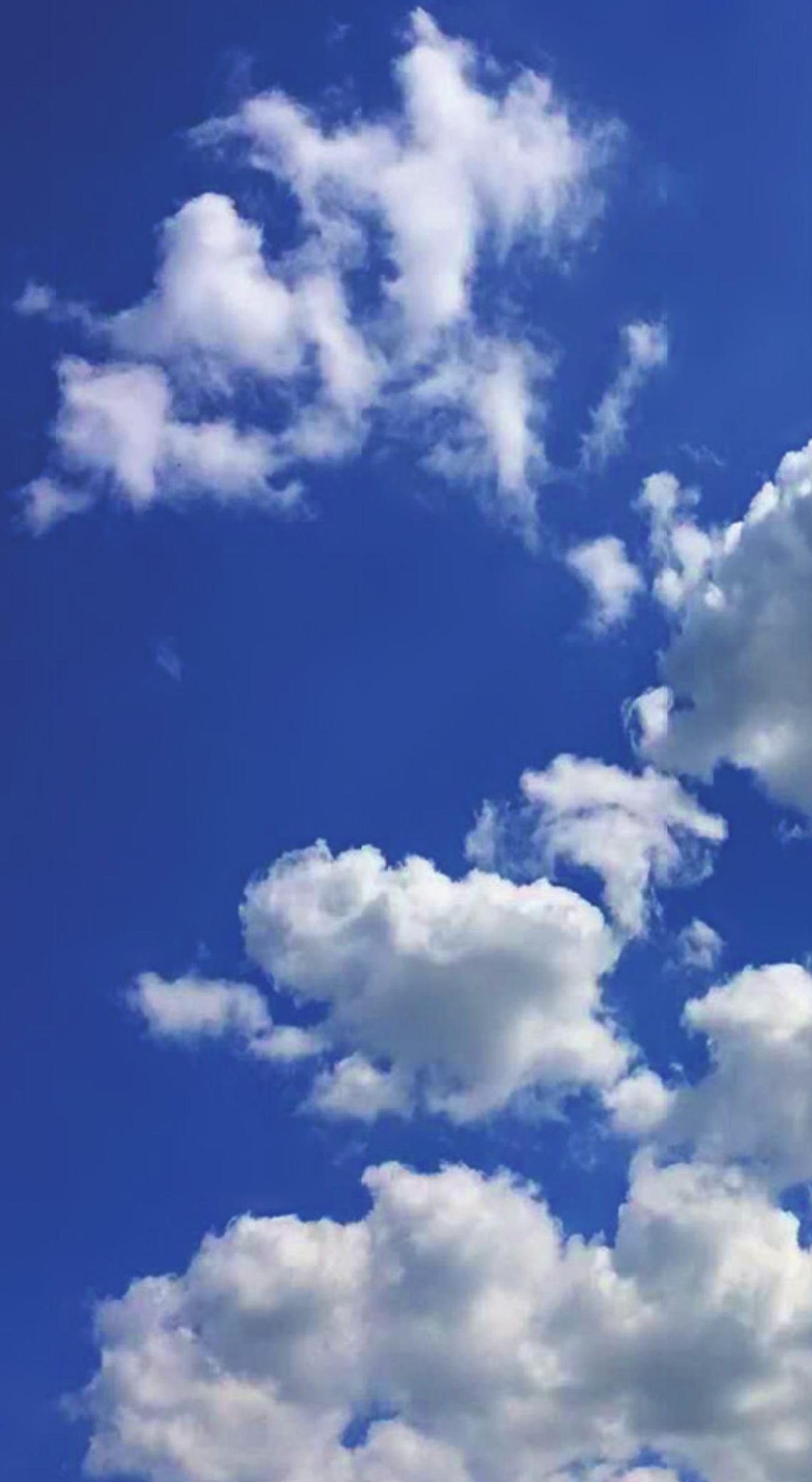




\section{Chapter 3}

\section{Prediction equations underestimate resting energy expenditure in patients with end-stage cystic fibrosis}

Francis M. Hollander

Annemieke Kok

Nicole M. de Roos

Gerdien Belle-van Meerkerk

Ed A. van de Graaf

Nutrition in Clinical Practice 2017 Feb;32(1):116-121 


\section{ABSTRACT}

\section{Background}

Resting energy expenditure (REE) is increased in patients with cystic fibrosis (CF) with end-stage lung disease due to chronic inflammation and pulmonary infections. After lung transplantation (LTX), energy expenditure is expected to be lower because inflammation will decrease. We assessed the agreement between measured and predicted REE in pre-LTX CF and post-LTx patients with CF and differences in REE in pre-LTX CF and post-LTx patients with CF in a cross-sectional study.

\section{Methods}

Included were 12 pre-LTx patients with CF (9 women; median age 31.6 years; interquartile range [IQR], 23.3-40.0) and 12 patients with CF within 2 years after LTx (6 women; median age 33.5 years; IQR, 22.3-40.3). REE was measured in a fasted state using indirect calorimetry. Values were compared with predicted REE calculated by formulas of Harris Benedict (1919 and 1984), Schofield, and the World Health Organization (1985). A calculated REE between $90 \%$ and $110 \%$ of REE measured was considered adequate.

\section{Results}

Prediction equations underestimate REE in at least $75 \%$ of pre-LTX and 33\% of post-LTX patients with CF. Mean (SD) REE measured by indirect calorimetry was 1735 (251) kcal pre-LTx and $1650(235) \mathrm{kcal}$ ) post-LTx $(P=.40)$. REE expressed per kilogram of fat-free mass (FFM) was $40.5 \mathrm{kcal} / \mathrm{kg}$ in pre-LTx patients with CF, which was higher than the $34.3 \mathrm{kcal} / \mathrm{kg}$ in post-LTX patients with CF patients $(P=.01)$.

\section{Conclusions}

Prediction equations underestimate REE in patients with end-stage CF. REE per kg of FFM is lower post-LTX than pre-LTX in patients with CF. Measurement of REE is recommended for patients with CF, especially pre-LTX, to optimize energy requirements for improving nutritional status. 


\section{INTRODUCTION}

Nutritional status has a strong positive association with pulmonary function and survival in cystic fibrosis (CF). ${ }^{1}$ Nutrition guidelines for patients with CF recommend energy intakes of $110 \%-200 \%$ of requirements for the healthy population of similar age, sex, and size. ${ }^{2}$ Most patients with CF require a higher energy intake than healthy individuals due to chronic inflammation and pulmonary infections. ${ }^{3}$ In addition, a reduction in dietary intake, especially during episodes of infection, and increased energy losses from fat malabsorption have a negative impact on nutritional status. ${ }^{3,4}$ Insights into energy requirements are necessary for determining dietary requirements to achieve an adequate nutrition status.

In general, to determine energy requirements in patients, different prediction equations can be used in which resting energy expenditure (REE) is multiplied by activity and disease factors. ${ }^{5,6}$ Therefore, REE is an important determinant of total energy expenditure (TEE). REE can be calculated based on age, sex, and body weight in healthy persons, using raw data of prediction equations such as those of Harris-Benedict (HB; 1919 and 1984), ${ }^{6}$ Schofield, ${ }^{5}$ and the World Health Organization (WHO; 1985). ${ }^{7}$

For patients with $\mathrm{CF}$, there is no consensus on which prediction equation is most reliable. The prediction equations of the WHO and HB (1919) underestimate REE in adult patients with CF with exocrine pancreatic insufficiency (PI) and homozygote $\Delta \mathrm{F} 508$ compared with measured REE by indirect calorimetry. ${ }^{8}$ Moreover, commonly used prediction equations are often not suitable for calculating REE in critically ill or hospitalized patients. ${ }^{9}$ Different prediction equations can overestimate or underestimate REE in adults with CF. ${ }^{10}$ This will provide inadequate individual recommendations for TEE and impairs reaching an optimal nutrition status.

Previous studies have shown that nutrition status predicts survival and affects outcome in adult patients with CF pre- and post-LTX.11,12 In our own LTx patients with CF, a low body mass index (BMI) and especially a low fat-free mass index (FFMI) at waiting list entry impaired survival. ${ }^{11}$ Despite dietary treatment and tailored exercise training, no significant improvement in BMI or FFMI was seen between waiting list entry and the last outpatient visit pre-LTx. This might be due to underestimated energy requirements used in the dietary advice. ${ }^{11}$ These results suggest that adequate prediction of REE by equations is hampered by the increased energy requirements due to lung disease severity. Correct REE values will improve estimation of TEE, and this will provide adequate individual recommendations for energy. 
Indirect calorimetry is the most commonly used and noninvasive method for measuring REE in clinical populations, as well in CF. ${ }^{13,14}$ We found no studies that compared the validity of prediction equations for energy requirements pre-LTX and post-LTX with measured values. The primary aim of this study was to assess the agreement between measured REE using indirect calorimetry and predicted REE according to Harris-Benedict 1919 and 1984, Schofield, and WHO 1985 prediction equations. Our secondary aim was to assess differences in REE in pre-LTx CF and post-LTx patients with CF in a cross-sectional study.

\section{METHODS}

\section{Study Population and Characteristics}

From September 2014 through December 2014, all patients with CF who visited our outpatient clinic for lung transplantation at the University Medical Center Utrecht and who were either pre-LTX or within 2 years post-LTX were included for REE measurements. Unfortunately, no follow-up data on the same patients pre-LTX and post-LTx were yet available. Exclusion criteria for REE measurement were claustrophobia, pneumothorax, and intravenous antibiotic treatment. Data on sex, genotype mutation, exocrine PI, presence of cystic fibrosis-related diabetes (CFRD) or new-onset diabetes after transplant (NODAT), age, colonization with Pseudomonas aeruginosa, and pulmonary function were collected from electronic medical patient records. Chronic infection with $P$ aeruginosa was defined according to Lee et al. ${ }^{15}$ Pulmonary function was routinely assessed by spirometry (ZAN, Oberthulba, Germany) and expressed as $\mathrm{FEV}_{1} \%$ of the predicted normal forced expiratory volume in 1 second $\left(\mathrm{FEV}_{1}\right)$. All $\mathrm{FEV}_{1}$ measurements were performed by the Laboratory for lung function of the University Medical Center Utrecht prior to REE measurement. The study was approved by the Medical Ethics Committee of University Medical Center Utrecht.

\section{Anthropometric Data}

Measurements of body weight $( \pm 0.1 \mathrm{~kg})$ and height $( \pm 0.1 \mathrm{~cm})$ were performed in the Nutritional Assessment laboratory of the Department of Dietetics, using a calibrated electronic scale (SECA model 701; SECA, Hamburg, Germany) and stadiometer (SECA model 225). Patients were measured in fasted state dressed in indoor clothing and without shoes. BMI was classified according to the WHO recommendations: underweight, $<18,5 \mathrm{~kg} / \mathrm{m}^{2}$; normal weight, 18.5 $24.9 \mathrm{~kg} / \mathrm{m}^{2}$; and overweight, $25-29.9 \mathrm{~kg} / \mathrm{m}^{2}{ }^{2}{ }^{16}$ Fat-free Mass (FFM) was routinely measured in pre-LTx and post-LTx patients with CF at the Department of Lung Function using bioelectrical impedance analysis (BIA) (Body stat 1500; Bodystat Ltd, Douglas, UK). Patients were measured 
in fasted state and lying in supine position. A low FFMI was defined as $\leq 14.6 \mathrm{~kg} / \mathrm{m}^{2}$ for women and $\leq 16.7$ for men. ${ }^{17}$

\section{Measurement of REE}

REE was measured using indirect calorimetry by a ventilated hood system (COSMED Quark RMR; COSMED, Rome, Italy), which was calibrated daily before use. Measurements of REE were performed according to institutional standard operating procedures (SOP). A new disposable antibacterial filter was used in every REE measurement to prevent bacterial and viral crosscontamination. Patients were measured in a fasted and rested state of at least 8 hours. If patients were using oxygen supplementation, this was stopped before the REE measurement. Patients were in supine position. Measurements were performed in standard neutral hospital room temperature. A clear canopy was placed over the head of the patient. The canopy was disinfected with antiviral and antibacterial disinfection wipes after every REE measurement. Respiratory gas exchanges were monitored for 30 minutes. Data of steady-state ventilation of at least 15 to 20 minutes were achieved in all patients, when the average minute $\mathrm{VO}_{2}$ and $\mathrm{VCO}_{2}$ changes by $<10 \%$ and the average respiratory quotient changes by $<5 \%$. The Weir equation was used to calculate REE. ${ }^{18}$

\section{Calculation of REE by Prediction Equations}

Different prediction equations are used in clinical practice to calculate REE in patients with CF. According to an earlier study of validation of predictive equations for REE in adult outclinic and clinic patients, the most practical and relatively accurate equations were selected to compare with measured REE. ${ }^{19}$ The original Harris-Benedict (HB 1919) and revised HarrisBenedict equation by Roza and Shizgal (HB 1984), Schofield equation, and WHO prediction equation were used without activity and disease factors.

\section{Statistical/Data Analysis}

Normality was tested by histograms, Q-Q plots, and the Shapiro-Wilk test. Descriptive statistics were used to summarize baseline characteristics. Results are expressed as median \pm interquartile range (IQR) unless stated otherwise. Bland-Altman analysis was performed to examine agreement between measured vs predicted REE. Significant differences between groups were tested by the Mann-Whitney $U$ test for continuous variables and with the Fisher exact test for nominal data.

A calculated REE between $90 \%$ and $110 \%$ of REE measured was considered as an acceptable prediction; below $90 \%$ was considered an underestimation and above $110 \%$ an 
overestimation. ${ }^{20}$ Statistical analysis was performed using SPSS version 21.0 (SPSS, Inc, an IBM Company, Chicago, IL). A $P$ value of $<.05$ was considered to indicate statistical significance.

\section{RESULTS}

\section{Study Population}

Twenty-eight patients with CF were eligible for REE measurement. Four patients were excluded due to claustrophobia $(n=1)$, pneumothorax $(n=2)$, and death $(n=1)$ shortly before scheduled measurement. Twenty-four patients with CF were included, of whom 12 were pre-LTx and 12 were post-LTx (7 patients within 6-12 months post-LTx and 5 patients within 12-24 months post-LTX). Characteristics are shown in Table 1.

\section{Anthropometrics}

No significant differences between anthropometric measurements were seen pre-LTX and post-LTx. Underweight based on BMI $<18.5 \mathrm{~kg} / \mathrm{m}^{2}$ was seen in only 1 man in the pre-LTx group and 1 woman in the post-LTx group. Median FFMI was $15.0 \mathrm{~kg} / \mathrm{m}^{2}(14.4-16.0)$ in the group of patients pre-LTX and $16.8 \mathrm{~kg} / \mathrm{m}^{2}$ (14.8-17.6) in the group post-LTX. Four patients ( 3 women) had a low FFMI in the pre-LTx group and 1 woman within 2 years post-LTx group according to cutoff points by Kyle et al ${ }^{17}$ (data not shown).

\section{REE Measurement and Calculation by Prediction Equations}

Mean (SD) measured REE was 1735 (251) kcal in the patients who were measured pre-LTx and $1650(235) \mathrm{kcal}$ in the patients measured post-LTx (Table 2). This difference was not significant $(P=.40)$. However, because the pre-LTx group contained more women, and men and women differ in metabolically active FFM, we also corrected the measured energy expenditure for kilograms of FFM. This showed that REE per kilogram FFM was significantly higher pre-LTX than post-LTx $(P=.01)$ (Table 2$)$.

The predicted REE values using the 4 different prediction formulas did not differ much from each other, with a mean difference of $57 \mathrm{kcal}$ (range, 10-198 kcal). In most pre-LTx patients with $C F$, the predicted REE values were lower than the measured REE. Predicted values were within $10 \%$ of measured values in only 3 out of 12 patients. Figure 1 shows the Bland-Altman graphs of REE measured ( $\mathrm{kcal} / \mathrm{d}$ ) compared with REE using equations of HB 1919, HB 1984, Schofield, and WHO (1985) in pre-LTx (open squares) and post-LTx (filled squares) patients with CF. 
Table 1 Characteristics of Pre-LTx and Post-LTx Patients With Cystic Fibrosis. ${ }^{a}$

\begin{tabular}{|c|c|c|c|}
\hline Characteristic & Pre-LTx $(n=12)$ & Post-LTx $(n=12)$ & P Value \\
\hline Female sex & $9(75)$ & $6(50)$ & .40 \\
\hline Age in years, median (IQR) & $31.6(23.3-40.0)$ & $33.5(22.3-40.3)$ & 1.00 \\
\hline Time post-LTx, median (IQR) & NA & $11.5(8.1-21.3)$ & \\
\hline $\mathrm{PI}$ & $12(100)$ & $12(100)$ & 1.00 \\
\hline CFRD & $7(58)$ & $7(58)$ & 1.00 \\
\hline NODAT & NA & $5(42)$ & \\
\hline Genotype & & & .45 \\
\hline$\Delta \mathrm{F} 508 / \Delta \mathrm{F} 508$ & $6(50)$ & $4(33)$ & \\
\hline$\Delta$ F508/other & $3(25)$ & $2(17)$ & \\
\hline$\Delta$ F508/unknown & $3(25)$ & $6(50)$ & \\
\hline Pseudomonas aeruginosa & $8(67)$ & $6(50)$ & .41 \\
\hline $\mathrm{FEV}_{1} \%$ predicted, median (IQR) & $25.0(20.3-28.5)$ & $76.5(56.0-91.8)$ & .00 \\
\hline Body weight, median (IQR), kg & $56.0(52.5-65.1)$ & $60.7(55.6-74.7)$ & .22 \\
\hline Height, median (IQR), cm & $167.3(162.8-174.1)$ & $172.5(162.1-175.8)$ & .68 \\
\hline BMI, median (IQR), kg/m² & $20.4(19.1-21.9)$ & $22.0(18.9-24.6)$ & .68 \\
\hline Low $\mathrm{BMI}<18.5 \mathrm{~kg} / \mathrm{m}^{2}$ & $1(8.3)$ & $1(8.3)$ & 1.00 \\
\hline FFM, median (IQR), kg & $41.2(39.2-46.9)$ & $45.5(43.0-59.2)$ & .22 \\
\hline
\end{tabular}

BMI, body mass index; CFRD, cystic fibrosis-related diabetes; FEV $\%$ predicted, forced expiratory volume in 1 second as percent of predicted; FFM, fat-free mass; IQR, interquartile range; LTx, lung transplantation; NA, not applicable; NODAT, new-onset diabetes after transplant; PI, exocrine pancreatic insufficiency. avalues are presented as number (\%) unless otherwise indicated.

Table 2 Measured Resting Energy Expenditure in Pre-LTx and Post-LTx Patients With Cystic Fibrosis.

\begin{tabular}{lccc}
\hline Measured REE & Pre-LTx (n=12), Mean (SD) & Post-LTx (n=12), Mean (SD) & $\boldsymbol{P}$ Value \\
\hline REE, kcal/d & $1735(251)$ & $1650(235)$ & .40 \\
REE, \% predicted HB 1919 & $123(18.6)$ & $109(7.4)$ & .04 \\
REE, \% predicted HB 1984 & $122(16.7)$ & $109(5.1)$ & .02 \\
REE, \% predicted Schofield & $124(19.4)$ & $110(6.1)$ & .02 \\
REE, \% predicted WHO & $123(19.3)$ & $109(5.7)$ & .03 \\
REE/kg, kcal/kg & $30.0(5.0)$ & $26.3(2.4)$ & .03 \\
REE/kg FFM, kcal/kg & $40.5(7.0)$ & $34.3(3.2)$ & .01 \\
\hline
\end{tabular}

FFM, fat-free mass; HB, Harris-Benedict; LTx, lung transplantation; REE, resting energy expenditure; WHO, World Health Organization. 
As can be seen in Figure 1, pre-LTX using prediction equations underestimates REE in 75\%$83 \%$ of the patients. Within 2-years post-LTX, the predicted REE values were much closer to the measured REE. In $58 \%-67 \%$ of patients, REE was estimated within $10 \%$ of the measured values.

In all 7 pre-LTx patients with CF who had CFRD, an underestimation of REE was seen using all 4 prediction equations. Six these 7 pre-LTx patients with CFRD were colonized with Pseudomonas aeruginosa. In 5 of 7 post-LTx patients with CFRD, prediction equations estimated REE within normal range. Also, the REE of 2 post-LTx patients with CFRD who were colonized with $P$ aeruginosa was estimated correctly using prediction equations. In 2 of 5 post-LTx patients with NODAT, an underestimation of REE was seen using all 4 prediction equations (data not shown).

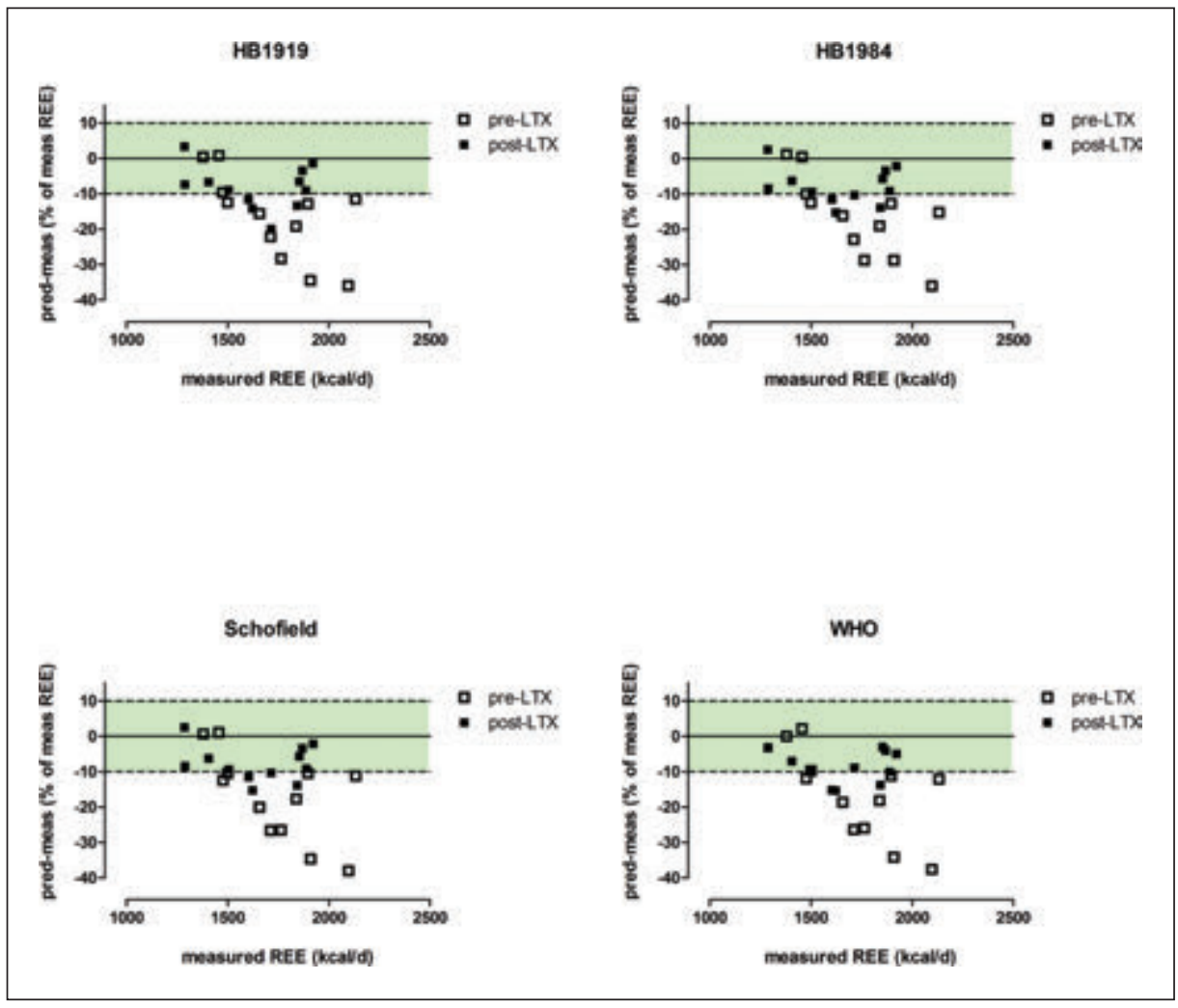

Figure 1. The difference between predicted and measured (as a percentage of measured REE) against measured REE values ( $\mathrm{kcal} / \mathrm{d}$ ) in pre-LTx (open squares) and post-LTx (filled squares) patients with cystic fibrosis. HB, Harris-Benedict; LTX, lung transplantation; REE, resting energy expenditure; WHO, World Health Organization. 


\section{DISCUSSION}

The first aim of this study was to assess the agreement between measured REE using indirect calorimetry and predicted REE according to HB 1919 and 1984, Schofield, and WHO prediction equations.

Prediction equations only estimate REE at most in $25 \%$ of pre-LTx patients with CF and $67 \%$ of post-LTx patients with CF adequately. For example, the WHO underestimates REE in 75\% of our pre-LTx patients with CF. A previous study showed underestimation by the WHO and HB 1919 equations in $>90 \%$ patients with CF with $\mathrm{PI}$ and homozygote $\triangle \mathrm{F} 508$ patients with $\mathrm{CF} .{ }^{8} \mathrm{~A}$ small Canadian study performed in 10 pre-LTx children with CF also showed underestimation of REE when predicted compared to measured ( $>115 \%$ of predicted) by using indirect calorimetry. Unfortunately, no data on prevalence of CFRD and/or $P$ aeruginosa were described. ${ }^{21}$ On the basis of these findings, we recommend measuring REE instead of using prediction formulas in patients with CF, especially pre-LTX.

The second aim of this study was to assess differences in REE in pre-LTX and post-LTx patients with CF. Increased REE is related to colonization with $P$ aeruginosa, genotype mutation, lung damage, and sex. $4,8,22,23$ In our study population, REE was underestimated using prediction equations in all pre-LTx patients with CFRD and colonized with P aeruginosa. Also, in all preLTx patients with CF with genotype mutation homozygote $\Delta F 508$, prediction equations underestimated REE. This is consistent with findings from literature, ${ }^{8}$ although 2 post-LTX patients with CF with this genotype mutation had measured REE values within the normal range of predicted values for a healthy population. Data on CFRD and NODAT were included because of its high prevalence in end-stage CF and posttransplant and the influence of CFRD on REE. ${ }^{24}$ Prediction equations underestimated REE in all but 2 pre-LTx patients with CF, who were non-CFRD patients. These findings are in concordance with literature in which elevated REE is shown in patients with CFRD compared to healthy controls. ${ }^{25}$

Within two years post-LTX, not surprisingly lung function improves in the majority of our patients with CF. In 4 out of 12 post-LTx patients with CF REE was still underestimated using prediction equations, possible due to re-colonisation with Pseudomonas aeruginosa from the patient's paranasal sinuses. ${ }^{26}$

When REE was adjusted for body weight or FFM a significant difference was seen between pre- and post-LTx patients with CF. REE/kg and REE/kg FFM was significantly higher in pre- 
than in post-LTx patients with CF. These differences may be explained by a decrease of inflammation and pulmonary infections post-LTX and a decline of increased respiratory effort. In our study blood serum C-reactive protein (CRP) values were available, but no association was found between elevated CRP values and increased REE measurement outcomes (data not shown). This is in accordance with a study in patients with chronic obstructive pulmonary disease (COPD). ${ }^{27}$ For future research in pre-LTX and post-LTx patients with CF, nitric oxide (NO) possibly may be used as a biomarker of airway inflammation. A study in 22 CF children showed that the distal airway is a major site of inflammation, and measuring alveolar NO may be a marker of distal inflammation in $\mathrm{CF}^{28}$ also used in asthma. ${ }^{29}$

Strengths of this study are the availability of REE measurements in CF patients before and after LTX. To our knowledge, this is the first study in which REE is measured in adult patients with CF before and after lung transplantation. Besides, data about infection status, CFRD and FFM was available.

This study is limited by its cross-sectional design. REE measurement could not be performed in the same patients with CF both pre- and post-LTX as in a study in 10 CF children. ${ }^{21}$ Further research is warranted using a longitudinal study design to determine changes of REE within the same patients before and after lung transplantation.

Use of BIA for measuring FFM is standard of care in our hospital. But reference values for specifically patients with CF are lacking. It is still unclear whether it is sufficiently accurate for clinical use in critically ill patients. ${ }^{30,31}$ Comparing BIA outcomes with the gold standard for indirectly measuring body composition, air displacement plethysmography, ${ }^{32}$ would contribute to the knowledge of validity of BIA in patients with CF.

Exploration of actual energy demands in pre- and post-lung transplantation patients with CF will contribute to better dietary practice. REE is an important determinant of total energy requirement in patients and with incorrect REE values from prediction equations dietitians are not able to perform an effective dietary treatment. Patient tailored REE can be used by dietitians to achieve an optimal nutritional status in this vulnerable patient population and this will additionally contribute to improvement of survival before and after lung transplantation. Our current data with future data might contribute to the development of a CF population specific prediction equation for REE also taken into account disease and activity factors. Although measuring REE gives a more reliable estimate of energy needs than prediction equations, this amount of energy may still not be enough to keep patients in energy balance. 
Losses due to absorption problems need to be taken into account, and these may be at least $10 \%$ of total energy needs. ${ }^{33}$

Research into the effects of oxygen supplementation on energy needs is lacking. Indirect calorimetry of course uses oxygen consumption and carbon dioxide production to estimate energy expenditure, and would therefore be unsuitable. Measuring total energy expenditure with the doubly-labelled water technique would overcome this problem and has the additional benefit of estimating total and not just resting energy expenditure.

Repeated weight monitoring is therefore still needed to see whether patients are in energy balance. Measurement of REE will not be necessary in patients who are perfectly able to maintain their body weight. However, in patients who lose weight - despite reporting to adhere to their diet and medication, it may be helpful in diagnosing the cause of the weight loss problem and therefore the solution. In that case, it may be more cost effective than performing a fat balance test (collecting diet information and stool samples).

In conclusion, prediction equations underestimate REE patients with end-stage CF. REE per kg body weight and REE per kg FFM is significantly higher pre-LTx as compared with postLTX. Measured REE values will contribute to better dietary treatment in patients with CF. Therefore, measurement of REE instead of using equations is recommended for patients with CF during different stages of their disease, especially pre-LTx to optimize energy requirements for improving the nutrition status. Future research should focus on explorative reasons for elevated REE.

\section{Acknowledgements}

We thank all of the participating patients with CF who made the current study possible. 


\section{REFERENCES}

1. Yen EH, Quinton H, Borowitz D. Better nutritional status in early childhood is associated with improved clinical outcomes and survival in patients with cystic fibrosis. J Pediatr. 2013; 162:530535,e1.

2. Stallings VA, Stark LJ, Robinson KA, Feranchak AP, Quinton H. Evidence-based practice recommendations for nutrition-related management of children and adults with cystic fibrosis and pancreatic insufficiency: results of a systematic review. J Am Diet Assoc. 2008;108: 832-839.

3. White H, Morton AM, Peckham DG, Conway SP. Dietary intakes in adult patients with cystic fibrosis-do they achieve guidelines? J Cystic fibrosis. 2004;3:1-7.

4. Bell SC, Bowerman AM, Nixon LE, Macdonald IA, Elborn JS, Shale DJ. Metabolic and inflammatory responses to pulmonary exacerbation in adults with cystic fibrosis. Eur J Clin Invest.2000;30:553559.

5. Schofield WN. Predicting basal metabolic rate, new standards and review of previous work. Hum Nutr Clin Nutr.1985;39(suppl 1):5-41.

6. Harris JA, Benedict FG. A biometric study of human basal metabolism. Proc Natl Acad Sci U S A.1918;4:370-373.

7. Energy and protein requirements; report of a joint FAO/WHO/UNU Expert Consultation. World Health Organization Technical Report Series.1985;724:1-206.

8. Fuster CO, Fuster GO, Galindo AD, Galo AP, Verdugo JM, Lozano FM. Analysis of energy expenditure in adults with cystic fibrosis: comparison of indirect calorimetry and prediction equations [in Spanish]. Arch Bronconeumol. 2007;43:366-372.

9. Boullata J, Williams J, Cottrell F, Hudson L, Compher C. Accurate determination of energy needs in hospitalized patients. J Am Diet Assoc. 2007;107: 393-401.

10. Blasco Redondo R. Resting energy expenditure; assessment methods and applications. Nutr Hospital.2015;31(suppl 3): 245-254.

11. Hollander FM, van Pierre DD, de Roos NM, van de Graaf EA, lestra JA. Effects of nutritional status and dietetic interventions on survival in cystic fibrosis patients before and after lung transplantation. J Cystic Fibrosis. 2014;13:212-218.

12. Allen JG, Arnaoutakis GJ, Weiss ES, Merlo CA, Conte JV, Shah AS. The impact of recipient body mass index on survival after lung transplantation. J Heart Lung Transplant. 2010;29:1026-1033.

13. Psota T, Chen KY. Measuring energy expenditure in clinical populations: rewards and challenges. Eur J Clin Nutr. 2013;67:436-442.

14. Moudiou T, Galli-Tsinopoulou A, Vamvakoudis E, Nousia-Arvanitakis S. Resting energy expenditure in cystic fibrosis as an indicator of disease severity. J Cystic Fibrosis. 2007;6: 131-136.

15. Lee TW, Brownlee KG, Conway SP, Denton M, Littlewood JM. Evaluation of a new definition for chronic Pseudomonas aeruginosa infection in cystic fibrosis patients. J Cystic Fibrosis. 2003;2:2934.

16. World Health Organization. Obesity: Preventing and Managing the Global Epidemic.Geneva, Switzerland: World Health Organization; 2000.

17. Kyle UG, Schutz Y, Dupertuis YM, Pichard C. Body composition interpretation: contributions of the fat-free mass index and the body fat mass index. Nutrition. 2003;19:597-604. 
18. Weir JB. New methods for calculating metabolic rate with special reference to protein metabolism. 1949. Nutrition. 1990;6:213-221.

19. Weijs PJ, Kruizenga HM, van Dijk AE, et al. Validation of predictive equations for resting energy expenditure in adult outpatients and inpatients. Clin Nutr. 2008;27:150-157.

20. Frankenfield D, Roth-Yousey L, Compher C. Comparison of predictive equations for resting metabolic rate in healthy nonobese and obese adults: a systematic review. J Am Diet Assoc. 2005;105:775-789.

21. Kalnins D, Pencharz PB, Grasemann H, Solomon M. Energy expenditure and nutritional status in pediatric patients before and after lung transplantation. J Pediatrics. 2013;163:1500-1502.

22. Allen JR, McCauley JC, Selby AM, et al. Differences in resting energy expenditure between male and female children with cystic fibrosis. J Pediatr. 2003;142:15-19.

23. Dorlochter L, Roksund O, Helgheim V, Rosendahl K, Fluge G. Resting energy expenditure and lung disease in cystic fibrosis. J Cystic Fibrosis. 2002;1:131-136.

24. Belle-van Meerkerk G, van de Graaf EA, Kwakkel-van Erp JM, et al. Diabetes before and after lung transplantation in patients with cystic fibrosis and other lung diseases. Diabetic Med. 2012;29: e159-e162.

25. Ward SA, Tomezsko JL, Holsclaw DS, Paolone AM. Energy expenditure and substrate utilization in adults with cystic fibrosis and diabetes mellitus. Am J Clin Nutr. 1999;69:913-919.

26. Aanaes K. Bacterial sinusitis can be a focus for initial lung colonisation and chronic lung infection in patients with cystic fibrosis. J Cystic Fibrosis. 2013;12(suppl 2)S1-S20.

27. Kao CC, Hsu JW, Bandi V, Hanania NA, Kheradmand F, Jahoor F. Resting energy expenditure and protein turnover are increased in patients with severe chronic obstructive pulmonary disease. Metabolism. 2011;60:1449-1455.

28. Suri R, Paraskakis E, Bush A. Alveolar, but not bronchial nitric oxide production is elevated in cystic fibrosis. Pediatr Pulmonol. 2007;42:1215-1221.

29. Paredi P, Kharitonov SA, Meah S, Barnes PJ, Usmani OS. A novel approach to partition central and peripheral airway nitric oxide. Chest. 2014;145:113-119.

30. Malbrain ML, Huygh J, Dabrowski W, De Waele JJ, Staelens A, Wauters J. The use of bio-electrical impedance analysis (BIA) to guide fluid management, resuscitation and deresuscitation in critically ill patients: a bench-to-bedside review. Anaesthesiol Intensive Ther. 2014;46:381-391.

31. Alicandro G, Battezzati A, Bianchi ML, et al. Estimating body composition from skinfold thicknesses and bioelectrical impedance analysis in cystic fibrosis patients. J Cystic Fibrosis. 2015;14:784-791.

32. Muller MJ, Braun W, Pourhassan M, Geisler C, Bosy-Westphal A. Application of standards and models in body composition analysis [published online November 6, 2015]. Proc Nutr Soc.

33. Pencharz PB, Durie PR. Pathogenesis of malnutrition in cystic fibrosis, and its treatment. Clin Nutr. 2000;19:387-394. 
蛋

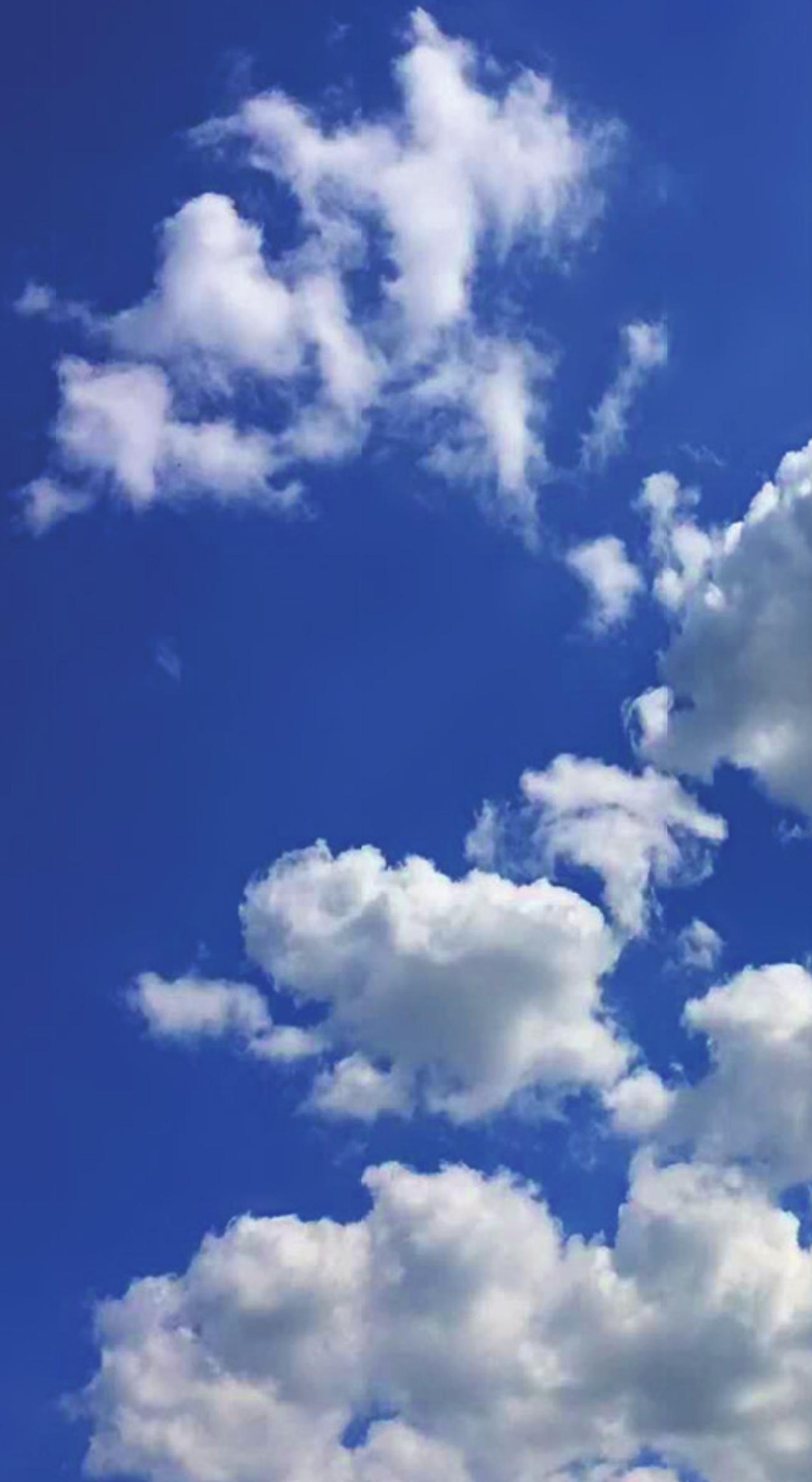




\section{Chapter 4}

Non-fasting bioelectrical impedance analysis in cystic fibrosis: Implications for clinical practice and research

F.M. Hollander-Kraaijeveld

$Y$. Lindeman

N.M. de Roos

M. Burghard

E.A. van de Graaf

H.G.M. Heijerman

Journal of Cystic Fibrosis 2020 Jan;19(1):153-158 


\section{ABSTRACT}

\section{Background}

Nutritional status affects pulmonary function in cystic fibrosis (CF) patients and can be monitored by using bioelectrical impedance analysis (BIA). BIA measurements are commonly performed in the fasting state, which is burdensome for patients. We investigated whether fasting is necessary for clinical practice and research.

\section{Methods}

Fat free mass (FFM) and fat mass (FM) were determined in adult CF patients ( $n=84$ ) by whole body single frequency BIA (Bodystat 500 ) in a fasting and non-fasting state. Fasting and non-fasting BIA outcomes were compared with Bland-Altman plots. Pulmonary function was expressed as Forced Expiratory Volume at 1 second percentage predicted (FEV1\%pred). Comparability of the associations between fasting and non-fasting body composition measurements with FEV1\%pred was assessed by multiple linear regression.

\section{Results}

Fasting FFM, its index (FFMI), and phase angle were significantly lower than non-fasting estimates $\left(-0.23 \mathrm{~kg}, \mathrm{p}=0.006,-0.07 \mathrm{~kg} / \mathrm{m}^{2}, \mathrm{p}=0.002,-0.10^{\circ}, \mathrm{p}=0.000\right.$, respectively). Fasting FM and its index (FMI) were significantly higher than non-fasting estimates $(0.22 \mathrm{~kg}, \mathrm{p}=0.008)$ $0.32 \%, p=0.005$, and $0.07 \mathrm{~kg} / \mathrm{m}^{2},(p=0.005)$. Differences between fasting and non-fasting FFM and FM were $<1 \mathrm{~kg}$ in $86 \%$ of the patients. FFMI percentile estimates remained similar in $83 \%$ of the patients when measured after nutritional intake. Fasting and non-fasting FFMI showed similar associations with FEV1\%pred ( $\beta: 4.3 \%, 95 \% \mathrm{Cl}: 0.98,7.70$ and $\beta: 4.6 \%, 95 \% \mathrm{Cl}: 1.22$, 8.00 , respectively).

\section{Conclusion}

Differences between fasting and non-fasting FFM and FM were not clinically relevant, and associations with pulmonary function remained similar. Therefore, BIA measurements can be performed in a non-fasting state. 


\section{INTRODUCTION}

Slowing the progression of lung disease severity is the primary aim in cystic fibrosis (CF) patient care, as a deterioration in pulmonary function impairs health-related quality of life and survival. ${ }^{1,2}$ Nutritional status is related to lung disease severity in CF patients. ${ }^{1-4}$ Body mass index (BMI) is often used to determine nutritional status, ${ }^{2,3}$ but does not distinguish between the amount of fat free mass (FFM) and fat mass (FM) ${ }^{5,6}$ The amount of FFM and FM is important, because CF patients with a higher FFM (in kg) have a better pulmonary function than patients with a higher FM (in kg and \%). ${ }^{6,7}$ This indicates the need for a more detailed assessment of body composition in this patient group, because CF patients may have a normal BMI, but can still have an unfavorable proportion of FFM and FM characterized by a higher amount of FM than FFM. ${ }^{6,7}$

Bioelectrical impedance analysis (BIA) is a quick and portable method, ${ }^{8,9}$ which estimates the amount of FFM and FM from measured impedance and resistance. ${ }^{8,10,11}$ Being in a nonfasting state has been shown to increase FFM and decrease FM estimates, 9,12,13 and is therefore avoided in scientific studies. However, it is unclear whether this strict condition is needed in clinical practice. ${ }^{12,14}$ Currently, patients are asked to refrain from eating and drinking until BIA measurements have been performed, which can be experienced as burdensome. Therefore, this study assessed whether the differences between fasting and non-fasting BIA results are clinically relevant in adult CF patients. Researchers may use data registered in electronic patient records for studies. Thus, for applicability in clinical research, this study additionally investigated whether associations between BIA results with pulmonary function are different when using fasting or non-fasting data.

\section{SUBJECTS AND METHODS}

\section{Study participants and design}

An observational cross-sectional study was conducted among adult CF patients. Patient recruitment was done at the outpatient clinic of the CF center at the University Medical Center Utrecht from September 2017 until July 2018. Measurements were performed as part of routine CF care at their annual check-up, as this was the convenience of the activities being undertaken at the outpatient clinic visit. The annual check-up includes an oral glucose tolerance test (OGTT) for screening for CF-related diabetes in all patients not previously diagnosed with CFRD. Patients were assigned to a private room at the outpatient clinic at 
7.45 AM., and remained there during all undertaken measurements and visits of the different specialists until 12.00 PM. Inclusion criteria were: $\geq 18$ years of age, diagnosed with CF by genotyping, ${ }^{15}$ registered at CF center Utrecht. Exclusion criteria included: pregnancy and wearing a biosensor for treatment of cystic fibrosis related diabetes (CFRD). Patients provided written informed consent. The Medical Ethical Committee of the University Medical Center Utrecht approved the study protocol (research protocol 17-915/C).

\section{Patient characteristics}

Height was measured with a wall-mounted measuring tape (SECA 206, Hamburg, Germany) to the nearest $0.1 \mathrm{~cm}$ without shoes. Weight was measured to the nearest $0.1 \mathrm{~kg}$ on a digital scale (SECA 770, Hamburg, Germany) with patients dressed in light clothes and without shoes.

Other patient characteristics were obtained from electronic medical records and included: age, $\mathrm{BMI}\left(\mathrm{kg} / \mathrm{m}^{2}\right.$ ), genotype of the disease (homozygote $\Delta \mathrm{F} 508$, heterozygote $\Delta \mathrm{F} 508$, or other), CFRD, pancreatic insufficiency (PI), presence of chronic Pseudomonas aeruginosa (P aeruginosa) infection, and use of CFTR modulator therapy (ivacaftor and combination lumacaftor/ ivacaftor). Patients were categorized based on the ESPEN BMI target value (for men $\geq 23 \mathrm{~kg}$ / $\mathrm{m}^{2}$, for women $\left.\geq 22 \mathrm{~kg} / \mathrm{m}^{2}\right) .^{5}$

\section{Non-fasting energy and fluid intake}

Nutritional intake was registered before the non-fasting BIA measurements were performed and missing values were present in only six patients. Patients who were not diagnosed with CFRD had to perform an OGTT during their check-up for diagnosis of CFRD. ${ }^{2}$ Therefore, intake included $75 \mathrm{~g}$ of glucose (300 kcal) plus $200 \mathrm{~mL}$ of water in 71 patients who had to perform an OGTT for diagnosis of CFRD. ${ }^{2}$ The OGTT was performed after patients underwent fasting BIA measurements. Nutrition and fluid intake by patients was only allowed after OGTT result and before non-fasting BIA performance. Calculation of energy (kcal) and fluid $(\mathrm{mL})$ intake was performed in eMagister (version VWB.2.2.14R1.5, Pink Roccade Healthcare, Den Bosch, NL), by using food composition data of NEVO2010 (version June 2010, National Institute for Public Health and Environment, Bilthoven, NL).

\section{Pulmonary function}

Pulmonary function was expressed as Forced expiratory volume at 1 second percentage predicted (FEV1\%pred). FEV1\%pred was assessed by spirometry tests (Geratherm, Geschwenda, Germany), ${ }^{3}$ using Global Lung Function Initiative reference equations. ${ }^{16}$ 


\section{Bioelectrical impedance analysis}

Whole-body single frequency (SF) (50 kHz) BIA measurements were performed according to Standard Operating Procedures by trained personnel, using Bodystat 500 (Bodystat Ltd, Isle of Man, British Islands). Patients laid in supine position on the examination table, and had their right hand and foot cleaned with alcohol. For each patient two new electrodes were placed on the right hand, and two new electrodes on the right foot. ${ }^{17}$ Fasting measurements were performed between 8 and 9 AM. Patients were asked to void before fasting measurements. Non-fasting measurements were between 10 and 11 AM with no restrictions regarding caffeine intake and emptying the bladder. All patients performed both fasting and non-fasting measurements.

Raw BIA data (impedance, resistance, reactance, and phase angle) were registered in electronic medical records. Estimates of FFM (in $\mathrm{kg}, \%$, and $\mathrm{kg} / \mathrm{m}^{2}$ ), and FM (in $\mathrm{kg}, \%$, and $\mathrm{kg} / \mathrm{m}^{2}$ ) were obtained, using the Kyle equation. ${ }^{18}$

Percentile estimates for the fat free mass index (FFMI) $\left(\mathrm{kg} / \mathrm{m}^{2}\right)$, and fat mass index (FMI) (kg/ $\mathrm{m}^{2}$ ), based on Schutz et al, ${ }^{19}$ were used to compare anthropometric outcomes of patients to healthy people of similar age, and gender in clinical practice. Values between the $5^{\text {th }}$ and $95^{\text {th }}$ percentile were considered as normal. ${ }^{19,20}$ Values at the $5^{\text {th }}$ percentile were considered as critical values for risk of malnutrition.

\section{Statistical analyses}

Descriptive statistics were used for the population characteristics, which were presented as means \pm standard deviation (SD) or number (\%), unless stated otherwise.

The differences between fasting and non-fasting measurements with their limits of agreement (LOA, means $\pm 1.96 \mathrm{SD}$ ) were presented in Bland-Altman graphs. ${ }^{21}$ Clinically acceptable ranges, determined using Kyle et al., ${ }^{20}$ and Schutz et al. ${ }^{19}$ percentiles, were used to define clinical relevance of the differences: -1.50 to $1.50 \mathrm{~kg}$ for FFM (kg), -1.40 to $1.40 \mathrm{~kg}$ for FM (kg), -2.0 to $2.0 \%$ for FM (\%), -0.42 to $0.42 \mathrm{~kg} / \mathrm{m}^{2}$ for FFMI, and -0.50 to $0.50 \mathrm{~kg} / \mathrm{m}^{2}$ for FMI. Clinical acceptable ranges were based on the differences between the $5^{\text {th }}$ and $10^{\text {th }}$ percentile values for the age categories 18-34 years, 35-54 years, and 55-74 years. Next, a grand mean was calculated for the differences of all age categories together. Also, to indicate significant differences between fasting and non-fasting measurements, $p$-values were generated with the paired $t$-test. 
Multiple linear regression assessed effects of using fasting or non-fasting BIA outcomes (BMI, FFMI, FMI) on associations with pulmonary function. Outcome variable was FEV1\%pred. FMI was in the model with FFMI. Age was dichotomized based on the median value for regression analyses ( $\leq$ and $>26.0$ years), which decreased the residual sums of squares. Covariates were progressively entered in the models, and remained present if the regression coefficients changed $>10 \%$. Covariates considered were: age, sex,,$^{4,7}$ presence of chronic $P$ aeruginosa infection, Pl, genotype of the disease, CFRD, ${ }^{4}$ and CFTR modulator therapy use. ${ }^{22,23}$ Ultimately, age, gender and presence of chronic $P$ aeruginosa infection remained present as covariates.

Because nutritional intake between measurements was different between patients, intakerelated bias was assessed by stratification, based on the calculated median energy ( $\leq$ or $>$ $638 \mathrm{kcal}$ ) and fluid intake ( $\leq$ or $>390 \mathrm{~mL}$ ).

All tests were two-sided and the significance level was set at 5\%. Statistical analyses were performed using IBM SPSS Statistics (version 25.0.0; IBM Corp., Armonk, NY).

\section{RESULTS}

The annual check-up included 128 patients. We excluded 44 patients from the study population, due to no consent given $(n=2)$, measurement errors (phase angle $\left.>10^{\circ} 10,11\right)(n=2)$ or because they did not have both fasting and non-fasting measurements $(n=40)$. Ultimately, 84 patients were included for analyses. Missing data for nutritional intake were only present in six patients, and were imputed using the median value.

Median (IQR) age was 26.0 years (22.0 - 33.8), and about half of the patients were men (61\%) (Table 1). Most patients (57\%) were homozygous for $\Delta$ F508 mutation, and $42 \%$ of the patients met the ESPEN BMI target value. ${ }^{2}$ Nutritional intake before non-fasting BIA measurements was $662 \mathrm{kcal} \pm 255$, and fluid intake was $420 \mathrm{~mL} \pm 183$. FEV1\%pred was $67.7 \% \pm 22.4$ (Table 1). 
Table 1. Characteristics of included adult cystic fibrosis patients $(n=84)$ and excluded adult cystic fibrosis patients $(n=40)$.

\begin{tabular}{lll}
\hline & $\begin{array}{l}\text { Included patients } \\
\mathbf{N = 8 4}\end{array}$ & $\begin{array}{l}\text { Excluded patients } \\
\mathbf{N}=\mathbf{4 0}\end{array}$ \\
\hline Age (years), median (IQR) & $26.0(22.0-33.8)$ & $29.0(24.0-37.0)$ \\
Male, number (\%) & $51(61 \%)$ & $18(45 \%)$ \\
Height (cm), mean \pm SD & $174.5 \pm 8.6$ & $171.5 \pm 8.8$ \\
Weight (kg), median (IQR) & $67.6(58.5-75.4)$ & $64.3(58.3-70.2)$ \\
BMI (kg/m), mean \pm SD & $22.3 \pm 3.1$ & $21.8 \pm 3.9$ \\
ESPEN BMI targeta ${ }^{2}$ number (\%) & $35(42 \%)$ & $10(25 \%)$ \\
PI, number (\%) & $75(90 \%)$ & $34(85 \%)$ \\
CFRD, number (\%) & $13(16 \%)$ & $25(63 \%)$ \\
$\Delta$ F508 homozygous, number (\%) & $48(57 \%)$ & $23(58 \%)$ \\
$\Delta$ F508 heterozygous, number (\%) & $30(36 \%)$ & $11(28 \%)$ \\
Other, number (\%) & $5(6 \%)$ & $6(15 \%)$ \\
CFTR modulator therapy user, number (\%) & $43(51 \%)$ & $16(40 \%)$ \\
FEV1\%pred (\%), mean \pm SD & $67.7 \pm 22.4$ & $63.7 \pm 22.0$ \\
\hline
\end{tabular}

$\mathrm{n}=$ number of subjects. $\mathrm{IQR}=$ interquartile range. $\mathrm{SD}=$ standard deviation. $\mathrm{BMI}=$ body mass index. $\mathrm{PI}=$ pancreas insufficiency. CFRD = cystic fibrosis related diabetes. CFTR = cystic fibrosis transmembrane conductance regulator. CFTR modulator therapy includes ivacaftor and lumacaftor/ivacaftor use. Normally distributed data presented as mean $\pm \mathrm{SD}$, not normally distributed data as median (IQR).

a ESPEN BMI target: $\geq 23 \mathrm{~kg} / \mathrm{m}^{2}$ for men and $\geq 22 \mathrm{~kg} / \mathrm{m}^{2}$ for women.

\section{Differences between fasting and non-fasting BIA outcomes}

On average, fasting FFM $(\mathrm{kg})$, FFMI $\left(\mathrm{kg} / \mathrm{m}^{2}\right)$, and phase angle $\left(^{\circ}\right)$ were lower than non-fasting estimates (mean difference: $-0.23 \mathrm{~kg}$, LOA: $-2.67,2.22,-0.07 \mathrm{~kg} / \mathrm{m}^{2}, \mathrm{LOA}:-0.51,0.37$, and $-0.10^{\circ}$, LOA: -0.56, 0.36, respectively) (Figure 1, A, B, F, Table 2). Fasting FM (in kg and \%), and FMI $\left(\mathrm{kg} / \mathrm{m}^{2}\right)$ were higher than non-fasting estimates (mean differences: $0.23 \mathrm{~kg}$, LOA: $-1.10,1.55$, $0.32 \%$, LOA: $-1.65,2.28$, and $0.07 \mathrm{~kg} / \mathrm{m}^{2}$, LOA: $-0.38,0.52$, respectively) (Figure 1, C, D, E, Table 2). All mean differences were significantly different from zero (FFM $p=0.002$, FFMI $p=0.006$, FM $p=0.003$ for $k g$ and $p=0.005$ for $\%, F M I ~ p=0.005$ ) (Table 2). However, the mean differences were within the clinically acceptable range (Figure 1), and LOA were similar to the clinically acceptable range (Figure 1). 
Table 2. Anthropometric outcomes from fasting and non-fasting bioelectrical impedance analysis measurements in adult cystic fibrosis patients $(n=84)$.

\begin{tabular}{lcccc}
\hline & Fasting & Non-fasting & Mean difference & P-value \\
\hline Fat free mass $(\mathrm{kg})$ & $52.1 \pm 9.5$ & $52.3 \pm 9.6$ & $-0.23 \pm 0.67$ & 0.002 \\
Fat free mass index $\left(\mathrm{kg} / \mathrm{m}^{2}\right)$ & $17.0 \pm 2.1$ & $17.1 \pm 2.1$ & $-0.07 \pm 0.23$ & 0.006 \\
Fat mass $(\mathrm{kg})$ & $16.0 \pm 6.8$ & $15.8 \pm 6.8$ & $0.22 \pm 0.67$ & 0.003 \\
Fat mass index $\left(\mathrm{kg} / \mathrm{m}^{2}\right)$ & $5.3 \pm 2.4$ & $5.2 \pm 2.4$ & $0.07 \pm 0.23$ & 0.005 \\
Fat mass $(\%)$ & $23.3 \pm 7.7$ & $23.0 \pm 7.7$ & $0.31 \pm 1.00$ & 0.005 \\
Phase angle (degree ${ }^{\circ}$ ) & $6.5 \pm 1.0$ & $6.6 \pm 1.0$ & $-0.10 \pm 0.23$ & 0.000 \\
\hline
\end{tabular}

Data presented as mean $\pm S D$. $n=$ number of subjects. $S D=$ standard deviation. P-values indicate significant difference between fasting and non-fasting values at $5 \%$, and were calculated using the paired $t$-test.

The LOA for FFM $(\mathrm{kg})$ go out of the clinically acceptable range $(1.50 \mathrm{~kg}$, LOA: $-2.67,2.22)$ (Figure 1, A), but the majority of the patients (95\%) showed a clinically acceptable difference (difference $<1.50 \mathrm{~kg}$ ) between fasting and non-fasting FFM (kg). Moreover, $86 \%$ of the patients showed a difference $<1 \mathrm{~kg}$ between fasting and non-fasting FFM (kg) and FM (kg), and in 60\% of the patients the difference was $<0.5 \mathrm{~kg}$. The LOA for phase angle also go out of the clinically acceptable range $\left(0.25^{\circ}, \mathrm{LOA}:-0.56^{\circ}, 0.36^{\circ}\right)$, which relates to only $70 \%$ of the patients showing a clinically acceptable difference between fasting and non-fasting measurements (Figure 1, F).

Approximately $80 \%$ of the patients showed normal FFMI percentile estimates (fasting $79 \%$ and non-fasting 83\%), and normal FMI percentile estimates (fasting $89 \%$ and non-fasting $90 \%)$. Percentile estimates remained similar in $83 \%$ of the patients in both fasting and nonfasting states. These results indicate that most patients showed a similar nutritional status classification in both the fasting and non-fasting states. In $24 \%$ of the patients, a lower FM\% was observed in the non-fasting state. Furthermore, 15 patients $(18 \%)$ showed a FFMI $<5^{\text {th }}$ percentile and three of these 15 patients (20\%) showed normal FFMI in non-fasting state (data not shown).

\section{Comparison associations between fasting and non-fasting BIA results with FEV1\%pred}

Our study showed that for each $1.0 \mathrm{~kg} / \mathrm{m}^{2}$ increase in fasting FFMI, FEV1\%pred was $4.34 \%$ higher ( $\beta: 4.34,95 \% \mathrm{Cl}: 0.98,7.70$ ). This was similar to the beta coefficient for non-fasting FFMI values ( $\beta$ : 4.61, 95\% Cl: 1.22, 8.00). For FMI similar results were found between using fasting FMI values and non-fasting FMI values ( $\beta$ : $-0.35,95 \% \mathrm{Cl}:-2.82,2.31, \beta:-0.30,95 \% \mathrm{Cl}:-2.76,2.17$, respectively), as well as between fasting and non-fasting phase angle ( $\beta: 7.10 \%, 95 \% \mathrm{Cl}: 1.45$, $12.74, \beta: 7.00 \%, 95 \% \mathrm{Cl}: 1.07,12.92$, respectively). These results indicate that non-fasting BIA 
measurements can be registered in electronic patient records and that researchers may use non-fasting BIA data in observational studies.

\section{Stratified analyses}

Stratification for energy ( $\leq 638 \mathrm{kcal} n=44$ and $>638 \mathrm{kcal} n=40)$ and fluid intake $(\leq 390 \mathrm{~mL}$ $n=45$ or $>390 \mathrm{~mL} n=39$ ) showed no intake-related bias (Figure 2). Mean differences between fasting and non-fasting data were similar between patients with a low and high energy (Figure 2, A, C, E) and fluid intake (Figure 2, B, D, E). Patients with a higher nutritional intake (> 638 kcal and $>390 \mathrm{~mL}$ ) showed larger LOA than patients with a low energy or low fluid intake, indicating that individual differences were larger in patients with a higher nutritional intake than patients with a lower nutritional intake. Still, even in $>90 \%$ of the patients with a high intake the differences between fasting and non-fasting BIA measurements were clinically acceptable ( $<0.42 \mathrm{~kg} / \mathrm{m}^{2}$ for FFMI and $<0.50 \mathrm{~kg} / \mathrm{m}^{2}$ for FMI) (Figure 2). 


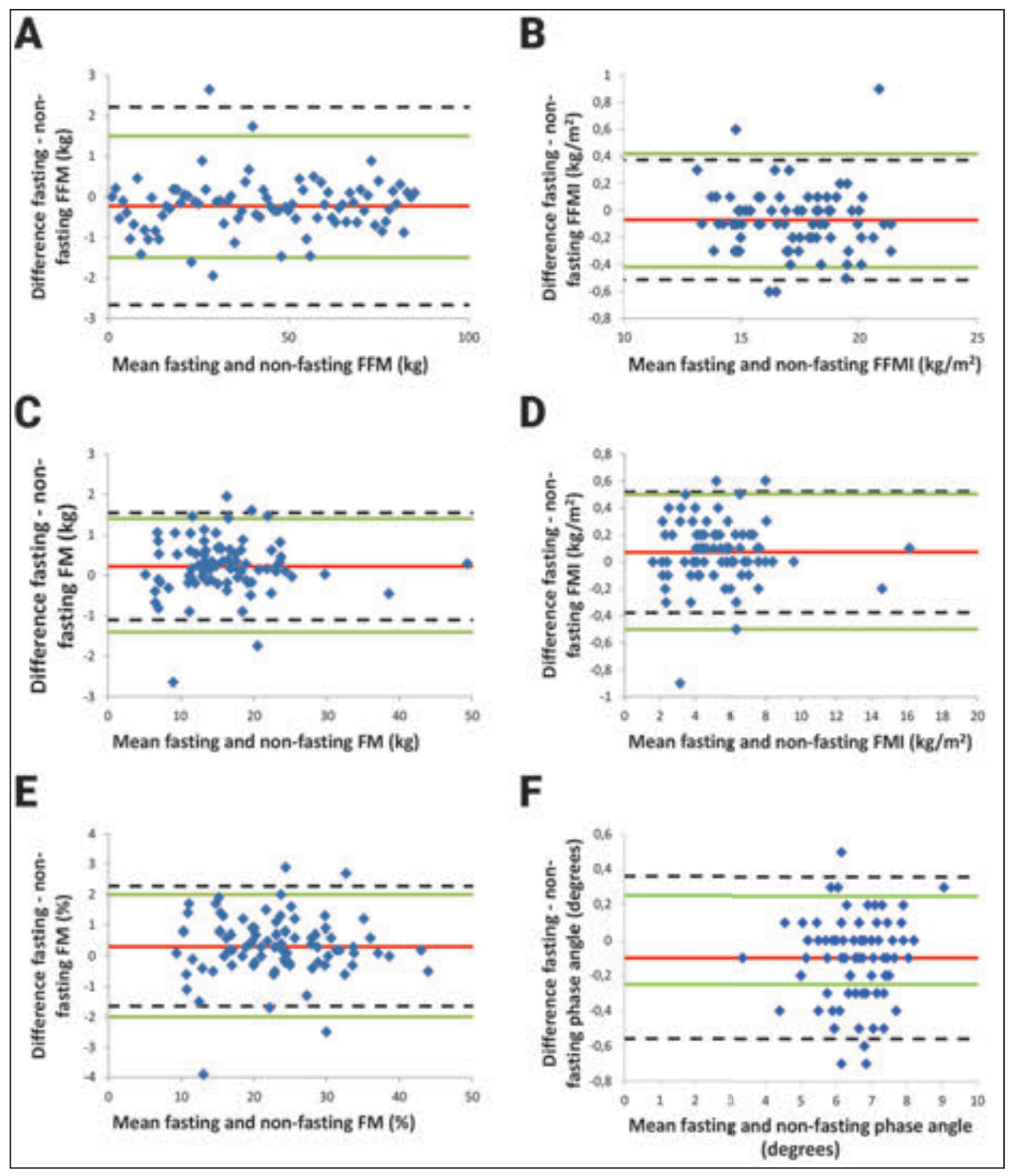

Figure 1. Bland and Altman plots showing differences between fasting and non-fasting bioelectrical impedance analysis outcomes in adult cystic fibrosis patients $(n=84)$. Red line indicates mean difference, thick dashed lines limits of agreement, thin dashed lines 95\% confidence intervals, and green lines the clinically acceptable range. FFM $=$ fat free mass. $F F M I=$ fat free mass index. FM = fat mass. FMI= fat mass index. $A$ is FFM, B is FFMI, C is FM $(\mathrm{kg}), \mathrm{D}$ is $\mathrm{FMI}, \mathrm{E}$ is $\mathrm{FM}(\%), \mathrm{F}$ is phase angle. 


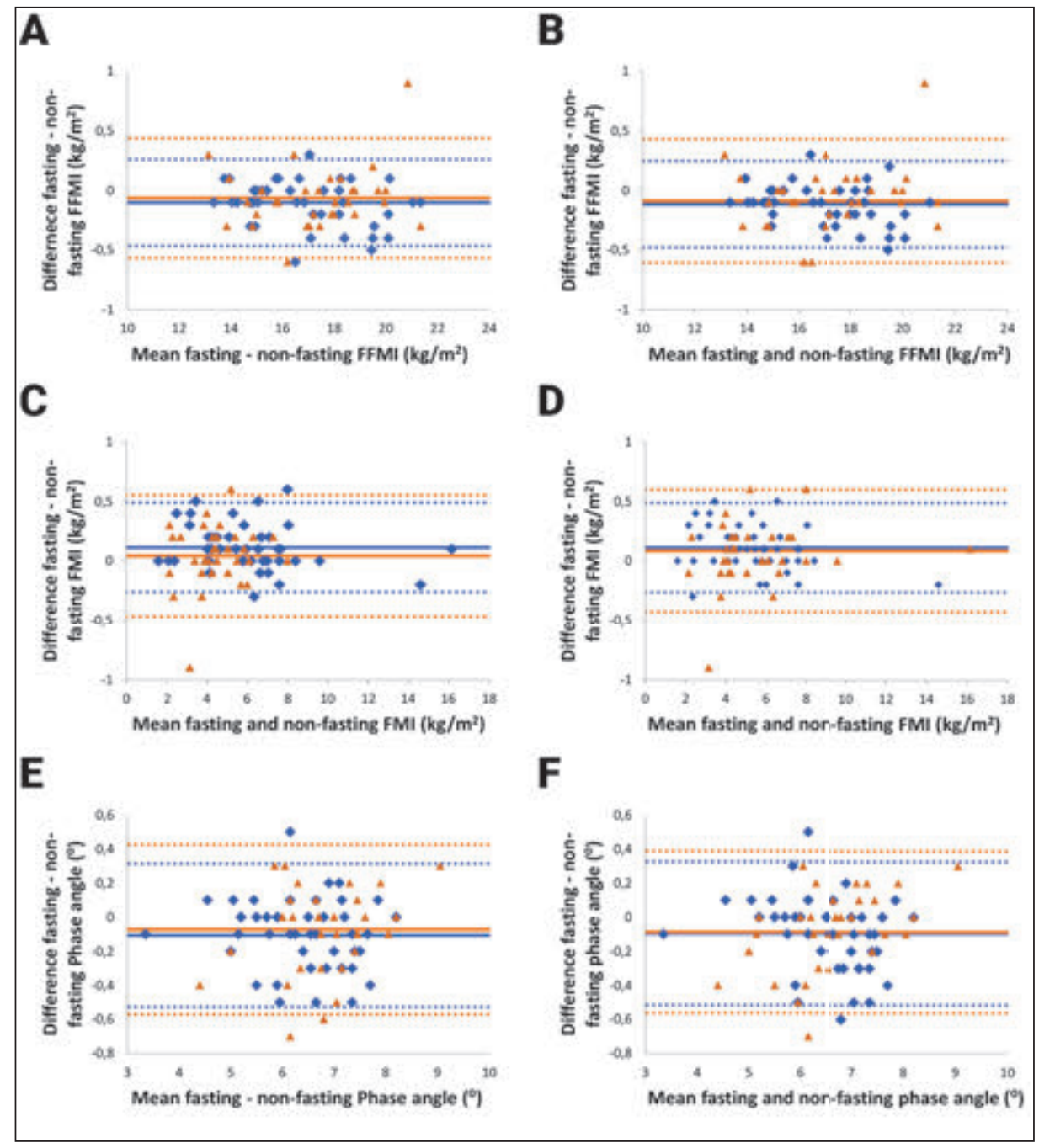

Figure 2. Bland and Altman plots showing differences between fasting and non-fasting Bioelectrical Impedance Analysis outcomes in adult cystic fibrosis patients ( $n=78)$, stratified for low ( $<664, n=45$, blue squares) and high ( $\geq 664 \mathrm{kcal}, n=33$, orange triangle) energy intake ( $A$, $C, E)$, and low ( $<423 \mathrm{~mL}, \mathrm{n}=44$, blue squares), and high ( $\geq 423 \mathrm{~mL}, \mathrm{n}=34$, orange triangle) fluid intake (B, D, F). Solid lines indicate mean differences (blue for low intake and orange for high intake), and dashed lines limits of agreement. FFMI = fat free mass index. $F M I=$ fat mass index. $\mathrm{A}=\mathrm{FFMI}$ (energy), B = FFMI (fluid), C = FMI (energy), D = FMI (fluid), E = phase angle (energy), $\mathrm{F}=$ phase angle (fluid). 


\section{DISCUSSION}

Differences between fasting and non-fasting body composition parameters in adult CF patients were not clinically relevant in $>95 \%$ of the patients, except for phase angle, and the differences were close to zero. The associations between BIA outcomes and pulmonary function were comparable when using fasting and non-fasting BIA data.

Limited studies have investigated meal effects on BIA measurements, , $14,24-26$ but none of these studies were performed in CF patients. Three of these studies reported increases in FM\% after nutritional intake, 14,24,25 which was different from our results. In contrast to our study, those previous studies used other BIA methods (segmental, multi frequency ${ }^{14}$ and leg to leg ${ }^{14,24}$ ), had subjects remain in supine position for 12 hours, ${ }^{25}$ included different time points for nonfasting measurements $\left(20,40\right.$, and 60 minutes after eating), ${ }^{14}$ or provided a higher energy intake ( $919 \mathrm{kcal}^{14}$, and $\left.869 \mathrm{kcal}^{24}\right)$. Only two studies used whole-body SF BIA outcomes; one among healthy subjects ${ }^{13}$ and one among elderly, ${ }^{26}$ and their results studies were similar to our study. The study among healthy participants observed decreases in impedance (-18 $\Omega$ ) and FM\% (-2\%) 2-4 hours after a mean intake of $652 \mathrm{kcal} .{ }^{13}$ The study among elderly observed non-significant increases in FFM (+0.2 kg) and decreases in FM $(-0.05 \mathrm{~kg})$ one hour after standardized meal consumption (299 kcal) in the morning. ${ }^{26}$ As in our study, differences were small and negligible on both the individual and group level.

Not just the mean differences between fasting and non-fasting outcomes were clinically acceptable, but also the individual differences were within the clinically acceptable range, as shown by the LOA of the Bland-Altman plots. Consequently, BIA measurements can be performed in a non-fasting state to assess anthropometric outcomes in clinical practice with $\mathrm{CF}$, preferably with similar testing conditions in order to increase reproducibility. ${ }^{9}$ Except for phase angle, for which more than $5 \%$ of the observations were observed to be clinically unacceptable. An explanation is that phase angle is directly related to shifts in body water, which slightly occurs after nutritional intake. ${ }^{13}$

In clinical practice, body composition measures are usually compared to reference values to determine malnutrition. In this study, meal consumption did not affect classification of malnutrition. Percentile estimates remained similar in $85 \%$ of the patients, indicating that the risk of malnutrition would remain the same for most patients when using non-fasting values. Fifteen patients $(18 \%)$ showed a FFMI $<5^{\text {th }}$ percentile, and 3 of these 15 patients showed normal FFMI after energy and fluid intake. However, these 3 patients showed values close to 
the critical value for risk of malnutrition in both a fasting and non-fasting state (difference from critical value $\left.\left(0.2 \mathrm{~kg} / \mathrm{m}^{2}\right)\right)$. This indicates that close monitoring of patients showing values close to the critical value of malnutrition is warranted. These results substantiate that nonfasting BIA measurements can be implemented in CF patient care.

Furthermore, this study assessed effects of using fasting and non-fasting BIA measurements on associations between body composition outcomes with pulmonary function. No differences in estimates or directions were observed between fasting and non-fasting associations with pulmonary function. This means that other observational studies that used either fasting or non-fasting values can be combined for use in meta-analyses.

Strengths of this study were the limited missing values in our dataset of the included patients. Secondly, our study population was representative of other CF study populations in terms of demographic and disease characteristics. ${ }^{4,5,7}$ Also, high prevalence of normal values for FFMI, FMI, and phase angle were observed, ${ }^{10,19}$ which can be related to inclusion of clinically stable patients. Another explanation is that $50 \%$ of the patients used CFTR modulators, which is associated with improved nutritional status. ${ }^{22}$ Moreover, CF centered care is recommended to provide an adequate treatment of CF patients and improve quality of life. ${ }^{27}$ Despite that not all patients who visit the outpatient clinic were included for analyses ( $n=84$ in the study sample versus $n=128$ in the study population), most patient and disease characteristics were similar between the study population and study sample. There was a difference between the prevalence of CFRD, our study population mainly included patients without CFRD. Patients who had been diagnosed with CFRD before were not invited for the OGTT and therefore not eligible for our study. Although this is a form of selection bias, we do not think that having CFRD or not will affect the difference in body composition before and after a meal. Patients for whom we did not have both measurements (fasting and non-fasting) were not clinically different from the ones we used in our analysis. For that reason, we think that the results can be extrapolated to the general CF population.

This study had some limitations. First, the Kyle equation to estimate body composition ${ }^{18}$ has not been validated in CF patients, but showed good precision (coefficient of variation 3.6\%, standard error of the estimate of FFM $1.72 \mathrm{~kg}$ ) when validated against Dual-energy X-ray absorptiometry among healthy individuals, as compared to other BIA equations. ${ }^{9}$ Secondly, SF BIA measurements may be less accurate than Multi Frequency BIA measurements, ${ }^{8}$ but meal effects on impedance changes have shown to be similar between SF and Multi frequency BIA in healthy participants..$^{13,14}$ Nonetheless, the results of this study should cautiously be 
interpreted when applied to MF BIA or BIS due to differences in the techniques. ${ }^{9}$ Also, it is important to acknowledge that performing BIA measurements in a non-fasting state can increase potential errors of the estimate, ${ }^{9}$ and should therefore still be cautiously interpreted. Moreover, we cannot draw conclusions regarding measuring changes over time as data were collected cross-sectionally. Though using similar testing conditions increases reproducibility. ${ }^{9}$ Another limitation of this study is that patients could ingest caffeine and were not asked to void their bladder before non-fasting measurements, which may influence the results. ${ }^{9}$ Only 6 (7\%) patients performed physical exercise between measurements, which is due to the patients schedule at the outpatient clinic. Still, none of the patients with a clinically unacceptable difference performed exercise between measurements or showed similarities in factors known to influence BIA results.

In conclusion, differences between fasting and non-fasting FFMI and FMI were not clinically relevant. Also, associations between anthropometric outcomes with pulmonary function remained similar between using fasting and non-fasting data. Therefore, assessment and monitoring of the nutritional status by using BIA measurements can be performed in a nonfasting state in adult CF patients visiting the outpatient clinic.

Competing interests No conflict of interest was reported.

Funding None.

Acknowledgements The authors thank the patients for their participation. 


\section{REFERENCES}

1. Calella P, Valerio G, Brodlie M, Donini LM, Siervo M. Cystic fibrosis, body composition and health outcomes: a systematic review. Nutrition 2018; 55(56):131-9.

2. Turck D, Braegger CP, Colombo C, Declercq D, Morton A, Pancheva R, et al. ESPEN-ESPGHANECFS guidelines on nutrition care for infants, children, and adults with cystic fibrosis. Clin Nutr 2016;35(3):557-77.

3. Castellani C, Duff AJA, Bell SC, Heijerman HGM, Munck A, Ratjen F, et al. ECFS best practice guidelines: the 2018 revision. J Cystic Fibros 2018;17(2):153-78.

4. Kerem E, Viviani L, Zolin A, MacNeill S, Hatziagorou E, Ellemunter $H$, et al. Factors associated with FEV1 decline in cystic fibrosis: analysis of the ECFS patient registry. Eur Res J 2014;43:125-33.

5. King SJ, Nyulasi IB, Strauss BJG, Kotsimbos T, Bailey M and Wilson JW. Fat-free mass depletion in cystic fibrosis: associated with lung disease severity but poorly detected by body mass index. Nutrition 2010;26(7-8):753-9.

6. Bosy-Westphal A, Müller MJ. Identification of skeletal muscle mass depletion across age and BMI groups in health and disease - there is need for a unified definition. Int J Obes (Lond) 2015;39:37986.

7. Alvarez JA, Ziegler TR, Millson EC, Stecenko AA. Body composition and lung function in cystic fibrosis and their association with adiposity and normal-weight obesity. Nutrition (Burbank, Los Angeles County, Calif) 2016;32(4):447-52.

8. Mialich MS, Sicchieri JMF, Jordao Junior AA. Analysis of body composition: a critical review of the use of bioelectrical impedance analysis. Int J Clin Nutr 2014;2(1):1-10.

9. Earthman CP. Body Composition tools for assessment of adult malnutrition at the bedside. $J$ Parenter Enter Nutr 2015;39(7):787-822.

10. Bosy-Westphal A, Danielzik S, Dorhofer RP, Later W, Wiese S, Muller MJ. Phase angle from bioelectrical impedance analysis: population reference values by age, sex, and body mass index. JPEN J Parenter Enteral Nutr 2006;30(4):309-16.

11. Lukaski HC, Kyle UG, Kondrup J. Assessment of adult malnutrition and prognosis with bioelectrical impedance analysis: phase angle and impedance ratio. Curr Opin Clin Nutr Metab Care 2017;20(5):330-9.

12. Caceres DI, Sartor-Messagi M, Rodriguez DA, Escalada F, Gea J, Orozco-Levi M, et al. Variability in bioelectrical impedance assessment of body composition depending on measurement conditions: influence of fast and rest. Nutr Hosp 2014;30(6):1359-65.

13. Slinde F, Rossander-Hulthen L. Bioelectrical impedance: effect of 3 identical meals on diurnal impedance variation and calculation of body composition. Am J Clin Nutr 2001;74(4):474-8.

14. Dixon CB, Masteller B, Andreacci JL. The effect of a meal on measures of impedance and percent body fat estimated using contact-electrode bioelectrical impedance technology. Eur J Clin Nutr 2013;67(9):950-5.

15. Farrell PM, White TB, Ren CL, Hempstead SE, Accurso F, Derichs N, et al. Diagnosis of cystic fibrosis: consensus guidelines from the cystic fibrosis foundation.J Peadiatr 2017;181(Supplement) [S4-S15. e1]. 
16. Quanjer PH, Stanojevic S, Cole TJ, Baur X, Hall GL, Culver BH, et al. Multi-ethnic reference values for spirometry for the 3-95-yr age range: the global lung function 2012 equations. Eur Respir J 2012;40(6):1324-43.

17. Khalil SF, Mohktar MS, Ibrahim F. Theory and fundamentals of bioimpedance analysis in clinical status monitoring and diagnosis of diseases. Sensors (Basel, Switzerland) 2014;14(6): 10895-928.

18. Kyle UG, Genton L, Karseard L, Slosman DO, Pichard C. Single prediction equation for bioelectrical impedance analysis in adults aged 20-94 Years. App/ Nutr Investig 2001;17(3):248-53.

19. Schutz Y, Kyle UU, Pichard C. Fat-free mass index and fat mass index percentiles in Caucasians aged 18-98 y. Int J Obes Relat Metab Disord 2002;26(7):953-60.

20. Kyle UG, Genton L, Slosman DO, Pichard C. Fat-free and fat mass percentiles in 5225 healthy subjects aged 15 to 98 years. Nutrition 2001;17(7-8):534-41.

21. Giavarina D. Understanding Bland Altman analysis. Biochem Med 2015;25(2):141-51.

22. Brodlie M, Haq IJ, Roberts K, Elborn JS. Targeted therapies to improve CFTR function in cystic fibrosis. Genome Med 2015;24(7):101.

23. Bulloch MN, Hanna C, Giovane R. Lumacaftor/ivacaftor, a novel agent for the treatment of cystic fibrosis patients who are homozygous for the F580del CFTR mutation. Expert Review of Clinical Pharamco 2017;10(10):1055-72.

24. Androutsos O, Gerasimidis K, Karanikolou A, Reilly JJ, Edwards CA. Impact of eating and drinking on body composition measurements by bioelectrical impedance. J Hum Nutr Diet. 2015;28(2):16571.

25. Slinde F, Bark A, Jansson J, Rossander-Hulthen L. Bioelectrical impedance variation in healthy subjects during $12 \mathrm{~h}$ in the supine position. Clin Nutr 2003;22(2):153-7.

26. Vilaca KH, Ferriolli E, Lima NK, Paula FJ, Moriguti JC. Effect of fluid and food intake on the body composition evaluation of elderly persons. J Nutr Health Aging 2009;13(3):183-6.

27. Castellani C, Duff AJA, Bell SC, Heijerman HGM, Munck A, Ratjen F, et al. ECFS best practice guidelines: the 2018 revision. J Cystic Fibros 2018;17(2):153-78. 
Non-fasting BIA in CF: clinical practice and research 
蛋

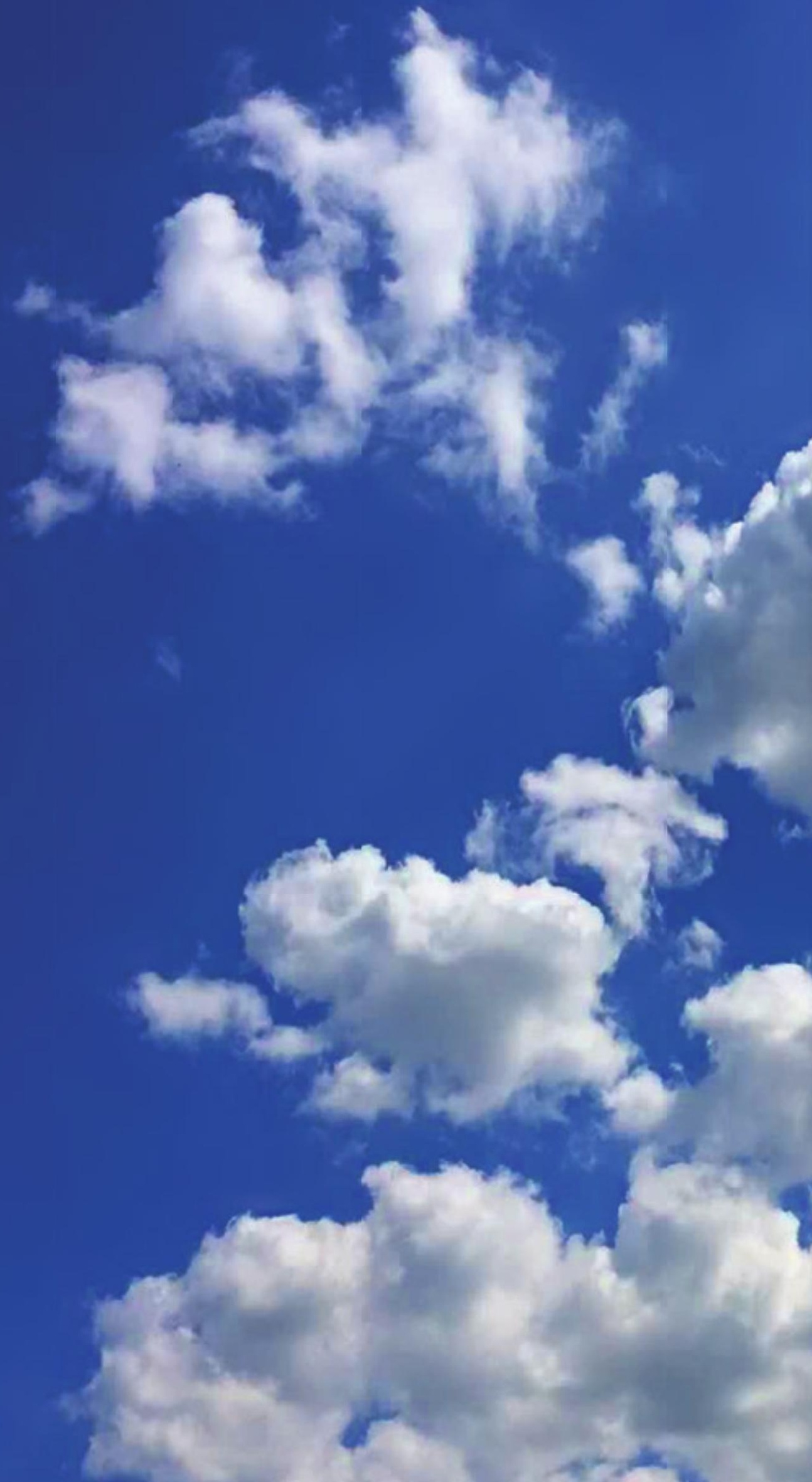




\section{Chapter 5}

Resting energy expenditure in cystic fibrosis patients decreases after lung transplantation, which improves applicability of prediction equations for energy requirement

F.M. Hollander-Kraaijeveld

A.S. van Lanen

N.M. de Roos

E.A. van de Graaf

H.G.M. Heijerman

Journal of Cystic Fibrosis accepted March 2020; in press 


\section{ABSTRACT}

\section{Background}

Resting energy expenditure (REE) is used to tailor dietary counselling in cystic fibrosis (CF) patients before and after lung transplantation (LTX). Cross-sectional studies suggest REE decreases after LTX. Follow-up data in patients are lacking. Therefore, we assessed REE in patients with CF before and after LTX, and compared measured REE to predicted REE using Harris-Benedict $(1919,1984)$, Schofield and FAO/WHO/UNU prediction equations.

\section{Methods}

REE was measured in 14 patients with CF (mean age 32, range 15 - 49 years) in fasted state using indirect calorimetry; once before and 4 times after LTX, with a 2-year follow-up. REE was expressed as $\mathrm{kcal} /$ day and $\mathrm{kcal} / \mathrm{kg}$ fat-free mass (FFM)/day. Linear mixed models were used to analyze time differences.

\section{Results}

Before LTX, mean measured REE was $1787 \mathrm{kcal} /$ day or $40 \mathrm{kcal} / \mathrm{kg}$ FFM, which was 122 - 124\% of predicted REE, depending on the prediction equation. Twelve months after $L T x$, this had significantly decreased to $1620 \mathrm{kcal} /$ day $(\mathrm{P}<.001)$ or $33 \mathrm{kcal} / \mathrm{kg} \mathrm{FFM}(\mathrm{P}<.001)$, which was 107 $108 \%$ predicted (all $P \leq .001$ ). A year after $L T x$, values stabilized and prediction was accurate (i.e. within $10 \%$ of measured REE) in about two thirds of patients. Body mass index (BMI), fat-free mass index (FFMI) and pulmonary function improved significantly after LTx (all below P=.012).

\section{Conclusions}

This longitudinal study indicates that REE in patients with CF decreases by almost $10 \%$ after LTX. Prediction equations underestimate REE in CF patients before LTX by about $20 \%$. If measuring REE is no option, we recommend using these additional percentages when calculating energy requirements. 


\section{INTRODUCTION}

Bilateral lung transplantation (LTX) is a complex but important therapeutic option for end-stage cystic fibrosis (CF) patients, with a significant risk of perioperative morbidity and mortality. ${ }^{1,2}$ Patients should be referred for LTx when pulmonary function has declined to $30 \%$ of predicted Forced Expiratory Volume in 1 second ( $F E V_{1} \%$ predicted) or when worsening rapidly. ${ }^{1}$ After LTx, pulmonary function, quality of life, and life expectancy can improve drastically. ${ }^{2,3}$

Progressive or severe malnutrition is a relative contra-indication for bilateral LTX.' For patients with $C F$, achieving or maintaining a good nutritional status may pose a challenge due to increased energy needs and nutrient losses from respiratory infection and malabsorption. Thus, specialized dietary counselling is warranted for patients with CF, especially before LTx. ${ }^{4-7}$ Current nutrition guidelines for CF advise energy intakes at $110-200 \%$ of the recommendation for healthy individuals with the same sex and size. ${ }^{8}$ However, energy requirements before and after LTX are insufficiently specified for CF lung transplant patients and currently based on recommendations for general surgical and other types of transplant patients.9,10

After LTX, the frequency of respiratory infections is reduced as a result of the removal of the infected lungs. As the physical condition of the patient after transplantation may improve, increase in exercise may also alter total energy demands. In addition, use of immunosuppressive drugs like corticosteroids may change habits with respect to food intake. These factors may contribute to the commonly observed increase in weight after LTX in patients with CF, and should be taken into account when providing dietary advice after LTx.

Energy needs are often estimated from resting energy expenditure (REE), ${ }^{11}$ multiplied with disease factors and a factor for physical activity. Also, residual malabsorption and energy losses from the gut, that can occur even in pancreatic enzyme replacement therapy (PERT) treated patients, contribute to energy needs. Indirect calorimetry (IC), which measures oxygen consumption and carbon dioxide production, is considered the golden standard to measure REE. ${ }^{12}$

When IC is not available, the REE can be predicted using various prediction equations. In current practice and clinical studies, the prediction equations of Harris-Benedict (HB)-1919,13 HB-1984, ${ }^{14}$ Schofield ${ }^{15}$ and Food and Agriculture Organization/World Health Organization/ United Nations University (FAO/WHO/UNU) ${ }^{16}$ are commonly used. Most prediction equations include sex, height, and weight. In a group of out clinic and clinic patients, Weijs et al. found 
the prediction equations of FAO/WHO/UNU and HB-1984 were most accurate (i.e. smallest prediction error). ${ }^{17}$ On an individual level, no prediction equation was considered accurate, as only half of the patients had a predicted REE within 10\% of measured REE. Besides not being accurate on an individual level, most prediction equations were validated in healthy persons, not in patients who may have higher energy demands due to severe disease. Indeed, an increased REE has been observed in end-stage CF patients, ${ }^{11,18-21}$ likely because of chronic infection, inflammation and increased work of breathing. 19,20 These increased demands are measured when assessing the REE with indirect calorimetry, but may be underestimated when using prediction equations. Indeed, REE was shown to be underestimated by prediction in patients with $C F, 11,18,19,21$ ranging from $113 \%$ to $132 \%$ of predicted REE. Nutritional advice based on underestimated energy demands may hamper achieving a good nutritional status ${ }^{22,23}$ and thus may limit eligibility for lung transplantation.

We previously found in a cross-sectional study that patients with CF before LTx failed to increase their low body weight, despite following dietetic advice and using nutritional support. After LTX, patients with CF gained weight more easily ${ }^{23}$, suggesting decreased energy demands and an improvement in appetite. Another previous cross-sectional study ${ }^{18}$ showed that measured REE per kg fat-free mass (FFM) was about 15\% lower in patients with CF who had a lung transplant compared to patients on the waiting list. However, it should be noted that these studies were all cross-sectional and the patients before LTx were not always comparable with those after LTx, limiting the strength of the conclusions. Therefore in this paper we investigate the change in REE in a longitudinal study in adult patients with CF before and after LTX. We also compare the results of the prediction equations with those of IC to determine the clinical applicability of prediction equations in adult CF lung transplant patients.

\section{METHODS}

\section{Study design and population}

All patients with CF who were selected for LTx between September 2014 and May 2017 and whose REE could be measured were included in this prospective study. Patients visited the outpatient clinic of University Medical Center Utrecht (UMCU) every 6 months for calculation of their Lung Allocation Score (LAS). The LAS takes into account various measures of a patient's health in order to direct donated organs towards the patients who would best benefit from a lung transplant. Scores range from 0 to 100 with a higher score reflecting individuals with most urgent need and greatest chance of success after transplantation. Patients with LAS > 
50 are classified as high risk in 1-year post-transplant mortality. ${ }^{24}$ Follow-up of the patients before and after lung transplantation was performed according to the lung transplantation protocol of UMC Utrecht and consisted of pulmonary function testing, blood analyses, sputum cultures, polymerase chain reaction for cytomegalovirus and the Eppstein-Barr Virus, X-rays of the chest and levels of tacrolimus. All measurements were performed at the UMC Utrecht. REE was measured during this visit before and after LTX at the following time points: 6 months (range: 0-14) before LTx and 6 months (range: 4-7), 12 months (range: 10-13), 18 months (range: 18-19) and 24 months (range: 24-26) after LTx. Patient characteristics such as age, gender, class of genotype mutation, exocrine pancreatic insufficiency (PI), presence of cystic fibrosis-related diabetes (CFRD), users of enteral tube feeding (ETF) and results of sputum cultures were extracted from electronic medical patient records. Exclusion criteria included: pneumothorax, pneumonectomy, claustrophobia and clinically unstable using continues oxygen. After lung transplantation, the patients were treated with tacrolimus, mycophenolate mofetil and prednisolone. The Medical Ethics Committee of University Medical Center Utrecht approved the study protocol (research protocol 15-418/C).

\section{Measurement of REE}

REE was measured in fasted patients by indirect calorimetry using a ventilated hood system (COSMED Quark RMR; COSMED, Rome, Italy), which was calibrated daily before measuring. Patients were measured awake in a supine position. Measurements of REE were performed according to institutional standard operating procedures (SOP). For further details we refer to the method section of our previous study. ${ }^{18}$ REE data was expressed as kcal/day and kcal/ kg FFM/day.

\section{Prediction of REE}

The predicted REE was calculated by equations of Harris-Benedict (1919 and 1984), ${ }^{13,14}$ Schofield ${ }^{15}$ and FAO/WHO/UNU. ${ }^{16}$ A predicted REE between $90 \%$ and $110 \%$ of REE measured was considered as an acceptable prediction; below $90 \%$ was considered an underestimation and above $110 \%$ an overestimation. ${ }^{25}$

\section{Anthropometrics and pulmonary function}

Measurements of body weight and height were performed in the Nutritional Assessment laboratory of the Department of Dietetics, using a calibrated electronic scale (SECA model 701; SECA, Hamburg, Germany) and stadiometer (SECA model 225). Patients were measured dressed in indoor clothing and without shoes. BMI was classified according to the WHO recommendations: underweight, $<18.5 \mathrm{~kg} / \mathrm{m}^{2}$; normal weight, $18.5-24.9 \mathrm{~kg} / \mathrm{m}^{2}$; and 
overweight, $25.0-29.9 \mathrm{~kg} / \mathrm{m}^{2} .{ }^{26}$ Fat-free mass (FFM) was measured routinely at the Department of Lung Function by whole-body single frequency $(50 \mathrm{kHz}$ ) bio-electrical impedance analysis (BIA)(Bodystat 1500; Bodystat Ltd, Douglas, UK). Patients were measured in a fasted state (no enteral intake for a minimum of 8 hours) lying in a supine position. The following values were used as cut-off points for low FFMI: $13.8 \mathrm{~kg} / \mathrm{m}^{2}$ for women aged 18-34 years, $14.4 \mathrm{~kg} /$ $\mathrm{m}^{2}$ for women aged $35-54$ years, $16.8 \mathrm{~kg} / \mathrm{m}^{2}$ for men aged $18-34$ years, and $17.2 \mathrm{~kg} / \mathrm{m}^{2}$ for men aged 35-54 years. ${ }^{27}$ Pulmonary function was measured routinely at the Department of Lung Function by spirometry, and expressed as $\mathrm{FEV}_{1} \%$ predicted, using Global Lung Function Initiative reference equations. ${ }^{28}$ Before September 2015, pulmonary function was measured with the ZAN spirometer (Oberthulba, Germany), which occurred in 5 patients, only before LTx. After September 2015, the Blue Cherry spirometer (Bad Kissingen, Germany) was used.

\section{Dietary intake}

During a dietetic consultation, patients' dietary intake expressed in kcal/day was assessed using a dietary history. Dietary intake was calculated by eMagister (version VWB.2.2.14R1.5, Pink Roccade Healthcare, Den Bosch, NL) by using food composition data of NEVO2010 (version June 2010, National Institute for Public Health and Environment, Bilthoven, NL). The use of additional oral nutritional support (ONS) or enteral tube feeding (ETF) was also documented.

\section{Statistical analysis}

Statistical analysis was performed using SPSS version 25.0 (SPSS, Inc, an IBM Company, Chicago, IL). Normality was assessed using Q-Q plots. As descriptive statistics we used means and $95 \%$ confidence intervals $(\mathrm{Cl})$.

A linear mixed model for repeated measures over time was used to test whether the REE significantly differed between different time points. The linear mixed model procedure prevents listwise deletion due to missing data. Time was included as a cofactor. The firstorder autoregressive heterogeneous covariance matrix was used to correct for heterogeneous variances at different time points. Estimated means, standard deviations (SD) and 95\% confidence intervals $(\mathrm{Cl})$ were used in tables and graphs. A $P$-value below .05 was considered to indicate statistical significance. 


\section{RESULTS}

\section{Study population}

Forty patients were selected for LTx between September 2014 and May 2017. Of these, 10 could not have REE measurements for different reasons: 5 had a clinically unstable condition (mean LAS of 82.4, range: 70.9 - 91), 3 had a pneumothorax (mean LAS of 41.6, range: 39.8 - 42.8), 1 was claustrophobic and 1 had a pneumonectomy. Figure 1 shows the flowchart of patients included and excluded for REE measurements. For the remaining patients, only 2 patients had missing data for no more than 2 time points. Finally, our study included 14 patients (Table 1); 8 women and 6 men, with a mean age of 32 (range: 15 to 49 years) and a mean LAS of 37.1 before LTx (range: 33.4 to 44.5). Seven patients (50\%) had CFRD and 13 (93\%) had PI. At the start of the study, all patients had a bacterial colonization of Staphylococcus aureus, Stenotrophomonas maltophilia, or other species. Eight (57\%) patients were colonized with $P$. aeruginosa and 2 (14\%) with M. abscessus. Twelve (86\%) patients had a class II mutation, 1 (7\%) patient had a class I mutation, and 1 (7\%) patient had a class $V$ mutation.

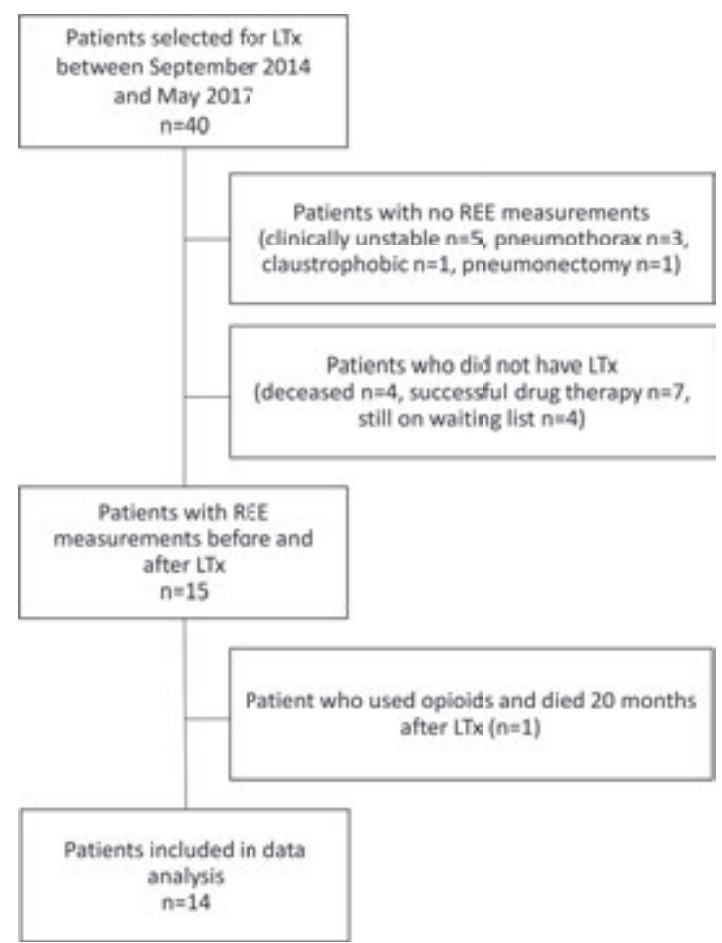

Figure 1 Flowchart of inclusion and exclusion of patients with cystic fibrosis for data analysis of REE measurements before and after lung transplantation $(n=14)$. 
Table 1. Two-year follow-up data of resting energy expenditure (kcal/d, $\mathrm{kcal} / \mathrm{d}$ per $\mathrm{kg} \mathrm{FFMl}$, body mass index, fat free mass index, pulmonary function and caloric intake in 14 adult patients with cystic fibrosis after bilateral lung transplantation.

\begin{tabular}{|c|c|c|c|c|c|}
\hline & Pre-LTx & $\begin{array}{l}6 \text { months } \\
\text { post-LTx }\end{array}$ & $\begin{array}{l}12 \text { months } \\
\text { post-LTx }\end{array}$ & $\begin{array}{l}18 \text { months } \\
\text { post-LTx }\end{array}$ & $\begin{array}{c}24 \text { months } \\
\text { post-LTx }\end{array}$ \\
\hline REE, kcal/day & $\begin{array}{c}1787 \\
(1679-1895)\end{array}$ & $\begin{array}{c}1656 \\
(1511-1801)\end{array}$ & $\begin{array}{c}1620 \\
(1538-1702)\end{array}$ & $\begin{array}{c}1608 \\
(1495-1722)\end{array}$ & $\begin{array}{c}1648 \\
(1511-1786)\end{array}$ \\
\hline REE/kg FFM, kcal/day & $\begin{array}{c}40 \\
(36-44)\end{array}$ & $\begin{array}{c}36 \\
(32-39)\end{array}$ & $\begin{array}{c}33 \\
(30-35)\end{array}$ & $\begin{array}{c}32 \\
(30-35)\end{array}$ & $\begin{array}{c}33 \\
(30-35)\end{array}$ \\
\hline $\mathrm{BMI}, \mathrm{kg} / \mathrm{m}^{2}$ & $\begin{array}{c}20.3 \\
(19.2-21.4)\end{array}$ & $\begin{array}{c}21.2 \\
(20.1-22.3)\end{array}$ & $\begin{array}{c}22.1 \\
(21.0-23.2)\end{array}$ & $\begin{array}{c}22.1 \\
(21.0-23.2)\end{array}$ & $\begin{array}{c}22.3 \\
(21.2-23.4)\end{array}$ \\
\hline FFMI, $\mathrm{kg} / \mathrm{m}^{2}$ & $\begin{array}{c}15.4 \\
(14.4-16.3)\end{array}$ & $\begin{array}{c}16.1 \\
(15.1-17.0)\end{array}$ & $\begin{array}{c}17.0 \\
(16.1-17.9)\end{array}$ & $\begin{array}{c}17.0 \\
(16.0-17.9)\end{array}$ & $\begin{array}{c}17.2 \\
(16.2-18.1)\end{array}$ \\
\hline $\mathrm{FEV}_{1} \%$ predicted & $\begin{array}{c}20.2 \\
(12.7-27.8)\end{array}$ & $\begin{array}{c}69.2 \\
(61.5-76.8)\end{array}$ & $\begin{array}{c}73.0 \\
(65.4-80.6)\end{array}$ & $\begin{array}{c}72.9 \\
(65.2-80.6)\end{array}$ & $\begin{array}{c}74.1 \\
(66.3-81.9)\end{array}$ \\
\hline Energy intake, kcal/day & $\begin{array}{c}2525 \\
(2065-2984)\end{array}$ & $\begin{array}{c}2639 \\
(2177-3102)\end{array}$ & $\begin{array}{c}2514 \\
(2054-2974)\end{array}$ & $\begin{array}{c}2464 \\
(2004-2924)\end{array}$ & $\begin{array}{c}2342 \\
(1882-2802)\end{array}$ \\
\hline
\end{tabular}

Data are expressed as estimated means from a linear mixed model analysis with $95 \%$ confidence intervals. BMI, body mass index; FAO, Food and Agriculture Organization; FEV1, Forced Expiratory Volume in 1 second; FFMI, fat-free mass index; HB, Harris-Benedict; LTX, lung transplantation; REE, resting energy expenditure; UNU, United Nations University; WHO, World Health Organization.

\section{Measured REE}

Before LTx mean REE was 1787 kcal/day (95\% Cl: 1679 - 1895) or 40 kcal/kg FFM (95\% Cl: 36 44). Twelve months after LTX, this had decreased to $1620 \mathrm{kcal} /$ day $(95 \% \mathrm{Cl}: 1538-1702, \mathrm{P}<.001)$ or $33 \mathrm{kcal} / \mathrm{kg}$ FFM (95\% Cl: 30 - 35, P<.001). REE and REE/kg FFM stabilized from 12 months post-LTX onwards (Table $\mathbf{1}$ and Figure $\mathbf{2 a}$ and $\mathbf{2} \mathbf{b}$ ). A non-significant increase in REE and REE/ kg FFM was observed from 18 to 24 months post-LTx. Mean post-LTx REE was significantly different from mean pre-LTX for all time points (all below $\mathrm{P}=.043$ )(Figure 2a). A significant decrease in REE/kg FFM was observed for all time points compared to before LTx (all below $\mathrm{P}=.009$ )(Figure $2 \mathrm{~b}$ ). 


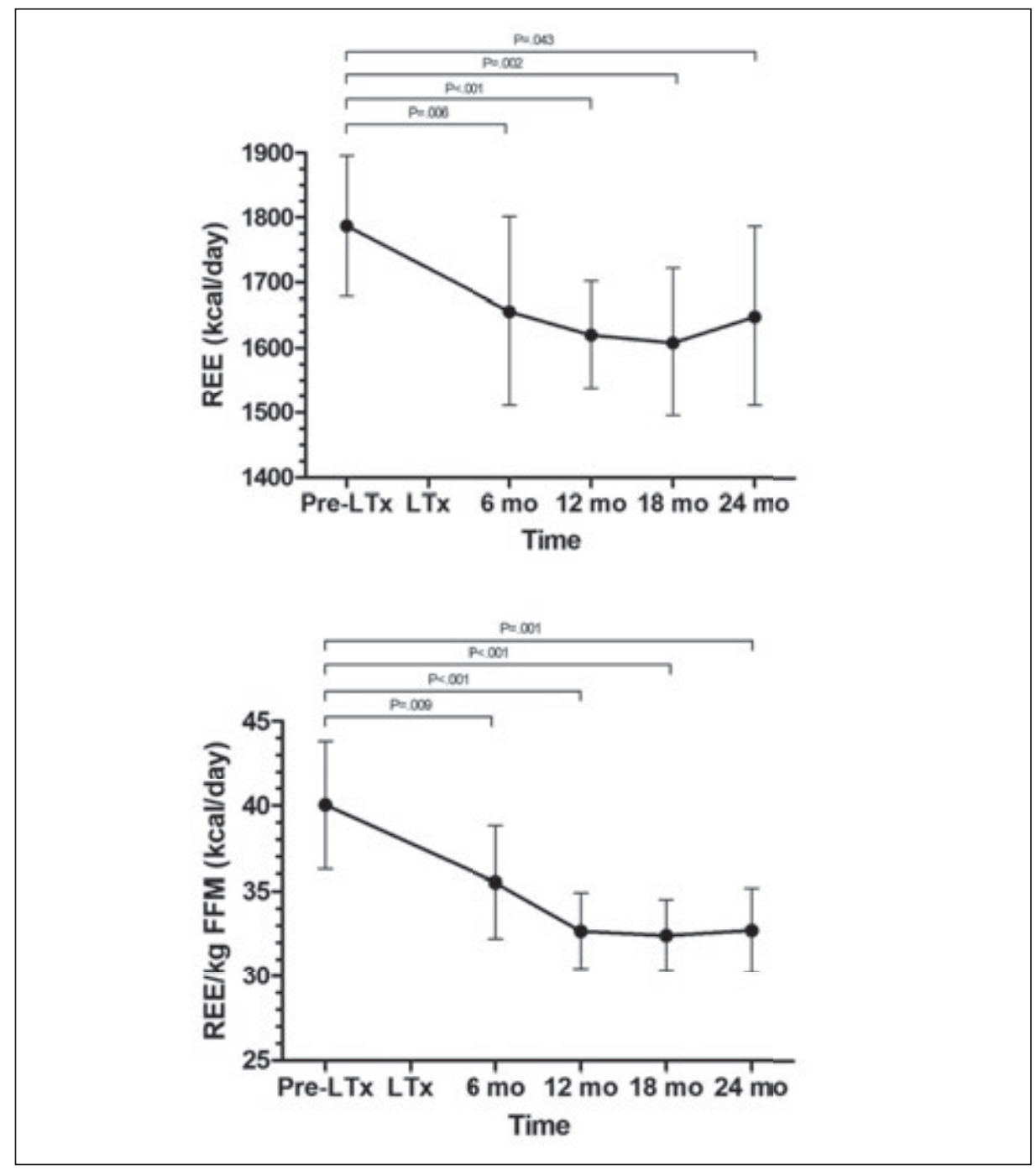

Figure $\mathbf{2 a}$ and $\mathbf{2 b}$. Mean resting energy expenditure expressed as $\mathrm{kcal} / \mathrm{day}$ (a) and $\mathrm{kcal} / \mathrm{day} /$ $\mathrm{kg}$ fat-free mass (b) in cystic fibrosis patients at five time points: one before and four after lung transplantation. Vertical bars indicate $95 \% \mathrm{Cl}$.

\section{Predicted REE}

Before LTX, the prediction equations underestimated measured REE in 11-12 of 14 patients, depending on the prediction equation used. One year after LTx this decreased to 5-6 patients, which remained stable until the last measurement (data not shown). 
When predicted REE was set as a reference value, mean measured REE was $122-124 \%$ of predicted REE before LTX, depending on which prediction equation was used (Table 1, Figure 3). Six months after LTX, this had decreased to 111-112\% (all below $P=.009$ ). Prediction was accurate from 12 months after LTx onwards, with a mean measured REE of 107-108\% predicted. A non-significant increase was observed from 18 to 24 months after LTx. Before LTX, measured REE was on average more than $120 \%$ of the predicted values, irrespective of which prediction equation was used (Figure 3). In other words, the prediction equations all underestimated measured REE and to a similar extent (mean difference between lowest and highest mean predicted value $50 \mathrm{kcal} / \mathrm{day}$ ). The largest difference that was observed in an individual patient was $114 \mathrm{kcal} /$ day. After LTx, the measured REE was close to $100 \%$ of predicted (Figure 3).

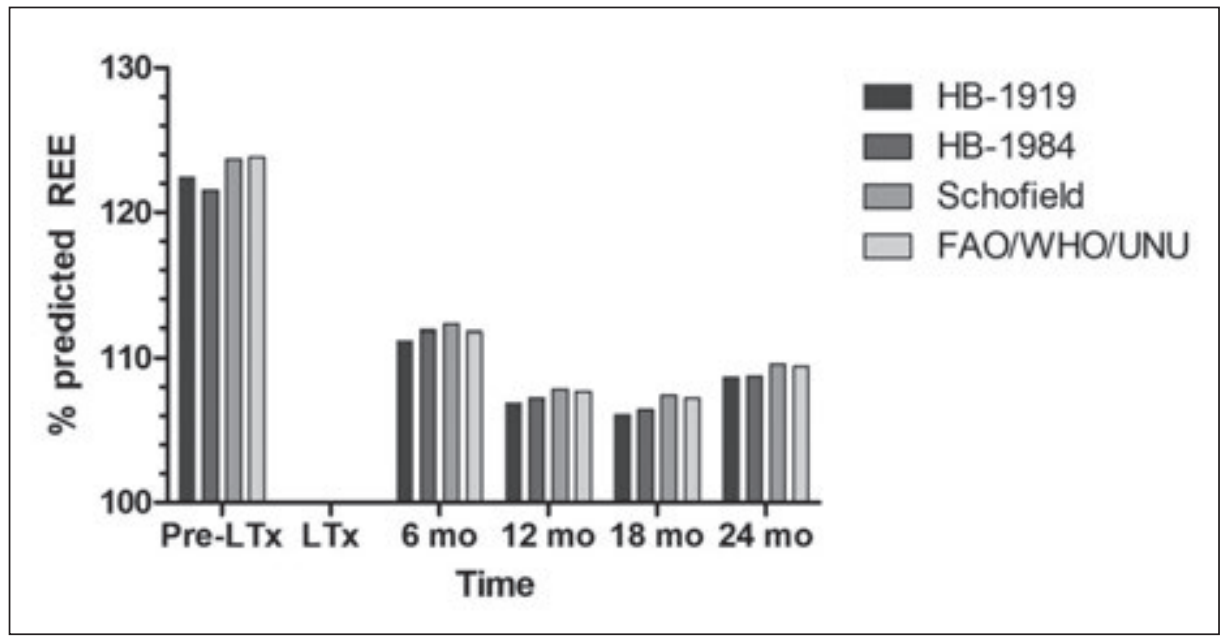

Figure 3. A comparison of mean measured resting energy expenditure as percent of predicted by 4 different prediction equations: HB-1919, HB-1984, Schofield and FAO/WHO/UNU.

\section{Anthropometrics}

Mean BMI significantly increased from $20.3 \mathrm{~kg} / \mathrm{m}^{2}$ before LTx to $22.3 \mathrm{~kg} / \mathrm{m}^{2} 24$ months after LTX $(P=.001)$ (Table 1 and Figure 4a). Underweight $\left(B M I<18.5 \mathrm{~kg} / \mathrm{m}^{2}\right)$ was observed in 1 man both before and 6 months after LTx, in 1 female 6 months post-LTx and in 1 female from 18 months post-LTX onwards. Mean FFMI significantly increased from $15.4 \mathrm{~kg} / \mathrm{m}^{2}$ before LTx to $17.2 \mathrm{~kg} / \mathrm{m}^{2}$ 24 months after LTx $(P<.001)$ (Table 1 and Figure 4b). Before LTx, 4 men and 1 female had a low FFMI according to age- and gender-specific thresholds of Schutz et al. (2002). ${ }^{27}$ Six months 
after LTx this number had decreased to 2 men and 1 female, and decreased further to 1 male and 1 female from 12 months post-LTx on.

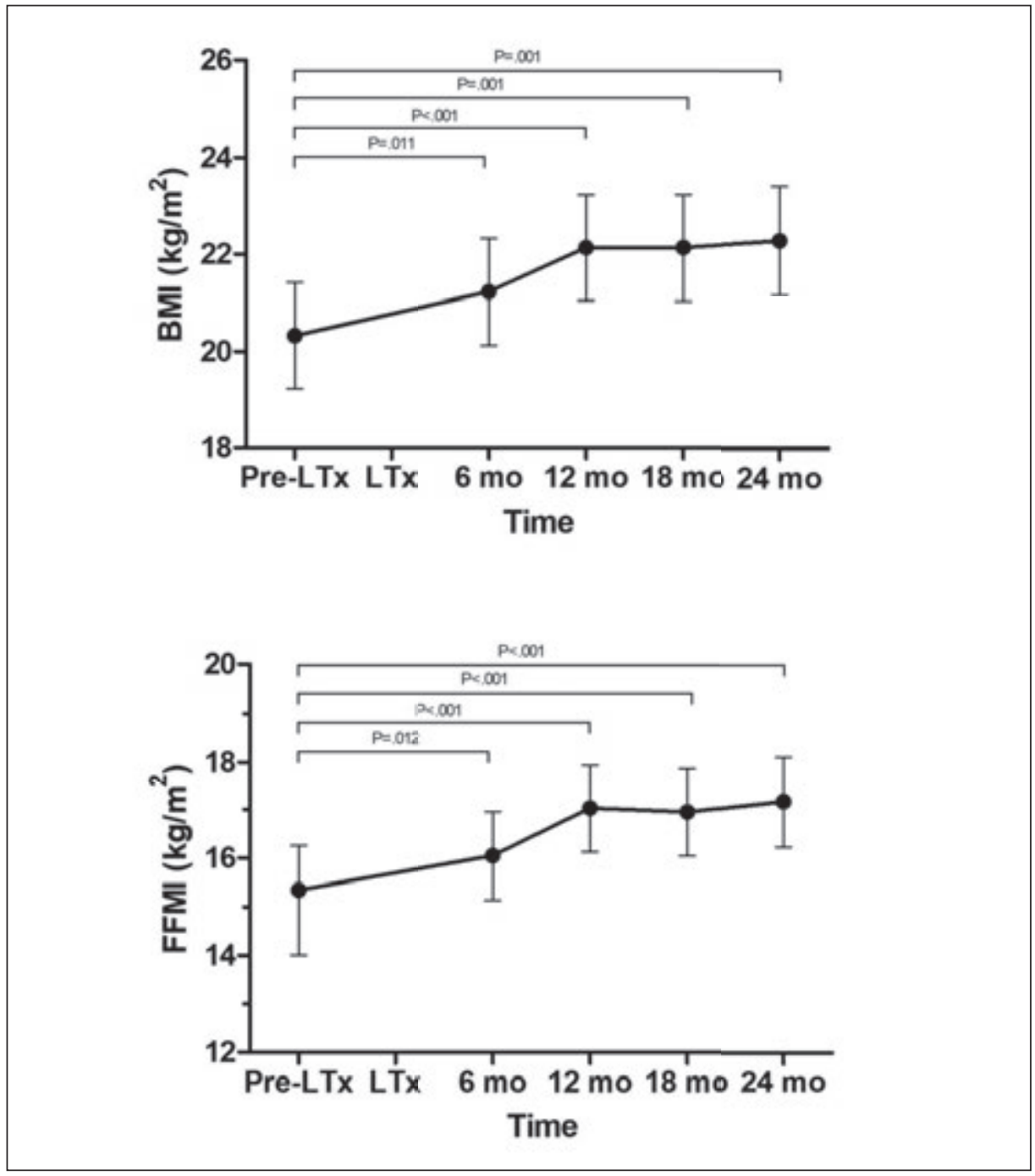

Figure $\mathbf{4 a}$ and $\mathbf{4 b}$. Body mass index and mean fat-free mass index expressed as kg per square meter in cystic fibrosis patients at five time points: one before and four after lung transplantation. Vertical bars indicate $95 \% \mathrm{Cl}$. 


\section{Pulmonary function}

Mean FEV before LTx was $20 \%$ predicted. Six months after LTx mean FEV, had increased to $69 \%$ predicted, and from 12 months after LTx on this remained stable and was approximately 73\% predicted (Table 1, Figure 5).

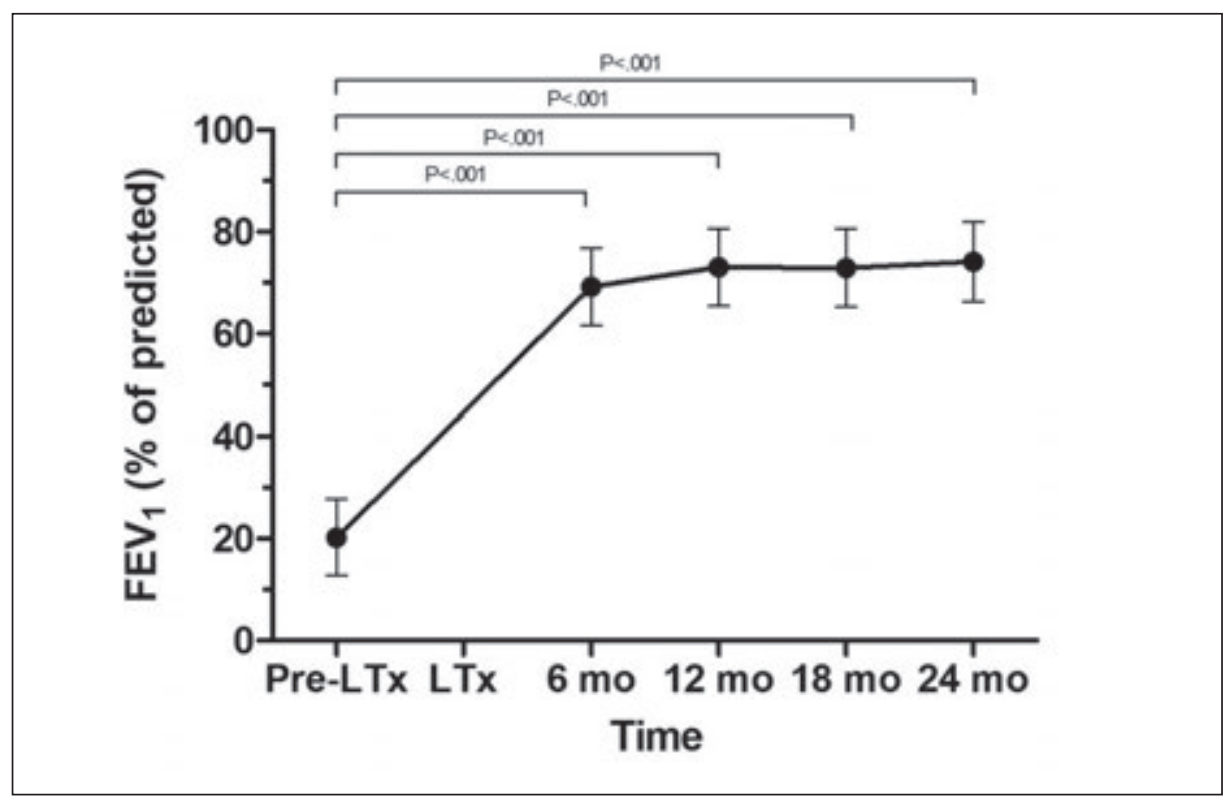

Figure 5. Forced expiratory volume in 1 second expressed as \% of predicted in cystic fibrosis patients at five time points: one before and four after lung transplantation. Vertical bars indicate $95 \% \mathrm{Cl}$.

\section{Dietary intake}

Mean energy intake estimated from the dietary history decreased from $2525 \mathrm{kcal}$ (95\% Cl: 2065 - 2984) before LTx to $2342 \mathrm{kcal}(95 \% \mathrm{Cl}$ : 1882 - 2802) 2 years after LTx (Table 1)(P=.437). Five patients (36\%) used ONS before LTX and 2 of these patients continued the use of ONS until 2 years after LTX. Seven patients (50\%) used ETF before LTX of which 3 patients by percutaneous endoscopic gastrostomy (PEG). Two patients (14\%) used a polymeric $1.5 \mathrm{kcal} /$ $\mathrm{ml}$ formula of ETF by PEG until 6 months after LTX, after which the PEG was removed. 


\section{DISCUSSION}

This longitudinal study showed that REE significantly decreased in patients with CF after LTX, compared to before LTX. Before LTX, measured REE was around $40 \mathrm{kcal} / \mathrm{kg}$ FFM, or 122-124\% predicted, depending on the equation used. From 1 year post-LTX onwards, REE and REE/kg FFM normalized, to remain stable at $33 \mathrm{kcal} / \mathrm{kg}$ FFM, which is $107-108 \%$ predicted. No clinically relevant difference was observed between REEs estimated by prediction equations of HB1919, HB-1984, Schofield and FAO/WHO/UNU. More patients had a normal BMI and FFMI after LTx than before. Mean FEV 1 increased from $20 \%$ predicted before LTx to $73 \%$ predicted from 6 months post-LTX onwards. Dietary intake did not differ significantly over time.

Only few studies investigated changes in REE. However, in none of these studies adjustments for FFM or patient characteristics were made. Therefore results are difficult to compare with our data. The change in REE per kg FFM we found in the current longitudinal study is similar to the decrease of the $6 \mathrm{kcal} / \mathrm{kg}$ FFM we found in our previous cross-sectional study comparing 12 pre-LTx patients with 12 post-LTx patients. In that study, the mean decrease in REE after LTx was $85 \mathrm{kcal} /$ day or $14 \%$ predicted REE..$^{18}$ In another longitudinal study, not in adults but in 10 pediatric CF lung transplant patients, an even larger decrease of $20 \%$ predicted after LTx was observed, compared to before..$^{19}$ However, no patient characteristics were provided, not all patients were measured in fasted state and REE was only measured at 2 time points; 8 months before and 4 months after LTX. We especially included patients with at least 2-year follow-up and 5 REE measurements, which were all performed in fasted patients.

In concordance with standard operating procedures, patients in our study were measured after an 8-hour fast. In one case, a measurement was performed after a 3-hour fast (at 24 months post-LTX) which may have slightly increased the REE due to diet-induced thermogenesis. Seven patients using CFTR modulator therapy were excluded because this medication can lower REE. ${ }^{29} \mathrm{~A}$ single patient using opioids was also excluded. Before the study we hypothesized that REE was likely to reduce due to less inflammation after LTx, but that this reduction could be compensated by an increase in muscle mass if physical activity would increase with better lung function. ${ }^{29}$ Indeed, pulmonary function was markedly improved and we did see an increase in FFM, but still REE was lower after LTX than before. This suggests that one year after LTX, the increase in REE due to a larger FFM, which is more metabolically active, is much smaller than the reduction in REE due to the declining disease factor. For future research we advise to include physical activity tracking, to better understand the impact of 
activity on the association with REE and on total energy expenditure of post lung transplant patients with CF.

The current study contained 5 patients whose REE remained higher than $110 \%$ predicted 2 years after LTX. All these patients had a homozygous $\triangle F 508$ mutation, PI, and CFRD. All but one patient had $P$. aeruginosa colonization. We speculate that the increased REE in these 5 patients 2 years after LTx may be due to a combination of the abovementioned factors. This is in accordance with literature on factors that elevate REE in patients with CF. Both pancreatic function and genotype are described as predictors of elevated REE, independent of pulmonary function. ${ }^{30}$ We found only one cross-sectional study that showed higher energy expenditure in adult patients with CF and CFRD, compared to healthy controls, but only during recovery of exercise. ${ }^{31}$ Therefore, there is insufficient evidence yet to judge whether CFRD increases REE. Intravenous treatment of $P$. aeruginosa colonization has been described previously to lower REE. ${ }^{32}$ This suggests REE is higher in patients with a $P$. aeruginosa colonization, which seems plausible. Almost two thirds of our patients had a bacterial colonization with $P$. aeruginosa. Two patients in our study received antibiotic treatment for $M$. abscessus during the follow-up period. This may have decreased REE, as antibiotic treatment for $P$. aeruginosa eradication has been reported to decrease REE. ${ }^{32,33}$ It may be difficult to distinguish between the effects of diabetes and bacterial colonization: in a previous cross-sectional study of ours, ${ }^{18}$ all 7 pre-LTX CF patients with CFRD had a higher REE than predicted, but 6 of them were also colonized with P. aeruginosa. REE was estimated within $10 \%$ of measured in all 7 post-LTx patients with CFRD, of which 2 patients were colonized with P. aeruginosa. In the current study no association was observed between C-reactive protein and REE (data not shown).

The largest limitation of our study is the small sample size, making it impossible to analyze subgroups such as patients with CFRD or patients with $P$. aeruginosa or M. abscessus. Future studies are encouraged to validate our observations and perform subgroup analyses with a larger sample size. Despite the fact that more hospitals are equipped with indirect calorimetry to measure REE, in current medical care, it is not always available in daily practice due to time and resource issues.

Measuring REE is expected to improve dietary advice and consequently nutritional status for patients with CF on the waiting list for LTX. In a previous small retrospective study, we found that a low FFMI at waiting list entry impaired survival in LTx candidates. ${ }^{23}$ Also, lean body mass depletion specifically has been associated with higher mortality before LTX. ${ }^{23,34}$ Although nutritional advice is not the only causal factor in low body weight and BMI, we expect 
that measuring REE will increase the chance of obtaining a good nutritional status, which will increase survival on the waiting list. For clinical practice, current results indicate that dietary recommendations for patients with CF need to be altered after LTx given the significant reduction in REE and improved BMI and FFMI without significant change of energy intake. In post-LTX CF nutritional management, REE estimation by prediction equations is considered accurate from one year after LTx. Hereafter, caution should be warranted advising high caloric diets to avoid redundant weight gain.

In conclusion, this 2-year follow-up study in adult patients with CF demonstrated that REE and REE/kg FFM significantly decrease after lung transplantation. From then on, REE and $\mathrm{REE} / \mathrm{kg}$ FFM remain stable and prediction using commonly used equations is accurate as in healthy individuals (i.e. within $10 \%$ of the measured value) in about two thirds of the patients. To prevent underestimation of energy needs, especially in underweight patients with CF, we stress the importance of measuring REE and advise against the use of prediction equations in patients with CF with end-stage lung disease until a year after LTX.

Declaration of competing interest The authors have no financial or other conflicts of interest to disclose. The corresponding author thanks the patients for participation in the study. Funding none 


\section{REFERENCES}

1. Weill D., Benden C., Corris P.A., Dark J.H., Davis R.D., Keshavjee S., et al. Consensus document for the selection of lung transplant candidates: 2014--an update from the Pulmonary Transplantation Council of the International Society for Heart and Lung Transplantation. J Heart Lung Transplant 2015;34(1):1-15.

2. Chambers D.C., Cherikh W.S., Goldfarb S.B., Hayes D. Jr., Kucheryavaya A.Y ., Toll A.E., et al. The International Thoracic Organ Transplant Registry of the International Society for Heart and Lung Transplantation: Thirty-fifth adult lung and heart-lung transplant report-2018; Focus theme: Multiorgan Transplantation. J Heart Lung Transplant 2018;37(10):1169-1183.

3. Singer J.P., Singer L.G. Quality of life in lung transplantation. Semin Respir Crit Care Med 2013;34(3):421-430.

4. Collins S. Nutritional management of cystic fibrosis an update for the 21st century. Paediatr Respir Rev. 2017.

5. Schwarzenberg S.J., Hempstead S.E., McDonald C.M., Powers S.W., Wooldridge J., Blair S., et al. Enteral tube feeding for individuals with cystic fibrosis: Cystic Fibrosis Foundation evidenceinformed guidelines. J. Cyst. Fibros. 2016;15(6):724-735.

6. Sullivan J.S., Mascarenhas M.R. Nutrition: prevention and management of nutritional failure in Cystic Fibrosis. J. Cyst. Fibros. 2017;16(Suppl 2):S87-S93.

7. Hollander F.M., de Roos N.M., Heijerman H.G.M. The optimal approach to nutrition and cystic fibrosis: latest evidence and recommendations. Curr Opin Pulm Med. 2017;23(6):556-61.

8. Turck D., Braegger C.P., Colombo C., Declercq D., Morton A., Pancheva R., et al. ESPEN-ESPGHANECFS guidelines on nutrition care for infants, children, and adults with cystic fibrosis. Clin Nutr 2016;35(3):557-577.

9. Hirche T.O., Knoop C., Hebestreit H., Shimmin D., Sole A., Elborn J.S., et al. Practical guidelines: lung transplantation in patients with cystic fibrosis. Pulm Med 2014;2014:621342.

10. Jomphe V., Lands L.C. and Mailhot G. Nutritional Requirements of Lung Transplant Recipients: Challenges and Considerations. Nutrients. 2018;10(6).

11. Moudiou T., Galli-Tsinopoulou A., Vamvakoudis E., Nousia-Arvanitakis S. Resting energy expenditure in cystic fibrosis as an indicator of disease severity. J Cyst Fibros. 2007;6(2):131-136.

12. Oshima T., Berger M.M., De Waele E., Guttormsen A.B., Heidegger C.P., Hiesmayr M., et al. Indirect calorimetry in nutritional therapy. A position paper by the ICALIC study group. Clin Nutr. 2017;36(3):651-662.

13. Harris J.A., Benedict F.G. A Biometric Study of Human Basal Metabolism. Proc Natl Acad Sci U S A 1918;4(12):370-373.

14. Roza A.M., Shizgal H.M. The Harris Benedict equation reevaluated: resting energy requirements and the body cell mass. Am J Clin Nutr 1984;40(1):168-82.

15. Schofield W.N. Predicting basal metabolic rate, new standards and review of previous work. Hum Nutr Clin Nutr. 1985;39(Suppl 1): 5-41.

16. Energy and protein requirements. Report of a joint FAO/WHO/UNU expert consultation. World Health Organ Tech Rep Ser. 1985;724:1-206. 
17. Weijs P.J., Kruizenga H.M., van Dijk A.E., van der Meij B.S., Langius J.A., Knol D.L., et al. Validation of predictive equations for resting energy expenditure in adult outpatients and inpatients. Clin Nutr. 2008;27(1):150-157.

18. Hollander F.M., Kok A., de Roos N.M., Belle-van Meerkerk G., van de Graaf E.A. Prediction equations underestimate resting energy expenditure in patients with end-stage cystic fibrosis. Nutr Clin Pract. 2017;32(1):116-21.

19. Kalnins D., Pencharz P.B., Grasemann H., Solomon M. Energy expenditure and nutritional status in pediatric patients before and after lung transplantation. J Pediatr 2013;163(5):1500-1502.

20. Dorlochter L., Roksund O., Helgheim V., Rosendahl K, Fluge G. Resting energy expenditure and lung disease in cystic fibrosis. J Cystic Fibros 2002;1(3):131-136.

21. Elborn J.S., Cordon S.M., Western P.J., Macdonald I.A., Shale D.J. Tumour necrosis factor-alpha, resting energy expenditure and cachexia in cystic fibrosis. Clin Sci 1993;85(5):563-568.

22. Singer J.P., Peterson E.R., Snyder M.E., Katz P.P., Golden J.A., D’Ovidio F., et al. Body composition and mortality after adult lung transplantation in the United States. Am J Respir Crit Care Med 2014;190(9):1012-1021.

23. Hollander F.M., van Pierre D.D., de Roos N.M., van de Graaf E.A., lestra J.A. Effects of nutritional status and dietetic interventions on survival in Cystic Fibrosis patients before and after lung transplantation. J Cystic Fibros 2014;13(2): 212-218.

24. Braun A.T., Dasenbrook E.C., Shah A.S., Orens J.B., Merlo C.A. Impact of lung allocation score on survival in cystic fibrosis lung transplant recipients. J Heart Lung Transplant 2015;34(11):1436-1441.

25. Frankenfield D., Roth-Yousey L., Compher C. Comparison of predictive equations for resting metabolic rate in healthy nonobese and obese adults: a systematic review. J Am Diet Assoc. 2005;105(5):775-89.

26. Obesity: preventing and managing the global epidemic. Report of a WHO consultation. World Health Organ Tech Rep Ser. 2000; 894: i-xii, 1-253.

27. Schutz Y., Kyle U.U., Pichard C. Fat-free mass index and fat mass index percentiles in Caucasians aged 18-98 y. Int J Obes Relat Metab Disord 2002;26(7):953-960.

28. Quanjer P.H., Stanojevic S., Cole T.J., Baur X., Hall G.L., Culver B.H., et al. Multi-ethnic reference values for spirometry for the 3-95-yr age range: the global lung function 2012 equations. Eur Respir J 2012;40(16):1324-43.

29. Borowitz D., Lubarsky B., Wilschanski M., Munck A., Gelfond D., Bodewes F., et al. Nutritional status improved in cystic fibrosis patients with the G551D mutation after treatment with ivacaftor. Dig Dis Sci 2016;61(1):198-207.

30. Stallings V .A., Sainath N., Oberle M., Bertolaso C., Schall J.I. Energy Balance and Mechanisms of Weight Gain with Ivacaftor Treatment of Cystic Fibrosis Gating Mutations. J Pediatr. 2018; 201:22937 e4.

31. Ward S.A., Tomezsko J.L., Holsclaw D.S., Paolone A.M. Energy expenditure and substrate utilization in adults with cystic fibrosis and diabetes mellitus. Am J Clin Nutr. 1999;69(5):913-919.

32. Beghin L., Gottrand F., Michaud L., Loeuille G.A., Wizla-Derambure N., Sardet A., et al. Impact of intravenous antibiotic therapy on total daily energy expenditure and physical activity in cystic fibrosis children with Pseudomonas aeruginosa pulmonary exacerbation. Pediatr Res 2003;54(5):756-761. 
Chapter 5

33. Steinkamp G., Drommer A., von der Hardt H. Resting energy expenditure before and after treatment for Pseudomonas aeruginosa infection in patients with cystic fibrosis. Am J Clin Nutr 1993;57(5):685-689.

34. Schwebel C., Pin I., Barnoud D., Devouassoux G., Brichon P.Y ., Chaffanjon P., et al. Prevalence and consequences of nutritional depletion in lung transplant candidates. Eur Respir J. 2000;16(6):1050-5. 
Decreased REE after LTX improves energy prediction 
蛋

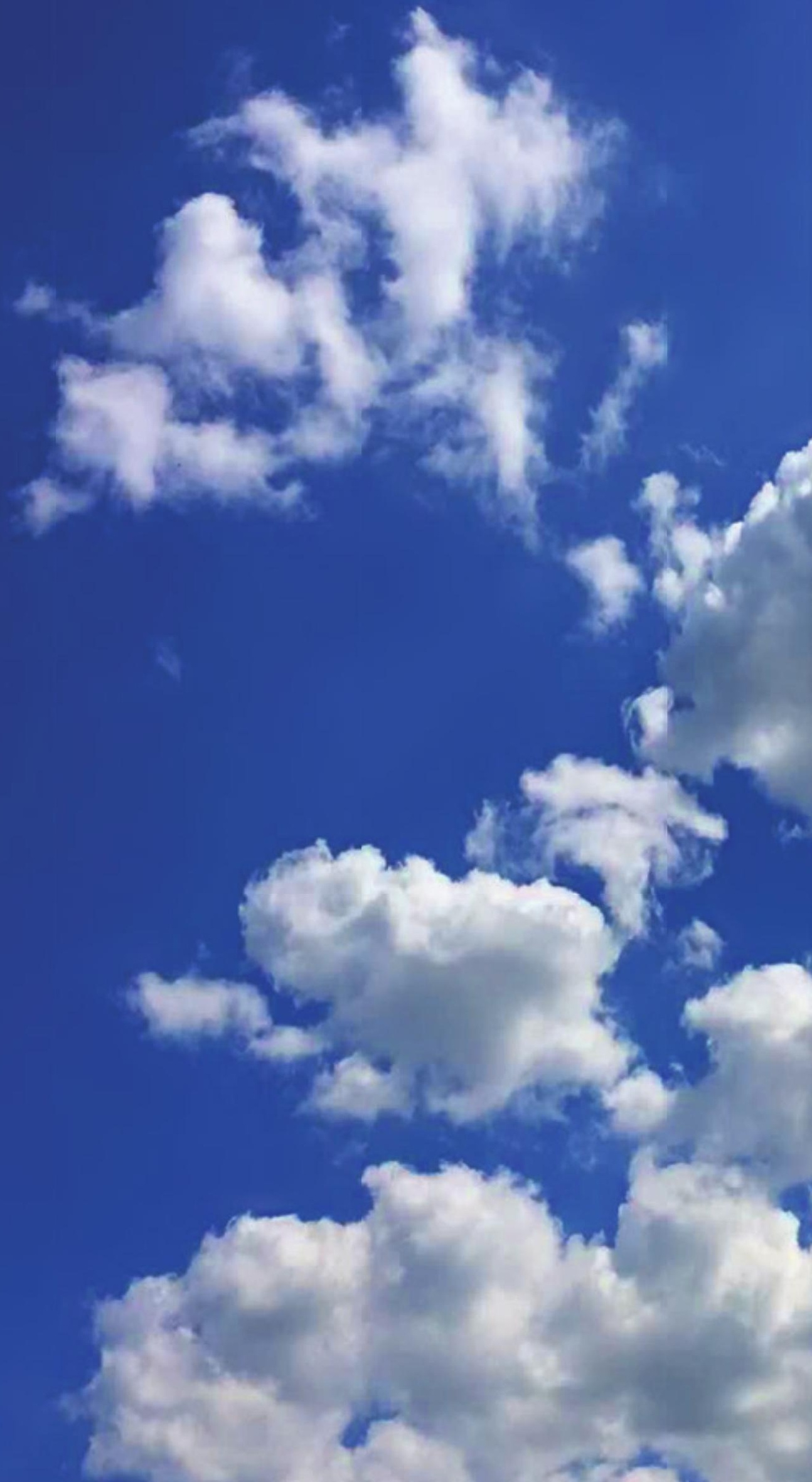




\title{
Chapter 6
}

\section{BODY WEIGHT AND BODY MASS INDEX IN PATIENTS WITH END-STAGE CYSTIC FIBROSIS STABILIZE AFTER THE START OF ENTERAL TUBE FEEDING}

\author{
Francis M. Hollander \\ Nicole M. de Roos \\ Gerdien Belle van Meerkerk \\ Ferdinand Teding van Berkhout \\ Harry G.M. Heijerman \\ Ed A. van de Graaf
}

Journal of the Academy of Nutrition and Dietetics 2017 Nov;117(11):1808-1815 


\section{ABSTRACT}

\section{Background}

Enteral tube feeding (ETF) is widely used in patients with cystic fibrosis (CF) and end-stage lung disease, but previous studies have been limited to investigating whether ETF improves outcomes in patients with moderately or mildly impaired pulmonary function.

\section{Objective}

This study investigated body weight, body mass index (BMl; calculated as $\mathrm{kg} / \mathrm{m}^{2}$ ), pulmonary function, and the presence of CF-related diabetes before and after the start of ETF.

\section{Design}

This was a retrospective observational study.

\section{Participants/setting}

Data from 26 adult patients in an outpatient setting who had end-stage CF (19 women) and had been using ETF for at least 6 months between 2000 and 2014 were analyzed.

\section{Main outcome measures}

Body weight, BMI, pulmonary function (forced expiratory volume in 1 second as percent of predicted) and incidence of CF-related diabetes from 6 months before to 6 months after starting ETF.

\section{Statistical analyses performed}

Time effects were tested with one-way analysis of variance for data that were normally distributed and the Friedman test for non-parametric data. Correlations were tested with Pearson's $r$ or Spearman's $p$, depending on the distribution of the data.

\section{Results}

Mean body weight increased by $3.5 \mathrm{~kg}(95 \% \mathrm{Cl} 2.2$ to $4.8 \mathrm{~kg})$ after patients started ETF. In women, mean BMI decreased by $0.7 \mathrm{~kg} / \mathrm{m}^{2}$ in the 6 months before the start of ETF $(P<0.05)$ and increased by $1.4 \mathrm{~kg} / \mathrm{m}^{2}$ in the 6 months thereafter $(P<0.05)$. In men, BMI changes were similar (-0.8 and +1.1), but not statistically significant. Forced expiratory volume in 1 second as percent of predicted significantly decreased in time from a median of $28 \%$ to $26 \%$ at the start of ETF to $25 \%$ after 6 months ( $P=0.0013)$, with similar trends in women and men. There was no correlation between changes in weight and lung function. CFRD was already present in twelve patients and developed in one more patient after the start of ETF.

\section{Conclusions}

ETF improved body weight and BMI but not pulmonary function in 26 patients with end-stage CF. Clinical outcomes were similar in women and men, but the sample size of men was too small to determine statistical significance. 


\section{INTRODUCTION}

Cystic fibrosis (CF) is the most common life-shortening autosomal recessive disorder in white populations. ${ }^{1}$ The primary symptoms of CF are exocrine pancreatic insufficiency and progressive pulmonary destruction. ${ }^{1}$ The survival of CF patients is closely related to their nutritional status. ${ }^{2,3}$ According to a report from the European CF Foundation patient registry, 9.5\% of CF patients still experience severe malnutrition, despite the available guidelines for the prevention and correction of nutritional deficiencies in CF. ${ }^{4}$ Malnutrition places CF patients at higher risk of morbidity and mortality. ${ }^{5,6}$

Cystic fibrosis-related diabetes (CFRD) is the most common comorbidity in CF patients, affecting one-third to one-half of adult patients.? The pathophysiology of CFRD is linked to a combination of insulin deficiency and insulin resistance. ${ }^{8}$ Insulin therapy is recommended for the treatment of CFRD. ${ }^{9}$ In addition, CFRD is a major risk factor for early mortality in CF patients because of its adverse effects on nutritional status, lung function, and pulmonary infection. ${ }^{?}$

Weight loss in CF patients is caused by increased energy needs and intestinal losses; patients' resting energy expenditure is increased due to lung damage from recurrent respiratory infections and chronic inflammation, ${ }^{10}$ and intestinal losses are increased due to pancreatic insufficiency. ${ }^{11}$ To compensate for these increased energy needs and losses, a higher caloric intake is required. ${ }^{12,13}$ Consensus guidelines recommend that adults with CF aged older than 18 years consume $110 \%$ to $200 \%$ of the energy requirements of same-age healthy adults to maintain or achieve BMI targets. ${ }^{14,15}$ However, as CF patients experience reduced appetite, gastroesophageal reflux, abdominal pain, and infection-related anorexia, achieving these high caloric requirements is often difficult. ${ }^{16}$

Enteral nutritional support is widely used to improve nutritional status in CF patients. ${ }^{13}$ This type of support includes oral nutritional supplementation and enteral tube feeding (ETF). ETF is considered when oral nutritional support fails to improve BMI or to prevent weight loss. ${ }^{5}$ The cutoffs proposed by the European Society for Clinical Nutrition and Metabolism; the European Society for Paediatric Gastroenterology, Hepatology, and Nutrition; and the European Cystic Fibrosis Society guidelines on nutrition care for CF recommend that clinicians use ETF in adults with CF and persistently low $\mathrm{BMI}<18.5$ (underweight) or continuing weight loss (>5\%). In addition, BMI targets for optimal nutritional status in CF adults are $\geq 22$ for women with CF and $\geq 23$ for men. ${ }^{15}$ In addition, the European Society for Clinical Nutrition 
and Metabolism Consensus Statement includes a clear and simple format for diagnosing malnutrition: $\mathrm{BMI}<18.5$ or weight loss (unintentional) $>10 \%$ regardless of time or $>5 \%$ during the past 3 months combined with $\mathrm{BMI}<20$ if younger than 70 years of age. ${ }^{17}$ ETF can be administered by nasogastric, nasoduodenum, percutaneous endoscopic gastrostomy (PEG), or percutaneous endoscopic jejunostomy tube, ${ }^{12}$ depending on the expected duration of nutritional support, the physical and gastrointestinal tract condition, and/or patient preference and choice. Percutaneous radiological gastrostomy is an alternative method for patients ${ }^{18}$ with end-stage lung disease.

Previous studies have been limited to CF patients with moderately or mildly impaired pulmonary function. Two systematic reviews that examined studies of CF patients with moderately or mildly impaired pulmonary function reported positive effects of ETF on nutritional status, ${ }^{13,14}$ and improvements in nutritional status were associated with a higher percentage of CF patients surviving for 2 years or more. ${ }^{14,19}$ One recent study compared changes in lung function, weight gain, and achievement of normal BMI in 17 adult CF patients who started ETF with the corresponding values of 6 patients who required but declined ETF. Two patients in the ETF group died within 1 year because of a decrease in lung function, with a forced expiratory volume in 1 second as percent of predicted (FEV1\%pred) $<28 \%$, but all others showed improved body weight, BMI, and pulmonary function. These effects were not observed in the control group. ${ }^{20}$ However, that study was restricted to CF patients with moderately or mildly impaired pulmonary function, and it is unclear whether the same beneficial effects would be seen in patients with severe lung function impairment. The effectiveness of ETF in improving nutritional status and survival in CF patients with end-stage lung disease has not been investigated. ${ }^{21}$

Previous studies suggest that there may be a difference in survival between sexes. ${ }^{22,23}$ In general, women with CF have a shorter life expectancy than men. ${ }^{23}$ In addition, female sex has been reported to increase the negative effects of CFRD, including pulmonary infections and mortality. ${ }^{24,25}$ These negative outcomes might be due to an effect of both female hormones ${ }^{26}$ and diabetes on a proinflammatory state. One study found a difference in clinical outcomes between sexes after patients started ETF: girls had poorer clinical outcomes than boys. ${ }^{27}$ To our knowledge, no studies have investigated the differences between adult men and women with CF. Therefore, this retrospective study aimed to determine the effects of ETF in men and women with CF and end-stage lung disease on body weight, BMI and pulmonary function. CFRD will be described as a descriptive variable only. 


\section{METHODS}

\section{Study Sample}

This study was a retrospective study that used data on body weight and BMI before and after the start of ETF. Although a randomized controlled study with an ETF and a placebo group would be optimal to test whether ETF is beneficial, this design was not deemed ethical, and retrospective data were thus used.

Patient files for 366 CF patients, documented between 2000 and 2014 at the University Medical Center Utrecht, were available for examination. The following inclusion criteria were used to select the study population: CF patient aged 18 years or older at the start of ETF, start of ETF between January 2000 and June 2014, administration of ETF for at least 6 months, and characteristics of end-stage CF. Patients were used as their own control by comparing their data from before using ETF with data from after starting ETF. CF patients using ETF after lung transplantation were excluded. A waiver of consent was obtained by the Medical Ethics Committee of University Medical Center Utrecht, registered by METC13-605/C.

\section{Study Procedures}

The start of ETF was defined as baseline. Age, sex, genetic mutation, respiratory colonization, route of ETF administration, daily energy requirement as estimated by Schofield's equation, ${ }^{11,28}$ exocrine pancreatic insufficiency, and CFRD were recorded at baseline.

During a dietetic consultation, patients' nutritional status, dietary requirements, and dietary intake (from a dietary history) were assessed. Percentage energy intake by ETF was expressed as the percentage of total energy needs (percent energy requirement). Resting metabolic rate was estimated using Schofield's prediction equation. ${ }^{28}$ Energy requirement was estimated by multiplying resting metabolic rate by activity, disease, and growth factors, and dividing the resulting value by the fat absorption coefficient as follows: (resting metabolic rate $\times$ (activity factor + disease factor -1 ) $\times$ growth factor) / (absorption coefficient). This method was applied consistently across the study sample and has been described in a previous study. ${ }^{21}$ Data on nutritional and clinical outcomes, including weight, height, BMI, and pulmonary function (FEV1\% predicted), were collected during usual outpatient clinic visits at different time points from 6 months before to 6 months after starting ETF. Each subject had to have one to two measurements available for the 6-month time period before and after the start of ETF. When two measurements were available, the mean was used. 


\section{Clinical Assessment Methods}

Body weight was measured on a digital weighing scale (SECA 910; Seca) to the nearest $0.5 \mathrm{~kg}$ with participants barefoot and dressed in indoor clothing. Height was measured in centimeters on a stadiometer (SECA 225; Seca) to the nearest millimeter, and BMI was calculated as weight in kilograms divided by height in meters squared. The adequate BMI targets for patients with CF were as follows: $\mathrm{BMI} \geq 22$ for women and $\geq 23$ for men. ${ }^{15}$ In addition, the main treatment goal of the nutritional team at the University Medical Center Utrecht was to maintain or achieve a normal BMI (18.5 to 24.9) in adult CF patients, especially those with end-stage CF before lung transplantation.

Pulmonary function was assessed by spirometry (Zan). Forced expiratory volume in one second (FEV1 ${ }^{29}$ as a percentage of the FEV1 of a healthy reference population ${ }^{30}$ resulted in the FEV1\% predicted. End-stage CF was defined as having a low pulmonary function, which was determined by a FEV1\%predicted $<30 \%$ and/or a life expectancy $<2$ years. ${ }^{31}$ Presence of CFRD, diagnosed with an oral glucose tolerance test, was extracted from medical records. ${ }^{9}$

Reasons for discontinuation of ETF and mortality within 6 months after the start of ETF and during a follow-up of 4 years after starting ETF were reviewed. Causes of death were defined as end-stage lung disease or respiratory failure, sepsis, multiple organ failure, euthanasia or not recorded. ${ }^{32}$

\section{Statistical Analyses}

All analyses were performed using SPSS ${ }^{33}$ and GraphPad Prism 5 software for Windows ${ }^{34}$; the latter was also used to make graphs. $P$ values $<0.05$ were considered statistically significant. Analyses were performed for the whole group and for men and women separately. Values were reported as the mean \pm standard deviations or medians and interquartile range, depending on the distribution of the data (Shapiro-Wilk's normality test). Time effects were tested with one-way analysis of variance for data that followed a normal distribution, followed by Tukey's multiple comparisons test for differences between time periods; non-parametric data were tested with the Friedman test and Dunn's multiple comparison test. Correlations were tested with Pearson's $r$ or Spearman's p, depending on the distribution of the data. Our study was not powered to test for differences in mortality between men and women or for the influence of CFRD on mortality. 


\section{RESULTS}

Of the 366 CF patients treated at the University Medical Center Utrecht between 2000 and 2014, 85 (23\%) received ETF. Twenty-six patients (19 women; 73\%) met the inclusion criteria and had follow-up data available for analysis (Table 1). FEV1\% predicted was measured before starting ETF in all but one patient, who had pneumothorax, and was available for all three time points for 23 patients. Approximately half of the patients (46\%) had a homozygote $\Delta F 508$ genotype mutation, and $85 \%$ exhibited respiratory colonization with Pseudomonas aeruginosa. At baseline, half of the men and women received ETF by nasogastric tubes (Table 1), which was more common (57.7\%) than starting with PEG tubes (30.8\%).

However, $53 \%$ of the patients who received ETF by nasogastric tubes at baseline switched to gastrostomy feeding tubes $(n=8)$ due to problems with the nasogastric tube, specifically coughing $(n=2)$, deterioration in physical condition $(n=3)$, or expectations to use ETF for more than 6 months $(n=3)$. More women (6 of 19) than men ( 1 of 7$)$ received total ETF $(100 \%$ of energy requirement) at any time point. ETF was used as a nocturnal supplement to oral food intake by 12 of 19 women and 5 of 7 men; as a bolus during the day by 1 of 19 women; for total feeding during a 24-hour period in 6 of 19 women and 1 of 7 men; and continuously throughout the day by 1 of 7 men.

ETF provided two-thirds of energy needs, on average, with a minimum of $33 \%$ and a maximum of $100 \%$. The ETF provided mainly differed in energy and fiber content: 1 patient used a concentrated formula ( $2 \mathrm{kcal} / \mathrm{mL}), 20$ patients used a high-calorie formula (1.5 kcal $/ \mathrm{mL})$, and 5 used a standard caloric formula $(1.0 \mathrm{kcal} / \mathrm{mL})$. Of the 26 patients, 16 used ETF supplemented with multifiber.

At baseline, 14 of 19 women and 6 of 7 men were underweight, based on $\mathrm{BMI}<18.5$. In addition, 18 of 19 women and all 7 men had BMI values below the optimal BMI of $\geq 22 \mathrm{~kg} / \mathrm{m}^{2}$ for women with CF and $\geq 23 \mathrm{~kg} / \mathrm{m}^{2}$ for men (Table 1). Overall, the mean changes in body weight ( $+3.5 \mathrm{~kg}$, $95 \% \mathrm{Cl} 2.2$ to $4.8 \mathrm{~kg})$ and $\mathrm{BMI}(+1.3,95 \% \mathrm{Cl} 0.9$ to 1.8$)$ during the 6 months after starting ETF were significant for the entire group of patients. Before starting ETF, patients' body weight had decreased by $1.9 \mathrm{~kg}(95 \% \mathrm{Cl}-3.0$ to $0.66 \mathrm{~kg}$ ) and BMI by 0.7 ( $95 \% \mathrm{Cl}-1.1$ to 0.3 ).

Eleven women reached BMI values $>18.5$, of which two women reached an optimal BMI value >22: 1 woman had a BMI slightly above 22 at the first two time points, while another woman progressed from a BMI just below 22 to a BMI of 23.6 in the 6 months after starting ETF. On 
Table 1. Baseline characteristics of adult patients with cystic fibrosis and end-stage lung disease $(n=26)$ starting enteral tube feeding between 2000 and 2014 in the Netherlands

\begin{tabular}{|c|c|c|c|c|}
\hline Characteristics & Patients $(n=26)$ & Men $(n=7)$ & Women $(n=19)$ & P value \\
\hline & - & - mean $\pm S D^{a}$ & 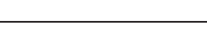 & \\
\hline Age (years) & $28.6 \pm 9.4$ & $32.0 \pm 9.0$ & $27.3 \pm 10.0$ & \\
\hline \multicolumn{5}{|l|}{ Anthropometrics } \\
\hline Weight (kg) & $48.1 \pm 7.2$ & $55.3 \pm 4.7$ & $46.8 \pm 6.6^{*}$ & $<0.05$ \\
\hline $\mathrm{BMI}^{\mathrm{b}}\left(\mathrm{kg} / \mathrm{m}^{2}\right)$ & $17.7 \pm 1.8$ & $17.6 \pm 1.4$ & $17.8 \pm 2.0$ & \\
\hline $\mathrm{BMI}<18.5$ & 20 & 6 & 14 & \\
\hline $\mathrm{BMI} \leq 22$ in women, $\leq 23$ in men & 25 & 7 & 18 & \\
\hline $\mathrm{BMI} \geq 22$ in women & 1 & - & 1 & \\
\hline \multicolumn{5}{|l|}{ Genotype } \\
\hline$\Delta \mathrm{F} 508 / \Delta \mathrm{F} 508$ & $12(46.2)$ & $1(14.3)$ & $11(57.9)$ & \\
\hline$\Delta$ F508/Unknown & $7(26.9)$ & $3(42.9)$ & $4(21.1)$ & \\
\hline$\Delta \mathrm{F} 508 / \mathrm{N} 1303 \mathrm{~K}$ & $1(3.8)$ & $0(0.0)$ & $1(5.3)$ & \\
\hline$\Delta \mathrm{F} 508 / \mathrm{G} 542 \mathrm{X}$ & $1(3.8)$ & $0(0.0)$ & $1(5.3)$ & \\
\hline$\Delta \mathrm{F} 508 / \mathrm{W} 1282 \mathrm{X}$ & $2(7.7)$ & $2(28.6)$ & $0(0.0)$ & \\
\hline$\Delta$ F508/Other & $3(11.5)$ & $1(14.3)$ & $2(10.5)$ & \\
\hline \multicolumn{5}{|l|}{ Respiratory colonization } \\
\hline Pseudomonas aeruginosa & $22(84.6)$ & $6(85.7)$ & $16(84.2)$ & \\
\hline Burkholderia cepacia complex & $3(11.5)$ & $2(28.6)$ & $1(5.3)$ & \\
\hline \multicolumn{5}{|l|}{ Medical } \\
\hline FEV1\%predc & $24.0[20.8-32.5]$ & $22.5[20.0-24.5]$ & 31.0 [22.4-41.0] & \\
\hline $\mathrm{Pl}^{\mathrm{d}}$ & $24(92.3)$ & $7(100.0)$ & $17(89.5)$ & \\
\hline CFRD $^{e}$ & $13(50.0)$ & $3(42.9)$ & $10(52.6)$ & \\
\hline \multicolumn{5}{|l|}{ Nutritional } \\
\hline Energy requirement (kcal/day) & $2670 \pm 539.8$ & $3079 \pm 578.7$ & $2520 \pm 486.9 *$ & $<0.05$ \\
\hline $\begin{array}{l}\text { Energy intake by ETF }{ }^{f} \text { (\%energy } \\
\text { requirement) }\end{array}$ & $65 \pm 22.1$ & $57 \pm 20.9$ & $69 \pm 23.8$ & \\
\hline \multicolumn{5}{|l|}{ Route of administration } \\
\hline Nasogastric tube & $15(57.7)$ & $4(57.1)$ & $11(57.9)$ & \\
\hline PEGh & $8(30.8)$ & $2(28.6)$ & $6(31.6)$ & \\
\hline PRGi & $2(7.7)$ & - & $2(10.5)$ & \\
\hline$P E J^{j}$ & - & - & - & \\
\hline Nasoduodenum tube & $1(3.8)$ & $1(14.3)$ & - & \\
\hline
\end{tabular}

aSD $=$ Standard Deviation.

${ }^{\mathrm{b}} \mathrm{BMI}=$ Body Mass Index.

'FEV1\% pred=Forced expiratory volume in 1 second as $\%$ of predicted.

dPI=Exocrine Pancreas Insufficiency.

eCFRD=Cystic Fibrosis-related Diabetes.

${ }^{\mathrm{f}} \mathrm{ETF}=$ Enteral Tube Feeding.

hPEG=Percutaneous Endoscopic Gastrostomy.

'PRG=Percutaneous Radiologic Gastrostomy.

jPEJ=Percutaneous Endoscopic Jejunostomy.

*Significantly different from men $(p<0.05)$. 
average, women's BMI significantly decreased by 0.7 in the 6 months before starting ETF $(P<0.05)$, but significantly increased by 1.1 after starting $\operatorname{ETF}(P<0.05)$ to a value that was higher than their pre-ETF value (Figure 1). Mean body weight increased by $3.3 \mathrm{~kg}$ ( $95 \% \mathrm{Cl} 1.7$ to $4.9 \mathrm{~kg}$ ) after the start of ETF. Three men reached BMI values $>18.5$, but none of the men reached the optimal BMI of $\geq 23$ at any time point. Changes in mean BMI over time were not significant due to the small sample size but showed the same clinical patterns as in women: a decrease of 0.7 before the start of ETF followed by an increase of 1.1 (Figure 1). Body weight increased by a mean of $4 \mathrm{~kg}$ after the start of ETF, but this increase was not significant ( $95 \% \mathrm{Cl}-1.2$ to $9.1 \mathrm{~kg})$.

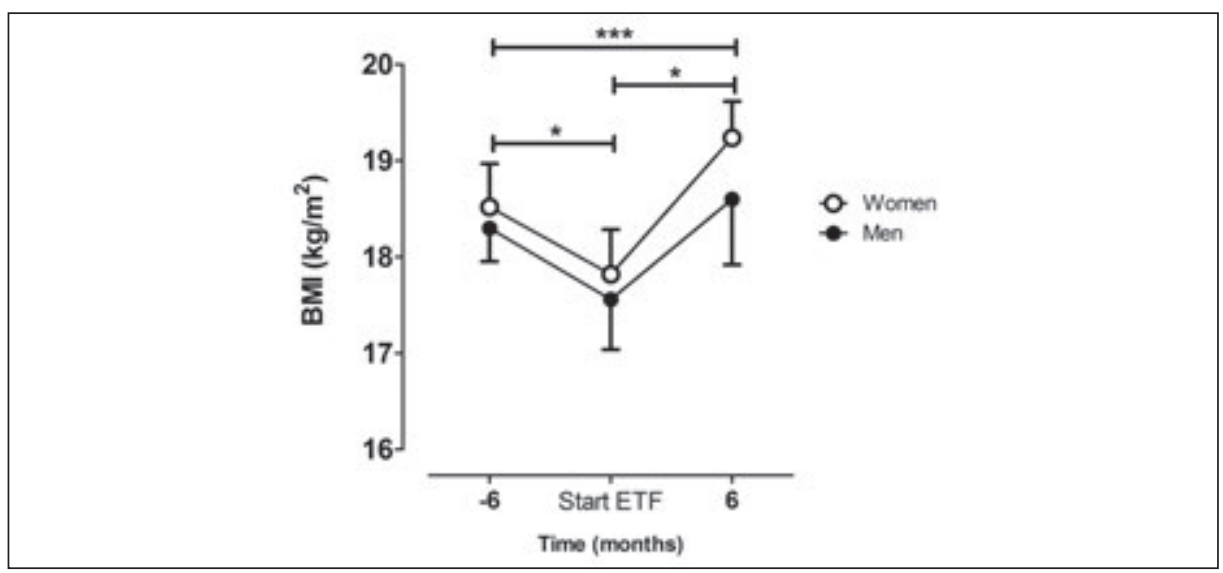

Figure 1. Body Mass Index (BMI) six months before, at the start, and six months after the start of enteral tube feeding in women $(n=19)$ and men $(n=7) .{ }^{* * * *}$ Significant differences between six months before and six months after the start of enteral tube feeding $\left({ }^{*} p<0.05, * * * p<0.001\right)$.

Pulmonary function was low and remained low, with a significant downward trend: FEV1\% predicted was $28 \%$ at 6 months before the start of ETF, $26 \%$ at baseline, and $25 \%$ in the 6 months after the start of ETF $(P=0.0013)$. The same trend was seen in women and in men, although the changes in men were not significant (Table 2). As expected, in patients with end-stage lung disease, no correlation was found between changes in body weight or BMI and lung function in the 6 months after starting ETF (data not shown).

Before starting ETF, CFRD was present in approximately half of the patients (9 women and 3 men). Only one woman was newly diagnosed with CFRD at the start of ETF. After starting $E T F$, no new cases of CFRD occurred. 
Table 2. Body weight and Body Mass Index (means \pm SD) and FEV1\%pred (medians and IQR) for women $(n=19)$ and men $(n=7)$ with cystic fibrosis before and after treatment with enteral tube feeding

\begin{tabular}{|c|c|c|c|c|c|c|}
\hline & \multicolumn{3}{|c|}{ Women $(n=19)$} & \multicolumn{3}{|c|}{ Men $(n=7)$} \\
\hline & 6 mo before & Start ETFa & 6 mo after & 6 mo before & Start ETF & 6 mo after \\
\hline & \multicolumn{6}{|c|}{$\longmapsto$ mean $\pm \mathrm{SD} \longrightarrow$} \\
\hline Body weight (kg) & $48.5 \pm 6.7$ & $47.1 \pm 6.8$ & $50.4 \pm 5.4^{\text {de }}$ & $58.3 \pm 6.1$ & $55.2 \pm 4.7$ & $59.2 \pm 6.2$ \\
\hline $\mathrm{BMI}{ }^{\mathrm{b}}\left(\mathrm{kg} / \mathrm{m}^{2}\right)$ & $18.5 \pm 2.0$ & $17.8 \pm 2.0^{f}$ & $19.2 \pm 1.6^{\text {de }}$ & $18.3 \pm 0.9$ & $17.6 \pm 1.4$ & $18.6 \pm 1.8$ \\
\hline FEV1\%pred & $\begin{array}{c}34 \\
{[25.5-39.8]}\end{array}$ & $\begin{array}{c}31 \\
{[22.5-41.0]}\end{array}$ & $\begin{array}{c}29.5 \\
{[21.8-41.5]^{\mathrm{e}}}\end{array}$ & $\begin{array}{c}22.5 \\
{[21.8-28.1]}\end{array}$ & $\begin{array}{c}22.5 \\
{[20.0-24.5]}\end{array}$ & $\begin{array}{c}21.5 \\
{[18.5-25.4]}\end{array}$ \\
\hline
\end{tabular}

${ }^{\mathrm{a} E T F=e n t e r a l ~ t u b e ~ f e e d i n g . ~}$

${ }^{\mathrm{b}} \mathrm{BMI}=$ body mass index.

'FEV1\%pred=forced expiratory volume in 1 second as \% of predicted.

${ }^{d}$ Significantly $(p<0.05)$ different from 6 months before start of ETF.

eSignificantly $(p<0.05)$ different from start of ETF.

fSignificantly $(p<0.05)$ different from 6 months before start of ETF.

Within 6 months after the start of ETF, a 26 -year-old female patient died of hypercapnia due to end-stage lung disease and a 29-year-old male patient died while on the waiting list for lung transplantation. Both deceased patients were pancreas-insufficient, and the woman also had CFRD. Their data were included in post-lung transplant data. During a follow-up of 4 years after starting ETF, 16 patients (62\%) survived. Of the 10 patients who did not survive, 8 (63\% women) had CFRD. Patients who started ETF remained on ETF for the remainder of their waiting-list time to prevent unwanted weight loss. Cause of death was recorded as respiratory failure in end-stage CF $(n=4)$, lung transplantation-related complication $(n=2)$, sepsis $(n=1)$, multiple organ failure $(n=1)$, euthanasia $(n=1)$, and not recorded $(n=1)$.

\section{DISCUSSION}

In the first 6 months after patients with end-stage CF starting tube feeding, their body weight increased by approximately $3.5 \mathrm{~kg}$, which corresponded to a 1.3 increase in BMI. Lung function, measured as FEV1\% predicted, did not improve. No new cases of CFRD were recorded after the start of ETF. Changes over time were similar in men and women.

In our study sample, $P$ aeruginosa colonization was observed in the majority of patients (85\%). $P$ aeruginosa can lead to increased energy demands ${ }^{35}$ and is associated with lung impairment. ${ }^{36,37}$ The low lung function in our patients may also be influenced by the high 
prevalence of $P$ aeruginosa infection. Pancreas insufficiency did not change before or after ETF use and has been linked to genotype mutation. ${ }^{36}$ As expected, CFRD was highly prevalent in our study population (50\%) and was more pronounced in women than in men, which is in accordance with earlier findings. ${ }^{7}$ With adequate diagnostics and treatment of CFRD, ${ }^{38}$ normalization of blood glucose levels can be maintained during ETF. In our population, 10 of 26 patients (38\%) died in the 4 years after starting ETF; 8 had CFRD, and of those, 5 were women. This finding is in line with previous results. ${ }^{25}$

According to previous and new guidelines for CF care, ETF should be started for unintended weight loss or patients who are underweight. 12,13,15 This deterioration in nutritional status was reflected in our data; both men and women had a median $\mathrm{BMI}<18.5$ and a high percentage of weight loss before starting ETF. Although we cannot fully attribute the improvement in $\mathrm{BMI}$ and body weight to the use of ETF because of the lack of a control group, it seems reasonable that ETF use was effective within 6 months. After the start of ETF, body weight and BMI improved in both men and women, although the improvement was only statistically significant in women. Due to the small sample size, no statistically significant difference was obtained in men. For now, any improvement in body weight that results in a BMI >18.5 seems to be a clinically meaningful outcome of ETF. To make more specific recommendations, longterm follow-up studies are needed that provide estimates for the relation between changes in body weight (BMI) and survival. Until then, ETF should be continued at least until a BMI of 18.5 is reached. The observed increase in weight of $3.5 \mathrm{~kg}$, or the corresponding increase of 1.3 points in BMI, is clinically relevant for CF patients with end-stage lung disease. In the study lung transplant center, a patient with a $\mathrm{BMI}>18.5 \mathrm{~kg} / \mathrm{m}^{2}$ would be included in the transplant program. If patients can achieve this goal using ETF, lung transplantation is possible, with survival as the outcome. If ETF is declined, weight would likely not stabilize but would in fact decline, and patients would thus be excluded from lung transplantation. In addition, if their nutritional status deteriorates, CF patients would be at a higher risk of frequent pulmonary infections and exacerbations with a decrease in quality of life. Improvements in weight and $\mathrm{BMI}$ have been reported within 1 year after starting ETF in adult CF patients ${ }^{20}$ and within 6 months in CF children. ${ }^{39,40}$ In addition, in accordance with previous studies, a small downward trend was observed in pulmonary function..$^{13,14}$

In patients with end-stage pulmonary disease, improving nutritional status may not be sufficient to stop the decrease in pulmonary function, but the data suggest that it could slow this decrease. If this trend is true, improving nutritional status may also increase survival. The median time spent on the waiting list before receiving a donor lung is 15 months (interquartile 
range 6 to 28) in the current study hospital (data not published) and is in concordance with the waiting-list time in the United States. ${ }^{40,41}$ Improved nutritional status contributes to survival while waiting for lung transplantation, which is an important outcome for waiting-list patients.

Outpatient nutritional counseling for ETF patients to monitor nutritional and clinical changes is highly recommended. ${ }^{14,38}$ The current study hospital specializes in both CF and lung transplant care. The prevalence of patients with end-stage lung disease is higher than that in nontransplant CF centers, and this increased prevalence may explain the outcome differences in $\mathrm{BMI}$ and $\mathrm{FEV} 1 \%$ predicted in patients with severely impaired lung function compared to those reported in a study by White and colleagues. ${ }^{20}$ They also reported an improvement in BMI after starting ETF with a duration of at least 3 years. Moreover, in their study, the decrease in lung function, measured as FEV1\% predicted, was reversed. ${ }^{20}$

The route of ETF provision has changed within the past 10 years in the current study hospital. PEG was the more prevalent route between 2005 and 2010, whereas percutaneous radiologic gastrostomy has been more prevalent since 2010. Using PEG ${ }^{19}$ and percutaneous radiologic gastrostomy instead of nasogastric tubes is a treatment option that is less burdensome and is now the preferred route of administration for this vulnerable population in the study CF and lung transplant center.

In this observational study, ETF was categorized as a yes/no variable, regardless of the volume or type of ETF provided. However, it is not uncommon for CF patients with end-stage lung disease to need changes in ETF due to gastroesophageal reflux disease or distal intestinal obstructive syndrome. These complications did occur in the current study population but could not be accounted for in the analysis due to the limited sample size. Furthermore, in other patient groups, the use of ETF in clinical practice can vary regarding the routes of administration, type and quantity. Therefore, larger prospective studies are needed to test whether it is more effective to use total or supplementary ETF to increase body weight in underweight patients and whether differences between caloric and protein content are reflected in differences in effect or tolerability. More research should be conducted to investigate the effects of ETF and mode of ETF on patient's quality of life and nutritional, clinical, and health status. In addition to ETF, other factors influence disease progression such as changes in physical activity, pulmonary infections, frequency of antibiotic treatment, and CFRD treatment. Cystic Fibrosis Foundation registries in the United States, Canada, Europe and Australia can be valuable resources for future studies because they contain data on a large population of CF patients. In addition, a follow-up prospective study design, 
including data on body composition and quality of life changes, may be needed to identifying significant outcomes in both women and men with CF after ETF use. If larger studies confirm the increases in body fat mass, it may be prudent to combine ETF with an exercise program to ensure that more fat-free mass is formed.

There are some limitations regarding the design and outcomes of this retrospective observational study. First, the use of body weight and BMI data only was a limitation; complete data on body composition were lacking. Although clinical measurements of body composition were performed in more than half of the patients selected for lung transplantation in the current study, longitudinal data analyses could not be performed due to missing data. Altered body composition, particularly loss of fat-free mass, in CF patients and its associations with the severity of lung disease, systemic inflammation, resting energy expenditure, catabolic intermediary metabolism, and the rate of exacerbations of respiratory symptoms have been described by Bolton and colleagues ${ }^{42}$ and others. ${ }^{27}$ It would have been helpful to examine whether the increases in body weight in the current study were fat mass or fat-free mass. A small study with six CF patients aged 13 to 25 years old found an increase in body fat mass and no changes in fat-free mass after the start of ETF. ${ }^{43}$ Second, the sample size was small; of the 85 patients who used ETF at any point in time, only 26 patients used it for at least 6 months and had available body weight measurements from 6 months before ETF, at the start of ETF, and 6 months after the start of ETF. Larger studies are needed to test whether the effects of ETF differ for men and women and for other subgroups, for example, groups based on lung function. Third, observational studies such as these cannot be used to determine cause and effect. Although the effects of ETF over time are plausible, other factors, such as use of supplements, medication, changes in treatment guidelines, and physical activity, could also have contributed to the improvements in body weight.

Although this study was performed in only one center in the Netherlands, the results may be generalizable to other centers and other countries: tube feeding formulas are similar in different countries, and the treatment of CF patients is comparable across countries due to international guidelines. However, in low socio-economic countries, CF treatment options may be limited and CF patients remain a vulnerable population with a risk of worse health outcomes. ${ }^{44}$

Newborn screening (NBS) for CF leads to better lung function, nutritional status and improved survival in screened patients in early adulthood. ${ }^{45,46}$ However, NBS was not implemented in the Netherlands until 2011, and thus most adult CF patients were diagnosed at a later stage 
in life. Therefore, patients in the current study may have worse clinical outcomes than those in countries with a longer history of NBS. It is too early to observe the effects of NBS in the current study population.

According to the new European Society for Clinical Nutrition and Metabolism/European Society for Paediatric Gastroenterology, Hepatology, and Nutrition/European Cystic Fibrosis Society guidelines on nutrition care for CF, medical centers are advised to monitor CF patients systematically every 3 to 6 months..$^{15}$ The cutoff points that European Society for Clinical Nutrition and Metabolism recommends for dietitians regarding the use of ETF in patients are Iow BMI values or unwanted weight loss. In our study population, patients were advised to start ETF but had the autonomy to refuse. However, when CF patients want to be included in the lung transplantation program, a minimum BMI of 18.5 is warranted in the study hospital, and ETF is thus started to meet this criterion. New guidelines with higher normal BMI targets for CF have been published since the start of this study. If patients had started ETF earlier, the deterioration in BMI and lung function may have been reduced.

In addition to information on body weight, additional data on gene mutations, pancreatic insufficiency, CFRD, type and frequency of pulmonary infections, physical activity, energy needs, amount, type and mode of ETF, and oral energy intake are needed to obtain a clear picture of energy needs and the effect of treatment. These factors can all be easily stored and extracted from electronic patient databases, but the data need to be accurately and completely entered to ensure their suitability for research. In time, this approach could enable personalized nutritional regimens for adult CF patients specified by age; sex; genetic mutation; and medical, clinical, and nutritional status.

\section{CONCLUSIONS}

ETF improved body weight and BMI but not pulmonary function in 26 patients with end-stage CF. The clinical outcomes were similar in women and men, but the sample size for men alone was too small to determine statistical significance. As randomized trials of ETF are unethical, larger prospective observational studies are needed to further describe the effects of tube feeding. 
Statement of potential conflict of interest No potential conflict of interest was reported by the authors.

Funding/support There is no funding to disclose.

Acknowledgements The author thank the patients for taking part in this study, and master student Britt Broersen for organizing and performing the data collection and assisting in the data analysis. 


\section{REFERENCES}

1. Michl RK, Tabori H, Hentschel J, BeckJF, Mainz JG. Clinical approach to the diagnosis and treatment of cystic fibrosis and CFTR-related disorders. Expert Rev Respir Med 2016:1-10.

2. Stephenson AL, Mannik LA, Walsh S, et al. Longitudinal trends in nutritional status and the relation between lung function and BMI in cystic fibrosis: A population-based cohort study. Am J Clin Nutr. 2013;97(4):872-877.

3. Yen EH, Quinton H, Borowitz D. Better nutritional status in early childhood is associated with improved clinical outcomes and survival in patients with cystic fibrosis. J Pediatr. 2013;162(3):530535 e531.

4. Kerem E, Viviani L, Zolin A, et al. Factors associated with FEV1 decline in cystic fibrosis: Analysis of the ECFS patient registry. Eur Respir J. 2014;43(1):125-133.

5. Schwarzenberg SJ, Hempstead SE, McDonald CM, et al. Enteral tube feeding for individuals with cystic fibrosis: Cystic Fibrosis Foundation evidence-informed guidelines. J Cyst Fibros. 2016;15(6):724-735.

6. Salvatore D, Buzzetti R, Mastella G. Update of literature from cystic fibrosis registries 2012-2015. Part 6: Epidemiology, nutrition and complications. Pediatr Pulmonol. 2017;52(3):390-398.

7. Moran A, Dunitz J, Nathan B, Saeed A, Holme B, Thomas W. Cystic fibrosis-related diabetes: Current trends in prevalence, incidence, and mortality. Diabetes Care. 2009;32(9):1626-1631.

8. Kelly A, Moran A. Update on cystic fibrosis-related diabetes. J Cyst Fibros. 2013;12(4):318-331.

9. Moran A, Brunzell C, Cohen RC, et al. Clinical care guidelines for cystic fibrosis-related diabetes: A position statement of the American Diabetes Association and a clinical practice guideline of the Cystic Fibrosis Foundation, endorsed by the Pediatric Endocrine Society. Diabetes Care. 2010;33(12):2697-2708.

10. Dorlochter L, Roksund O, Helgheim V, Rosendahl K, Fluge G. Resting energy expenditure and lung disease in cystic fibrosis. J Cyst Fibros. 2002;1(3):131-136.

11. Moudiou T, Galli-Tsinopoulou A, Nousia-Arvanitakis S. Effect of exocrine pancreatic function on resting energy expenditure in cystic fibrosis. Acta Paediatr. 2007;96(10):1521-1525.

12. Conway SP, Morton A, Wolfe S. Enteral tube feeding for cystic fibrosis. Cochrane Database Syst Rev. 2008;(2):CD001198.

13. Woestenenk JW, Castelijns SJ, van der Ent CK, Houwen RH. Nutritional intervention in patients with cystic fibrosis: a systematic review. J Cyst Fibros. 2013;12(2):102-115.

14. Stallings VA, Stark LJ, Robinson KA, et al. Evidence-based practice recommendations for nutritionrelated management of children and adults with cystic fibrosis and pancreatic insufficiency: Results of a systematic review. J Am Diet Assoc. 2008;108(5):832-839.

15. Turck D, Braegger CP, Colombo C, et al. ESPEN-ESPGHAN-ECFS guidelines on nutrition care for infants, children, and adults with cystic fibrosis. Clin Nutr. 2016;35(3):557-577.

16. Debray D, Mas E, Munck A, Gerardin M, Clouzeau H. Liver disease, gastrointestinal complications, nutritional management and feeding disorders in pediatric cystic fibrosis. Arch Pediatr. 2016;23(12S):12S15-12S20.

17. Cederholm T, Bosaeus I, Barazzoni R, et al. Diagnostic criteria for malnutrition - An ESPEN Consensus Statement. Clin Nutr. 2015;34(3):335-340. 
18. Perona F, Castellazzi G, De luliis A, Rizzo L. Percutaneous radiologic gastrostomy: A 12-year series. Gut Liver. 2010;4(suppl 1):S44-S49.

19. Ledder O, Oliver MR, Heine RG, Graham J, Volders E, Robinson PJ. Clinical audit results in earlier nutritional intervention in malnourished children with cystic fibrosis with improved outcome. J Paediatr Child Health. 2015;51(10):988-993.

20. White H, Morton AM, Conway SP, Peckham DG. Enteral tube feeding in adults with cystic fibrosis; Patient choice and impact on long term outcomes. J Cyst Fibros. 2013;12(6):616-622.

21. Hollander FM, van Pierre DD, de Roos NM, van de Graaf EA, lestra JA. Effects of nutritional status and dietetic interventions on survival in Cystic Fibrosis patients before and after lung transplantation. J Cyst Fibros. 2014;13(2):212-218.

22. Quintana-Gallego E, Ruiz-Ramos M, Delgado-Pecellin I, Calero C, Soriano JB, Lopez-Campos JL. Mortality from cystic fibrosis in Europe: 1994-2010. Pediatr Pulmonol. 2016;51(2):133-142.

23. Harness-Brumley CL, Elliott AC, Rosenbluth DB, Raghavan D, Jain R. Gender differences in outcomes of patients with cystic fibrosis. J Womens Health (Larchmt). 2014;23(12):1012-1020.

24. Milla CE, Billings J, Moran A. Diabetes is associated with dramatically decreased survival in female but not male subjects with cystic fibrosis. Diabetes Care. 2005;28(9):2141-2144.

25. Lewis C, Blackman SM, Nelson A, et al. Diabetes-related mortality in adults with cystic fibrosis. Role of genotype and sex. Am J Respir Crit Care Med. 2015;191:194-200.

26. Chotirmall SH, Smith SG, Gunaratnam C, et al. Effect of estrogen on pseudomonas mucoidy and exacerbations in cystic fibrosis. N Eng/J Med. 2012;366(21):1978-1986.

27. Oliver MR, Heine RG, Ng CH, Volders E, Olinsky A. Factors affecting clinical outcome in gastrostomyfed children with cystic fibrosis. Pediatr Pulmonol. 2004;37(4):324-329.

28. Schofield WN. Predicting basal metabolic rate, new standards and review of previous work. Hum Nutr Clin Nutr. 1985;39(suppl 1):5-41.

29. Kulich M, Rosenfeld M, Campbell J, et al. Disease-specific reference equations for lung function in patients with cystic fibrosis. Am J Respir Crit Care Med. 2005;172(7):885-891.

30. Quanjer PH, Tammeling GJ, Cotes JE, Pedersen OF, Peslin R, Yernault JC. Lung volumes and forced ventilatory flows. Report Working Party Standardization of Lung Function Tests, European Community for Steedl and Coal. Official Statement of the European Respiratory Society. Eur Respir J. 1993;6(suppl):5-40.

31. Morrell MR, Pilewski JM. Lung transplantation for cystic fibrosis. Clin Chest Med. 2016;37(1):127-138.

32. Martin C, Hamard C, Kanaan R, et al. Causes of death in French cystic fibrosis patients: The need for improvement in transplantation referral strategies! J Cyst Fibros. 2016;15(2):204-212.

33. IBM SPSS Statistics for Windows [computer program]. Version 20.0. Armonk, NY: IBM Corp; 2011. 34. GraphPad Software Prism 5 for Windows [computer program]. La Jolla, CA: GraphPad; 2010.

35. Vinton NE, Padman R, Davis M, Harcke HT. Effects of Pseudomonas colonization on body composition and resting energy expenditure in children with cystic fibrosis. JPEN J Parenter Enteral Nutr. 1999;23(4):233-236.

36. Bonadia LC, de Lima Marson FA, Ribeiro JD, et al. CFTR genotype and clinical outcomes of adult patients carried as cystic fibrosis disease. Gene. 2014;540(2):183-190.

37. Olszowiec-Chlebna M, Koniarek-Maniecka A, Stelmach W, et al. Predictors of deterioration of lung function in Polish children with cystic fibrosis. Arch Med Sci. 2016;12(2):402-407. 
38. Moran A, Pillay K, Becker DJ, Acerini CL. International Society for Pediatric and Adolescent Diabetes. ISPAD Clinical Practice Consensus Guidelines 2014. Management of cystic fibrosis-related diabetes in children and adolescents. Pediatr Diabetes. 2014;15(suppl 20):65-76.

39. Bradley GM, Carson KA, Leonard AR, Mogayzel PJ, Jr., Oliva-Hemker M. Nutritional outcomes following gastrostomy in children with cystic fibrosis. Pediatr Pulmonol. 2012;47(8):743-748.

40. Braun AT, Dasenbrook EC, Shah AS, Orens JB, Merlo CA. Impact of lung allocation score on survival in cystic fibrosis lung transplant recipients. J Heart Lung Transplant. 2015;34(11):1436-1441.

41. Thabut G, Christie JD, Mal H, et al. Survivial benefit of lung transplant for cystic fibrosis since lung allocation score implementation. Am J Respir Crit Care Med. 2013;187(12):1335-1340.

42. Bolton CE, Ionescu AA, Evans WD, Pettit RJ, Shale DJ. Altered tissue distribution in adults with cystic fibrosis. Thorax. 2003;58(10):885-889.

43. Pencharz P, Hill R, Archibald E, Levy L, Newth C. Energy needs and nutritional rehabilitation in undernourished adolescents and young adult patients with cystic fibrosis. J Pediatr Gastroenterol Nutr. 1984;3(suppl 1):S147-S153.

44. Iskrov GG, Stefanov RS, Lopez-Bastida J, et al. Economic burden and health-related quality of life of patients with cystic fibrosis in Bulgaria. Folia Med (Plovdiv). 2015;57:56-64.

45. Dijk FN, McKay K, Barzi F, Gaskin KJ, Fitzgerald DA. Improved survival in cystic fibrosis patients diagnosed by newborn screening compared to a historical cohort from the same centre. Arch Dis Child. 2011;96(12):1118-1123.

46. Farrell PM, White TB, Howenstine MS, et al. Diagnosis of cystic fibrosis in screened populations. J Pediatr. 2017;181S:S33-S44.e32. 
Body weight and BMI after the start of enteral tube feeding 
蛋

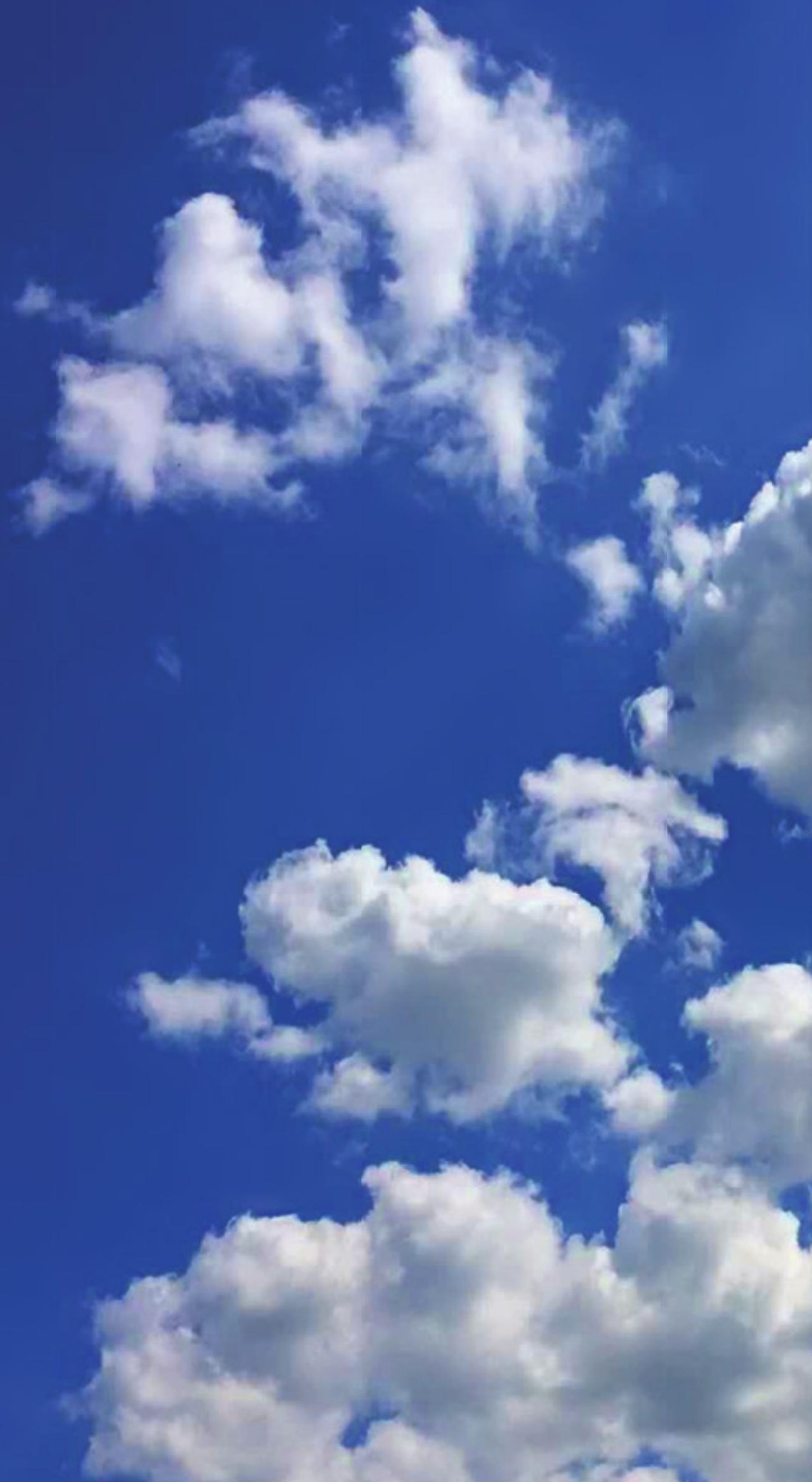




\section{Chapter 7}

\section{EFFECTS OF NUTRITIONAL STATUS AND DIETETIC INTERVENTIONS ON SURVIVAL IN CYSTIC FIBROSIS PATIENTS BEFORE AND AFTER LUNG TRANSPLANTATION}

F.M. Hollander

D.D. van Pierre

N.M. de Roos

E.A. van de Graaf

J.A. lestra

F.M. Hollander and D.D. van Pierre contributed equally to this paper

Journal of Cystic Fibrosis 2014 Mar;13(2):212-218 


\section{ABSTRACT}

\section{Background}

This study retrospectively investigated nutritional status, dietetic intervention and intake in Cystic Fibrosis (CF) patients before and after lung transplantation (LTX).

\section{Methods}

Body mass index (BMI), Fat Free Mass Index (FFMI) and nutritional intake were retrieved from 75 out-patients aged 15-53 years. Patients were seen every 3-4 months during the waiting list time (range 0-81 months) up to 116 months after LTx. Survival was measured in months.

\section{Results}

The median BMI at baseline was $19.2 \mathrm{~kg} / \mathrm{m}^{2}$ (range: 15.3 to $28.4 \mathrm{~kg} / \mathrm{m}^{2}$ ) with 29 patients (39\%) below $\leq 18.5 \mathrm{~kg} / \mathrm{m}^{2}$. FFMI (measured in 65 patients) had a median of $15.2 \mathrm{~kg} / \mathrm{m}^{2}$ (range: 11.1 to $22.4 \mathrm{~kg} / \mathrm{m}^{2}$ ) with 39 patients $(60 \%) \leq 16.7 \mathrm{~kg} / \mathrm{m}^{2}$ (men) or $\leq 14.6 \mathrm{~kg} / \mathrm{m}^{2}$ (women). Median energy intake was $2800 \mathrm{kcal}, 239 \mathrm{kcal}$ higher than the estimated energy requirement. However, 8 patients consumed $\geq 500 \mathrm{kcal}$ less than recommended. Protein intake was 104 (range 60-187) g or $1.9 \mathrm{~g} / \mathrm{kg}$ per day. Despite dietetic intervention with oral nutritional supplements (ONS) (36 patients), tube feeding (12 patients), or both (13 patients), BMI and FFMI hardly improved pre-LTx. LTx was performed in 51 patients (68\%); 10 patients died during follow-up, median survival time was 41 months. A BMI $\leq 18.5 \mathrm{~m}^{2}$ was more prevalent in patients who died before LTx (6/9) or who died after LTx (4/10) than in patients who were still alive on the waiting list (5/15) or who survived LTx (14/41). Results for FFMI were comparable. From 6-12 months postLTX, BMI and FFMI markedly improved, especially in underweight patients.

\section{Conclusion}

A BMI $\leq 18.5 \mathrm{~kg} / \mathrm{m}^{2}$ and an FFMI $\leq 16.7 \mathrm{~kg} / \mathrm{m}^{2}$ (men) or $\leq 14.6 \mathrm{~kg} / \mathrm{m}^{2}$ (women) appears to impair survival in LTx candidates with CF. Patients maintained a low body weight before LTX. After LTX weight gain is achieved. 


\section{INTRODUCTION}

Lung transplantation (LTX) is an established treatment option for end-stage lung disease in Cystic Fibrosis (CF) patients.' An optimal nutritional status pre-and post-lung transplant is an important predictor of survival in CF patients. ${ }^{2,3}$ However, malnutrition is a common problem in patients with CF due to fat malabsorption and a higher energy expenditure as a result of inflammation, frequent periods of severe respiratory tract infections, and increased work of breathing when lung function declines. ${ }^{4,5} \mathrm{~A}$ good indicator of nutritional status in CF patients is Body Mass Index (BMI). ${ }^{2,6}$ Underweight adult CF patients (BMI $<18.5 \mathrm{~kg} / \mathrm{m}^{2}$ ) have a $25 \%$ higher risk of mortality than adult CF patients with a normal weight. ${ }^{7}$ On the other hand, the prevalence of overweight and obesity in CF patients is increasing, with estimates ranging from $10 \%$ in the UK ${ }^{8,9}$ to $18.4 \%$ in Canada. This may not be desirable, because mortality after LTX was shown to be higher in underweight, overweight, and obese patients (including CF patients) than in normal-weight patients. ${ }^{10} \mathrm{~A}$ BMI below $18 \mathrm{~kg} / \mathrm{m}^{2}$ is often used as a preclusion for LTX. ${ }^{1}$ Thus, keeping the BMI between 18 and $30 \mathrm{~kg} / \mathrm{m}^{2}$ before LTX is an important treatment goal.

Lean body mass or fat free mass (FFM) is another indicator for nutritional status. It is a measure of muscle mass and correlates with (inspiratory) muscle function, quality of life, ${ }^{11}$ and lung function. ${ }^{12}$ Depletion of FFM is strongly associated with increased mortality while awaiting LTX and with prolonged post-transplant intensive care unit stays. ${ }^{3}$ This means that not just body weight but also FFM should be monitored in CF patients to avoid fat accumulation and loss of muscle mass.

Pre- and post-transplant nutrition management is aimed to maintain or improve nutritional status, in order to meet the criteria for LTx and optimize survival before and after LTx. Few studies have investigated nutritional intervention strategies in patients on the waiting list for lung transplantation. 2,13,14 One study demonstrated that Percutaneous Endoscopic Gastrostomy (PEG) feeding may improve body weight before LTx in patients with a low BMI. ${ }^{2}$ Another study showed that three appointments with a dietician were not sufficient to improve nutritional status in underweight lung transplant candidates. ${ }^{13}$ An intensive nutritional programme with extra meals and nutritional supplements during hospitalization in underweight patients with end-stage lung disease was effective in increasing energy intake and body weight compared to regular nutritional support. ${ }^{14}$ Until now, insight into the nutritional status and the effect of dietetic intervention in pre- and post-transplant CF patients is limited. Therefore, the aim of this study was to describe nutritional status and 
intake at waiting list entry, subsequent changes in body weight and FFM before and after LTx, and survival after LTx using data from out-patient visits.

\section{METHODS}

\section{Study design}

\section{Data at waiting list entry ( $n=75$ patients)}

In the period August 1998 - June 2011, 110 CF patients from different CF centres in the Netherlands had been screened for LTx. Of these, 98 patients were accepted on the waiting list but we could retrieve data from 75 CF patients (aged $\geq 15$ years) from our own patient files. All LTx transplants were performed in the Lung Transplantation Center, University Medical Center Utrecht.

\section{Follow-up data (31 of 75 patients)}

Of the 75 patients, 51 had an LTx. Unfortunately complete follow-up data on anthropometry and diet were only available for 31 of these patients: 10 died after LTX and 10 patients were followed for less than 1 year. For the remaining 31 patients we retrieved anthropometric data at four time points: (1) waiting list entry (baseline); (2) last outpatient visit pre-LTx; (3) 6-12 months outpatient visit post-LTx and (4) 18-24 months outpatient visit post-LTx. Time between waiting list entry and LTX ranged between 0 and 81 months. Because no nutritional data were available for the moment of LTX, we chose the 'last outpatient visit pre-LTX' for time point 2.

\section{Nutritional management}

Dietetic care was provided by two registered dieticians to maintain or improve nutritional status before and after LTX. During a dietetic consultation patients' nutritional status, dietary requirements, and dietary intake (from a dietary history) were assessed. If necessary, use of additional ONS and/or tube feeding was advised or maintained. Patients were seen as outpatients every 3-4 months. When patients were unable to maintain body weight, the daily use of ONS was increased, tube feeding was started, or the type of tube feeding was changed from normal $(1.5 \mathrm{kcal} / \mathrm{ml})$ to energy enriched $(2 \mathrm{kcal} / \mathrm{ml})$. These adjustments were individual and therefore, they are not reported in this paper. None of the patients used appetite stimulants.

\section{Clinical measurements}

Medical, anthropometric, and nutritional characteristics were determined at baseline and subsequently every 3-4 months when the patient had been admitted to the waiting list. After 
LTx the patients were seen in the outpatient clinic every three months the first year, every six months the second year and once a year after three years.

\section{Medical characteristics}

Patient data and surgery-related characteristics were extracted from electronic medical records.

Pulmonary function was assessed by spirometry (ZAN; Oberthulba, Germany) and expressed as FEV1\% of the predicted normal Forced Expiratory Volume in 1s (FEV1). All FEV1 measurements were performed by the Laboratory for lung function of the UMCU. Cystic Fibrosis-Related Diabetes (CFRD) was recorded because of its negative effect on lung function, particularly in women. ${ }^{15}$ Diagnosis of CFRD was based on the criteria proposed by the American Diabetes Association 2011. ${ }^{16}$

Pancreatic insufficiency was defined as having insufficient pancreatic function to achieve normal intestinal absorption of fat, therefore requiring pancreatic enzyme replacement therapy (PERT) from an early age.

A high urgency $(H U)$ status was assigned to patients whose life expectancy was estimated to be less than three months according to Eurotransplant guidelines. ${ }^{17}$

\section{Anthropometric characteristics}

$B M I$ was classified according to the WHO recommendations ${ }^{18}$ and the European Consensus Report for $\mathrm{CF}^{19}$ : underweight $\leq 18.5 \mathrm{~kg} / \mathrm{m}^{2}$; normal weight $18.5-24.9 \mathrm{~kg} / \mathrm{m}^{2}$ and overweight $25-29.9 \mathrm{~kg} / \mathrm{m}^{2}$. For the 8 adolescents ( $<18$ years) age- and gender-adjusted cut off points for BMI were used as proposed by the Netherlands Organisation for Applied Scientific Research TNO. ${ }^{20}$ Measurements of body weight and height were performed in the Laboratory for lung function of the UMCU while patients were barefoot and dressed in indoor clothing.

Body composition was routinely measured by Bio Impedance Analysis (BIA, Bodystat 1500; Bodystat Ltd, Douglas, UK), while patients were in a fasted state, lying in supine position with arms apart from trunk and legs not touching each other. Measuring body composition by BIA is a part of our lung transplantation protocol and is performed by trained personnel according to standardized methods. BIA is an accepted method for the assessment of FFM ${ }^{21}$ that can be used without modification in Cystic Fibrosis patients. ${ }^{22} \mathrm{~A}$ low Fat Free Mass Index (FFMI) was defined as $\leq 14.6 \mathrm{~kg} / \mathrm{m}^{2}$ for women and $\leq 16.7 \mathrm{~kg} / \mathrm{m}^{2}$ for men. ${ }^{23}$ 
Weight change (\%) was calculated as the changes in body weight at the follow-up time points relative to the initial body weight at baseline.

\section{Nutritional measurements and nutritional requirements}

Resting Metabolic Rate (RMR) was estimated at baseline by the formula of Schofield ${ }^{24}$ that has been in use in our center since 1998. Energy requirement was estimated by multiplying RMR by activity, disease and growth factors and dividing it by the fat absorption coefficient as follows: RMR x (Activity Factor + disease factor - 1) x growth factor / (absorption coefficient). ${ }^{25}$ The Activity Factor was 1.1 for limited active lifestyle, 1.5 for normal active lifestyle and 1.7 for an active lifestyle. The disease factor was set at 1.3 in the pre-LTx period, because all patients had a FEV1 $<40 \% .{ }^{19}$ The growth factor varied from 1.0 (no growth) to 1.04 (puberty growth spurt). For 9 patients, the fat absorption coefficient was based on measurements of fat absorption during a 72-hour faecal fat test. For the 66 patients for whom no data was available, we used an absorption coefficient of 0.93 for pancreatic insufficient patients $(n=64)$ and 0.97 for pancreatic sufficient patients $(n=2)$. These estimates are based on fat digestibility in healthy persons who excrete less than $7 \%$ of the ingested fat with a total daily faecal fat output of less than $7 \mathrm{~g}$ per day. ${ }^{19}$

Protein requirements were estimated as $1.5 \mathrm{~g}$ protein per kg actual body weight, which is the recommendation for undernourished patients. ${ }^{26}$

Energy- and protein intakes were calculated from a dietary history, a face-to-face interview to determine the usual meal pattern, taken at baseline. No intake data were available for the other time points.

\section{Data analyses}

Analyses were performed for the whole group $(n=75)$ and for men and women separately. Because most data were not normally distributed, descriptive statistics were expressed as medians with ranges.

We made the following subgroups: 1. pre-LTX non survivors; 2. pre-LTX survivors; 3. post-LTX non survivors; and 4. post-LTx survivors. To test for differences between subgroups the nonparametric Mann-Whitney $U$ test was used for continuous variables and the Chi-square test for dichotomous variables. 
To test for differences between time points in the follow-up data $(n=31)$, the non-parametric test for dependent variables, the Friedman test, was used. Statistical significance was defined as two-tailed $p$ values less than 0.05 . The data were analysed using the SPSS program for Windows (version 17.0; SPSS Inc, Chicago).

\section{RESULTS}

\section{Waiting list entry $(n=75)$ \\ Study population at baseline}

A total of 75 patients (41 men) were included (Table 1), with a median age of 28 years (range: 15 to 53 years). The median FEV1 was $25 \%$ of predicted (range: 12 to $37 \%$ of predicted). CFRD was diagnosed in $63 \%$ of the patients and was more pronounced in women $(p=0.005)$. Pancreatic insufficiency was diagnosed in all but 2 patients. Of the 75 patients who were admitted to the waiting list for LTx, 51 patients survived long enough to undergo LTX. Seventy-five percent of these patients had a HU status before surgery.

\section{Anthropometrics}

The median BMI at baseline was $19.2 \mathrm{~kg} / \mathrm{m}^{2}$ (range: 15.3 to $28.4 \mathrm{~kg} / \mathrm{m}^{2}$ ) with 29 patients below $\leq 18.5 \mathrm{~kg} / \mathrm{m}^{2}, 45$ patients between 18.5 and $24.9 \mathrm{~kg} / \mathrm{m}^{2}$ and 1 patient with a BMI $\geq 25 \mathrm{~kg} / \mathrm{m}^{2}$. FFMI measurements were available for 65 patients. Their median FFMI was $15.2 \mathrm{~kg} / \mathrm{m}^{2}$ (range: 11.1 to $22.4 \mathrm{~kg} / \mathrm{m}^{2}$ ) with $24 \mathrm{men} \leq 16.7 \mathrm{~kg} / \mathrm{m}^{2}$ and 15 women $\leq 14.6 \mathrm{~kg} / \mathrm{m}^{2}$. Undernourishment based on BMI was 39\% (29/75) against 60\% (39/65) based on FFMI. All patients with a low BMI also had a low FFMI but a 'normal' BMI was almost equally associated with a low or normal FFMI (Figure 1). 
Table 1. Baseline characteristics of CF transplant candidates at UMCU ( $n=75)$.

\begin{tabular}{ll}
\hline Variables & Data \\
\hline Descriptives & \\
Gender, n & 41 men, 34 women \\
Cystic Fibrosis-Related Diabetes, n (\%) & $47(63 \%)$ \\
Pancreatic insufficiency, n (\%) & $73(97 \%)$ \\
Measurements & Median (range) \\
Age, years & $28(15-53)$ \\
Weight, kg & $54.4(40-93)$ \\
Height, m & $1.69(1.41-1.89)$ \\
BMI, kg/m² & $19.2(15.3-28.4)$ \\
FFMI, kg/m² measured in 65 patients & $15.2(11.1-22.4)$ \\
FEV1, \% predicted & $24.5(12-37)$ \\
\hline
\end{tabular}

FEV1 = Forced Expiratory Volume in 1 Second; BMI = Body Mass Index; FFMI = Fat Free Mass Index.

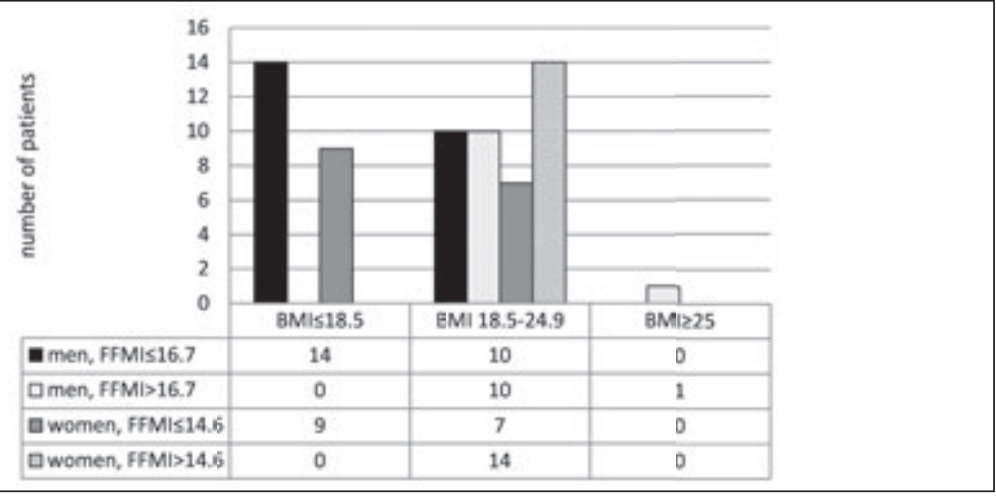

Figure 1. Number of patients with low or normal Fat Free Mass Index (FFMI) according to Body Mass Index (BMI) groups (underweight, normal and overweight). The table includes 65 patients because FFMI data were missing in 10 patients.

\section{Nutritional requirements, intake and interventions}

The median energy intake estimated from the dietary history at baseline was $2800 \mathrm{kcal}$ (range 1570-4700). This was close to the estimated median energy requirement of $2600 \mathrm{kcal}$. However, 8/75 patients had an intake of $500 \mathrm{kcal}$ or more below recommendation. 
Protein requirements were easier to meet; only $19 \%$ of the men and $20 \%$ of the women did not meet the protein requirements of $1.5 \mathrm{~g} / \mathrm{kg} / \mathrm{day}$. Men and women were not different in achieving energy and protein requirements (Table 2 ).

For 74 patients we had information on the use of nutritional supplements at screening (missing data for 1 patient who was screened in another hospital). Of these, 63 patients (85\%) used ONS and/or tube feeding in addition to their diet. For 6 men and 6 women the nutritional intervention was aimed at increasing rather than preserving intake. In these patients, mean weight increase during waiting list time was $2.2 \mathrm{~kg}$. Weight increase in patients who were advised to maintain body weight was $2.8 \mathrm{~kg}$ (difference not significant).Tube feeding (17 out of 25 patients) was mainly through Percutaneous Endoscopic Gastrostomy (PEG) feeding tubes. Energy intake as a percentage of energy requirement was $116 \%$ for patients with a screening $\mathrm{BMI} \leq 18.5$ and $102 \%$ for patients with a BMI $>18.5(p=0.051)$.

Table 2. Nutritional requirements and intake (means and ranges) of 74 CF patients at waiting list entry (1 patient was screened in another hospital, data unavailable).

\begin{tabular}{lll}
\hline & Men $(\mathbf{n}=\mathbf{4 0})$ & Women $(\mathbf{n}=\mathbf{3 4})$ \\
\hline Nutritional requirements & & $1280(1110-1460)$ \\
Estimated Resting Metabolic Rate (kcal/day) & $1600(1320-1960)$ & $2410(1740-3240)$ \\
Energy requirement, kcal/day & $3100(2160-4050)$ & $78(60-110)$ \\
Protein requirement, g/day & $92(62-140)$ & \\
Nutritional intake ${ }^{\alpha}$ & & $101(63-164)(n=33)$ \\
Energy intake (\%energy requirement) & $101(55-160)(n=38)$ & $130(80-220)(n=31)$ \\
Protein intake (\%protein requirement) & $130(73-253)(n=33)$ & \\
\hline
\end{tabular}

a Intake data not complete for all 74 patients.

\section{Baseline nutritional status and survival}

We investigated whether a low BMI or FFMI at waiting list entry was predictive of survival. From those who died before transplantation $(n=9), 67 \%(n=6)$ had a BMI $\leq 18.5 \mathrm{~kg} / \mathrm{m}^{2}$ and $78 \%$ $(\mathrm{n}=7)$ had a low baseline FFMI (men $\leq 16.7$ and women $\leq 14.6 \mathrm{~kg} / \mathrm{m}^{2}$ ). From those who died after transplantation $(n=10), 40 \%(n=4)$ had a low BMI and $60 \%(n=6)$ had a low baseline FFMI. Patients who survived transplantation $(n=41)$ had a somewhat better nutritional status: $34 \%$ $(\mathrm{n}=14)$ had a BMI $\leq 18.5 \mathrm{~kg} / \mathrm{m}^{2}$ and $40 \%(\mathrm{n}=16)$ had a low FFMI (Figure 2). However, medians 
of BMI and FFMI were not significantly different between the 41 LTX survivors and the 10 LTX non-survivors (data not shown).

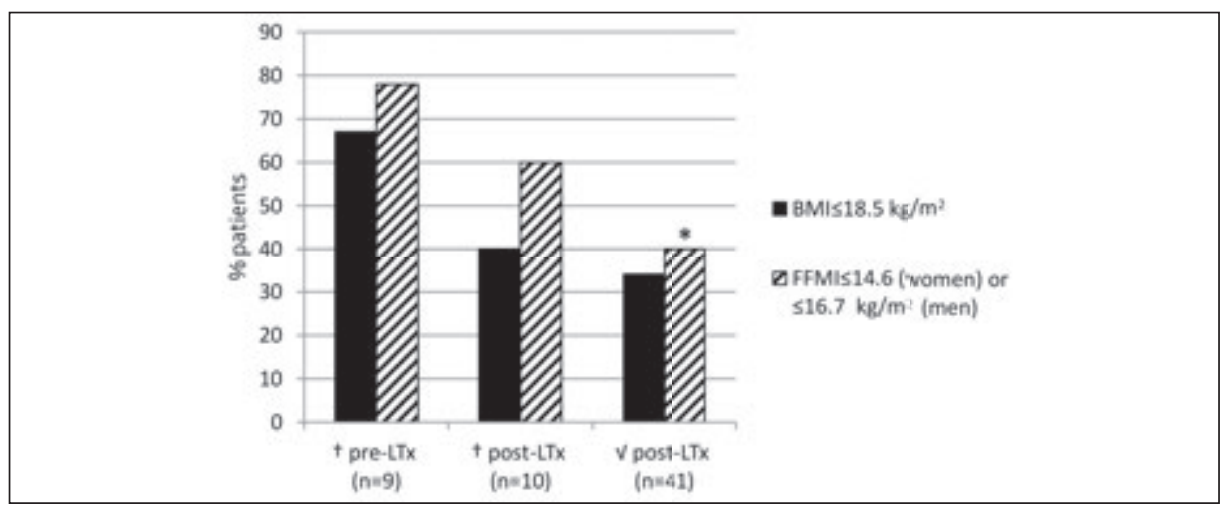

Figure 2. Percentage of patients with a low Body Mass Index (BMI) or low Fat Free Mass Index (FFMI) according to LTx status: †pre-LTx = death before LTx, †post-LTx= death after LTx (within 24 months), $\checkmark$ post-LTX $=$ survived after LTX. Asterisk (*) indicates significant difference from tpre-LTx group. Not in the figure: 15 patients who were still waiting for LTx (making a total of 75 patients).

\section{Follow up data on nutritional status after LTx $(n=31)$ \\ Study population}

Of the 51/75 patients who underwent LTX, follow-up data about nutritional status was available for 31 patients (Figure 3). We were unable to retrieve follow up data for 20 other patients: 10 had died within a year after LTx and 10 had a follow up of less than one year after LTX. These patients were excluded from the data analysis on nutritional status. 


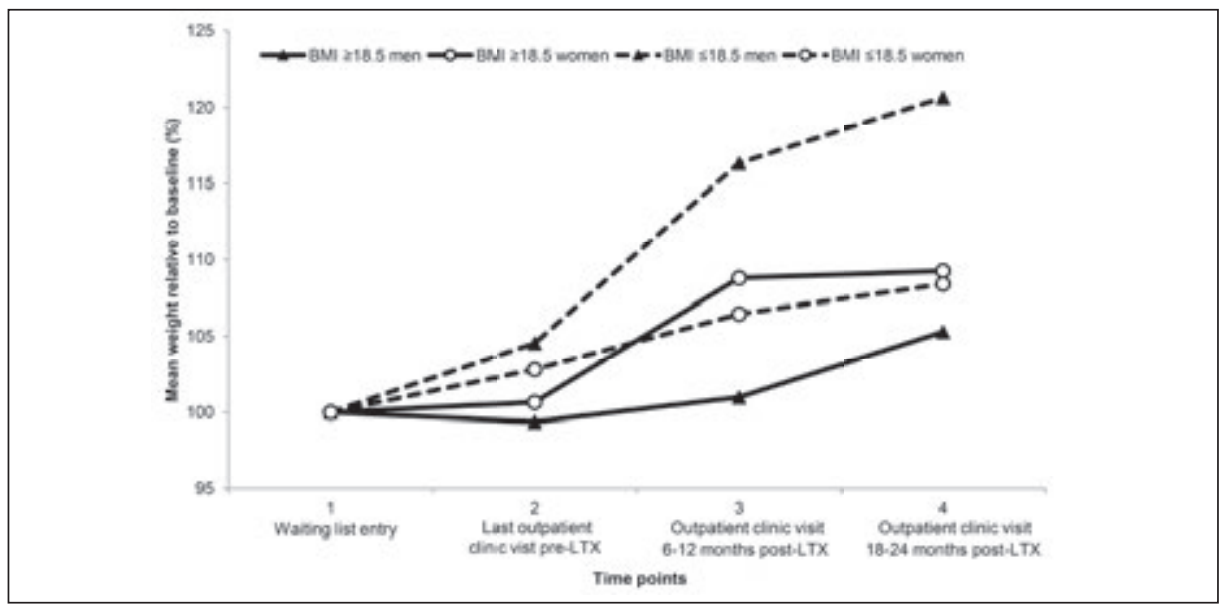

Figure 3. Pre- and post-LTX mean weight expressed as percentage from baseline in men and women with low or normal BMI at four time points (total $n=31$ ).

\section{Anthropometrics}

There was no significant improvement in BMI or FFMI between waiting list entry and the last visit before transplantation, although the number of patients with a $\mathrm{BMI} \leq 18.5 \mathrm{~kg} / \mathrm{m}^{2}$ reduced from $13(42 \%)$ to $8(26 \%)(p=0.025)$ (Table 3$)$. After LTX, BMI and FFMI increased significantly both for men and women compared to the data at baseline (Table 3). Body weight after LTX increased significantly compared to body weight pre-LTX. The mean weight gain at 18-24 months post-LTX, as a proportion of initial body weight, was $11 \%$ for men and $8 \%$ for women (respectively $p=0.001$ and $p=0.034$ ). Strongest increases in body weight were seen in men who started with underweight (Figure 3): at 6-12 and 18-24 months post-LTx this difference between men was statistically significant ( $p=0.001$ and $p=0.015$ ). For women weight gain during follow-up was less affected by initial BMI.

\section{Nutritional interventions}

The type of feeding was known for 20 of the 22 men and 8 of the 9 women. In men, the use of ONS and/or tube feeding decreased significantly during follow up from 17 to 9 ( $p=0.001$ ). In women this number declined from 8 to $3(p=0.025)$. Although overall nutritional status improved, PEG feeding was still necessary in 5 patients at 18-24 months after LTX. 


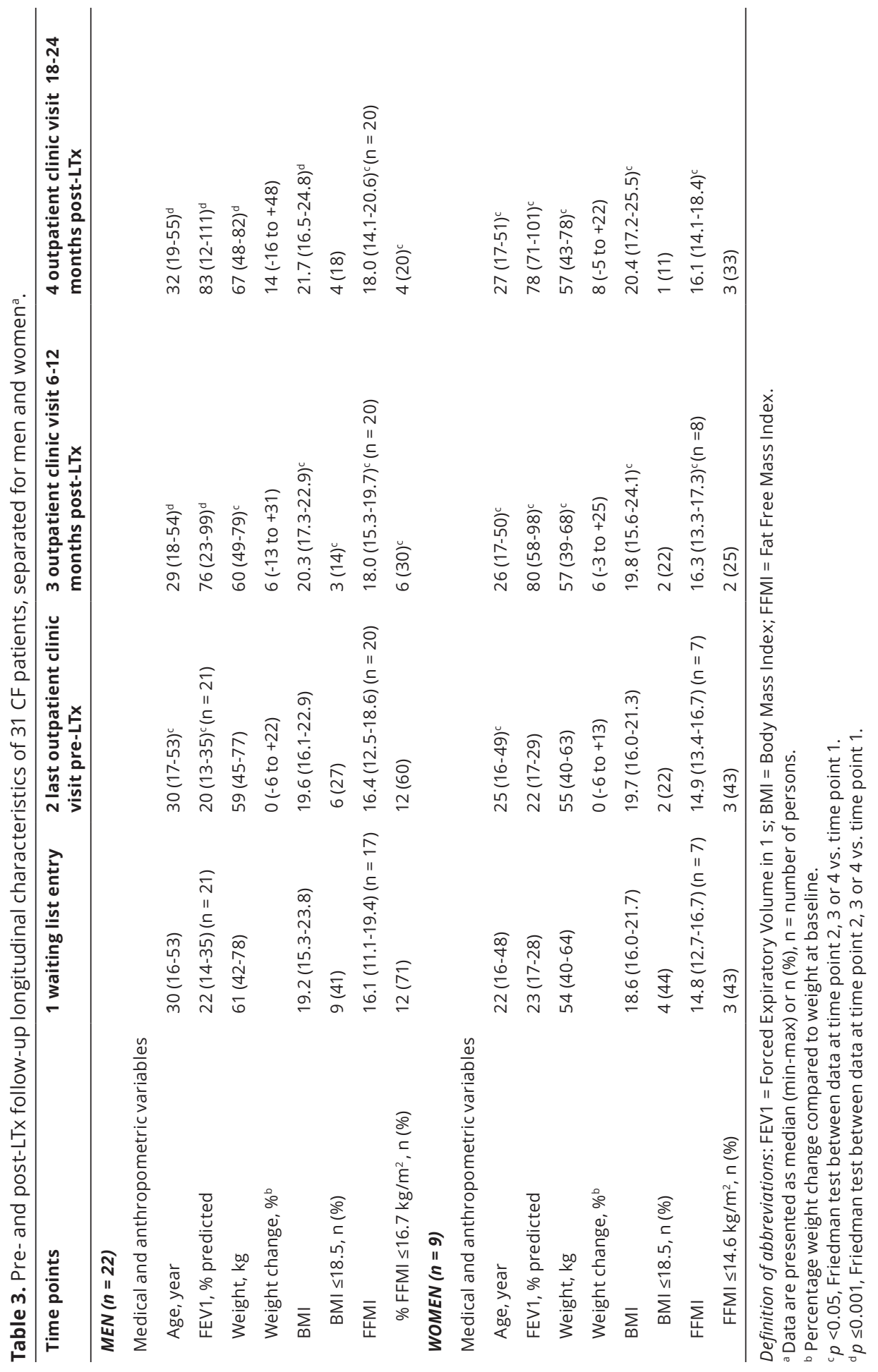




\section{DISCUSSION}

This study was performed to describe changes in nutritional status in pre-and post-LTx CF patients, and how this relates to dietetic interventions and survival. At the entrance of the LTX programme, most patients had a long history of dietetic care and were familiar with nutritional support. However, almost $50 \%$ of the patients did not meet the recommended intake level of energy at baseline and $36 \%$ of the patients were underweight based on their BMI. Nutritional support during the pre-LTx period was unable to improve body weight or FFM, although further deterioration was prevented. After LTX, bodyweight, BMI and FFMI improved gradually.

Our study was limited by its retrospective approach; not all data could be retrieved. For 12 LTX patients who died within one year after transplantation no medical records were available. It would have been interesting to know their baseline BMI and FFMI. We assume that the use of electronic patient files will facilitate follow-up studies in the future. Secondly, we only used data from the outpatient clinic. Sometimes patients are hospitalized for more than 6 months and this may have an impact on bodyweight and composition before LTx.

Compared to other studies in pre- and post-LTx patients, our study population had lower values for body weight, BMI and FFMI.1723,27,28 This may be explained not only by treatment differences, but also by differences in study population (other LTX-studies included populations with chronic obstructive pulmonary disease, emphysema and bronchiectasis) or differences in cut off points used to classify underweight (e.g. BMI varying from $<17$ to $<21 \mathrm{~kg} / \mathrm{m}^{2}$ ). In our data a weak association was suggested between low baseline FFMI and survival, as was seen in earlier studies. ${ }^{2,3}$ Although this appears to be plausible, follow up data of larger study populations are needed to confirm this.

European and Dutch nutritional guidelines recommend an intake of $120-150 \%$ of the estimated average requirement for energy for CF individuals compared to healthy peers. ${ }^{19}$ In our preLTx population $48 \%$ failed to achieve recommended energy intake and $20 \%$ failed to achieve recommended protein intake. This percentage is lower than described by White et al. where $72 \%$ of the general CF population failed to achieve recommended energy- and protein intake. ${ }^{31}$ However, this may be due to differences in absorption factor, Activity Factor and/or disease factor that are used to calculate total energy expenditure. We used an absorption factor of 0.93 for pancreatic insufficient patients but factors between 0.85 and 0.9 and thus higher energy requirements have been proposed. ${ }^{32}$ Moreover, the disease factor was now set at 1.3 for all patients but this may be too low during periods of respiratory tract infection. It 
is therefore possible that we underestimated energy requirements, which might explain why improvement of BMI and FFMI was not seen pre-LTx. To our knowledge, only one study described the dietary intake of pre- and post-LTx patients with CF, suggesting that despite a high caloric intake these patients remain malnourished before LTX while post-LTx they became well-nourished despite a drop in caloric intake. ${ }^{29}$ Future research should explore actual energy demands in pre-and post-LTx patients with CF using measured instead of calculated energy expenditure data.

During the waiting list period we advised ONS and/or tube feeding in patients who were unable to maintain or improve body weight. Kalnins et al. compared the effects of oral dietary supplements with dietary counselling on energy intake and nutritional status in malnourished CF patients, and reported that over a 3-month period neither sip feeding alone nor dietary counselling improved nutritional status. ${ }^{33}$ In our study half of the patients used ONS at baseline. ONS is meant to be an addition but may replace dietary intake when appetite is worsened due to a disease. In that case advising ONS may have the opposite of the desired effect. PEG tube feeding may not have this effect. In fact, it was shown to improve nutritional status in patients with CF and with end-stage lung disease in two studies. ${ }^{34,35}$ Possibly, more aggressive nutritional therapy with PEG tube feeding may be necessary to stimulate nutritional rehabilitation before LTX. Whether this improves survival needs to be evaluated.

After transplantation significant improvements in weight, BMI and FFMI were found for both men and women. A limited number of studies consistently found that body weight improved after LTx. ${ }^{28,30}$ Singer et al. found that weight gain was greatest in patients with CF and bronchiectasis compared to other LTx groups. ${ }^{28}$ The weight gain in our study could have been overestimated because some of the patients had their LTx before the age of 18. At waiting list entry eight patients were between 15 and 18 years old. Age related increases in weight and BMI could appear as successful nutritional interventions and therefore overestimate the effect of dietetic intervention. However, we expect that this effect only marginally influenced our analyses, because only 4 of these young patients were included in the nutritional followup analyses. In accordance with our findings, Habedank found that weight gain was highest in underweight patients. ${ }^{30}$ In our study, this was more pronounced in men than in women. Possibly women feel more comfortable with their lower body weight. This phenomenon is also described by Abbott et al. who found that women with CF reported more dieting behaviour, greater preoccupation with food and more pressure from others to eat than CF males. ${ }^{36}$ 
Although on average body weight increased after LTx, we observed a wide variation in weight change between patients. Postoperative weight loss may be explained by specific complications during the clinical course after LTx. One patient required re-transplantation, another one had a severe infection, and in one patient the PEG tube was not accessible. In other words, several causes may lead to unwanted weight loss. Weight gain post-LTx may have been affected by corticosteroid therapy, which was administered to all patients after LTX. However, we expect this effect to be minimal, because after 7-12 months daily prednisone dose was minimized to $10 \mathrm{mg}$ in the majority of patients. Various studies have shown that the relation between corticosteroid dose and weight gain in transplant populations is not significant. ${ }^{27,37,38}$

In conclusion, a low BMI and especially a low FFMI at waiting list entry appears to impair survival in CF patients who are LTX candidates. Patients maintained a low body weight before LTX despite the use of additional ONS and/or tube feeding. After LTx weight gain is achieved, and more pronounced in patients who start with underweight. More research is needed on the impact of nutritional status on prognosis, on the specific nutritional requirements at several stages of the treatment process and on the effectiveness of several nutritional support measures. 


\section{REFFERENCE LIST}

1. Spahr JE, Love RB, Francois M, Radford K, Meyer KC. Lung transplantation for cystic fibrosis: current concepts and one center's experience. J Cystic Fibros 2007;6(5):334-50.

2. Madill J, Gutierrez C, Grossman J, et al. Nutritional assessment of the lung transplant patient: body mass index as a predictor of 90-day mortality following transplantation.J Heart Lung Transplant 2001;20(3):288-96.

3. Schwebel C, Pin I, Barnoud D, et al. Prevalence and consequences of nutritional depletion in lung transplant candidates. Eur Respir J 2000;16(6):1050-5.

4. Bell SC, Bowerman AR, Davies CA, Campbell IA, Shale DJ, Elborn JS. Nutrition in adults with cystic fibrosis. Clin Nutr 1998;17(5):211-5.

5. Dray X, Kanaan R, Bienvenu T, et al. Malnutrition in adults with cystic fibrosis. Eur J Clin Nutr 2005;59(1):152-4.

6. Hollander FM, De Roos NM, De Vries JHM, Teding van Berkhout F. Assessment of nutritional status in adult patients with cystic fibrosis: whole-body bioimpedance vs body mass index, skinfolds, and leg-to-leg bioimpedance. J Am Diet Assoc 2005;105:549-55.

7. Lederer DJ, Wilt JS, D'Ovidio F, et al. Obesity and underweight are associated with an increased risk of death after lung transplantation. Am J Respir Crit Care Med 2009;180(9):887-95.

8. Kastner-Cole D, Palmer CN, Ogston SA, Mehta A, Mukhopadhyay S. Overweight and obesity in deltaF508 homozygous cystic fibrosis. J Pediatr 2005;147(3):402-4.

9. Stephenson AL, Mannik LA, Walsh S, et al. Longitudinal trends in nutritional status and the relation between lung function and BMI in cystic fibrosis: a population-based cohort study. Am J Clin Nutr 2013;97(4):872-7.

10. Allen JG, Arnaoutakis GJ, Weiss ES, Merlo CA, Conte JV, Shah AS. The impact of recipient body mass index on survival after lung transplantation. J Heart Lung Transplant 2010;29(9): 1026-33.

11. Ionescu AA, Nixon LS, Evans WD, et al. Bone density, body composition, and inflammatory status in cystic fibrosis. Am J Respir Crit Care Med 2000;162(3 Pt 1):789-94.

12. King SJ, Nyulasi IB, Strauss BJ, Kotsimbos T, Bailey M, Wilson JW. Fat-free mass depletion in cystic fibrosis: associated with lung disease severity but poorly detected by body mass index. Nutrition Jul-Aug 2010;26(7-8):753-9.

13. Esteves GB, Macedo A, Nakasato M, Bruno ML. Evolution of nutritional status in lung transplant candidates who are initially malnourished or overweight. J Bras Pneumol 2009;35(12):1217-9.

14. Forli L, Pedersen JI, Bjortuft O, Vatn M, Boe J. Dietary support to underweight patients with endstage pulmonary disease assessed for lung transplantation. Respiration 2001;68(1):51-7.

15. Sims EJ, Green MW and Mehta A. Decreased lung function in female but not male subjects with established cystic fibrosis-related diabetes. Diabetes Care 2005;28(7):1581-7.

16. American Diabetes Association. Diagnosis and classification of diabetes mellitus. Diabetes Care 2011; 34(Suppl. 1):S62-9.

17. Eurotransplant Internation Foundation. http://www.eurotransplant.org/cms/ [Exist:01-09-2012].

18. WHO. Physical status: the use and interpretation of anthropometry. Report of a WHO Expert Committee, 854., World Health Organ Tech Rep Ser;1995. p.1-452. 
19. Sinaasappel M, Stern M, Littlewood J, et al. Nutrition in patients with cystic fibrosis: a European Consensus. J Cystic Fibros 2002;1(2):51-75.

20. TNO Growth Charts. Accessible through. www.tno.nl [available in english].

21. Jaffrin MY, Morel H. Body fluid volumes measurements by impedance: a review of bioimpedance spectroscopy (BIS) and bioimpedance analysis (BIA) methods. Med Eng Phys 2008;30(10):1257-69, http://dx.doi.org/10.1016/j.medengphy.2008.06.009 [Epub 2008 Aug 3].

22. Spicher V, Roulet M, Schaffner C, Schutz Y. Bio-electrical impedance analysis for estimation of fat-free mass and muscle mass in cystic fibrosis patients. Eur J Pediatr 1993;152(3):222-5.

23. Kyle UG, Schutz Y, Dupertuis YM, Pichard C. Body composition interpretation. Contributions of the fat-free mass index and the body fat mass index. Nutrition 2003;19(7-8):597-604.

24. Schofield WN. Predicting basal metabolic rate, new standards and review of previous work. Hum Nutr Clin Nutr 1985;39(Suppl. 1):5-41.

25. Taminiau JAJM, de Meer K, Hofman Z. Bepaling van voedingsbehoeften. In: Taminiau JAJM, de Meer K, Kneepkens CMF, Verheul-Koot MA, Lafeber HN, editors. Werkboek enterale voeding bij kinderen. Amsterdam:VU Boekhandel/Uitgeverij BV; 1997. p. 26-47.

26. Griffiths RD, Bongers T. Nutrition support for patients in the intensive care unit. Postgrad Med J 2005;81:629-36.

27. Kyle UG, Nicod L, Romand JA, Slosman DO, Spiliopoulos A, Pichard C. Four-year follow-up of body compostion in lung transplant patients. Transplantation 2003;75(6):821-8.

28. Singer LG, Brazelton TR, Doyle RL, Morris RE, Theodore J. Weight gain after lung transplantation. J Heart Lung Transplant 2003;22(8):894-902.

29. Madill J, Maurer JR, de Hoyos A. A comparison of preoperative and postoperative nutritional states of lung transplant recipients. Transplantation 1993;56(2):347-50.

30. Habedank D, Ewert R, Hetzer R, Anker SD. Reversibility of cachexia after bilateral lung transplantation. Int J Cardio/ 2009;133(1):46-50.

31. White H, Morton AM, Peckham DG, Conway SP. Dietary intakes in adult patients with cystic fibrosis-do they achieve guidelines? J Cystic Fibros 2004;3(4):1-7.

32. Wouthuyzen-Bakker M, Bodewes FA, Verkade HJ. Persistent fat malabsorption in cystic fibrosis; lessons from patients and mice. J Cystic Fibros May 2011;10(3):150-8.

33. Kalnins D, Corey M, Ellis L, Pencharz PB, Tullis E, Durie PR. Failure of conventional strategies to improve nutritional status in malnourished adolescents and adults with cystic fibrosis. J Pediatr 2005;147(3):399-401.

34. Wengrower D, Goldin E, Kerem E, Kramer MR. Role of percutaneous endoscopic gastrostomy in a malnourished patient awaiting lung transplant. Transplant Proc 1997;29(6):2655-6.

35. Williams SG, Ashworth F, McAlweenie A, Poole S, Hodson ME, Westaby D. Percutaneous endoscopic gastrostomy feeding in patients with cystic fibrosis. Gut 1999;44(1):87-90.

36. Abbott J, Morton AM, Musson $\mathrm{H}$, et al. Nutritional status, perceived body image and eating behaviours in adults with cystic fibrosis. Clin Nutr 2007;26(1):91-9.

37. van den Ham EC, Kooman JP, Christiaans MH, Nieman FH, van Hooff JP. Weight changes after renal transplantation: a comparison between patients on 5-mg maintenance steroid therapy and those on steroid-free immunosuppressive therapy. Transpl Int 2003;16(5):300-6.

38. Williams JJ, Lund LH, LaManca J, et al. Excessive weight gain in cardiac transplant recipients. J Heart Lung Transplant 2006;25(1):36-41. 
蛋

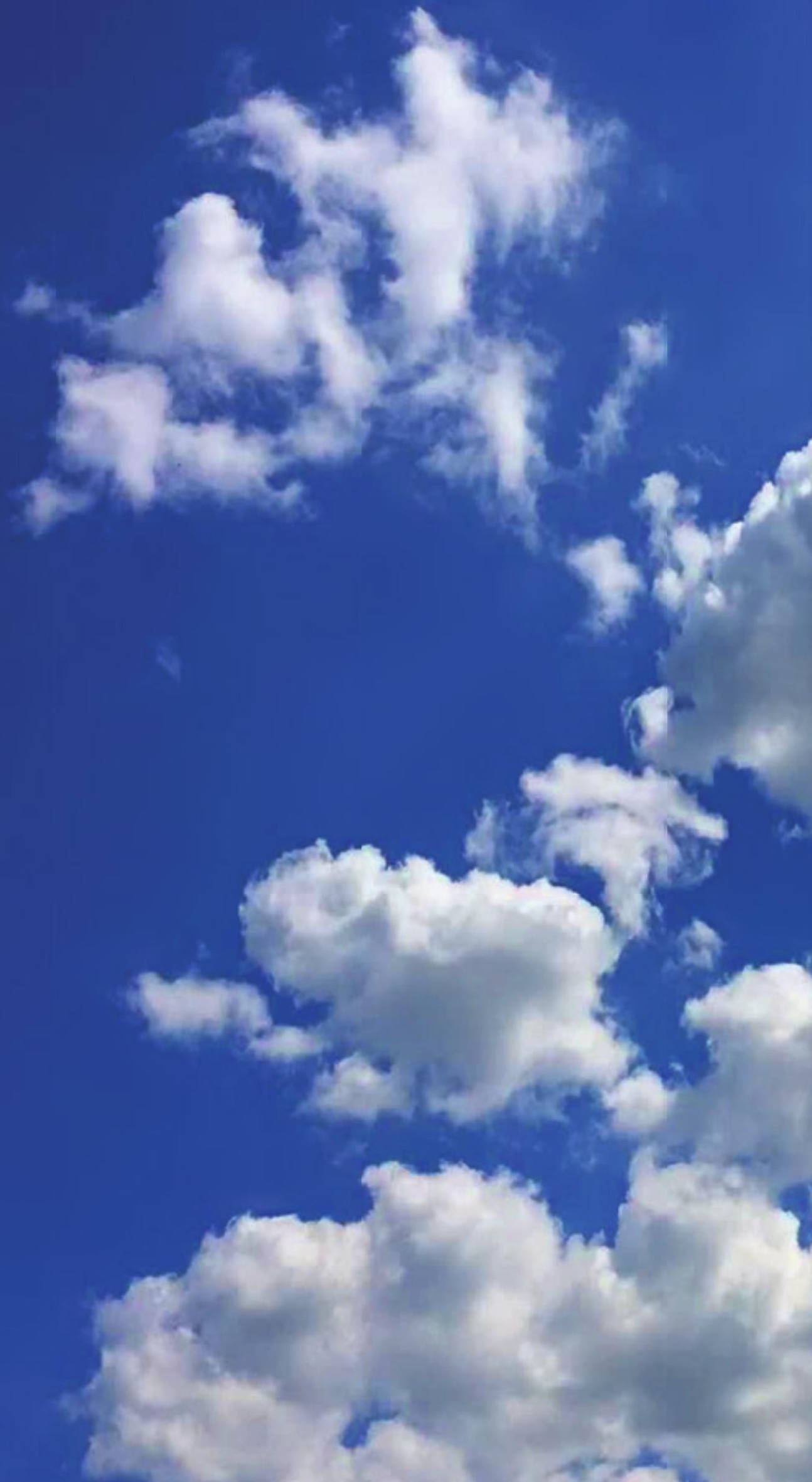




\section{Chapter 8}

\section{General Discussion and Summary}




\section{CLINICAL EVIDENCE AND FUTURE PROSPECTS IN NUTRITIONAL THERAPY IN CYSTIC FIBROSIS}

'Let food be thy medicine and medicine be thy food' is a well-known quote by Hippocrates.

Although this Greek scientist died 370 years BC, his statement is still adopted by dietitians and physicians worldwide. In contemporary life, the benefits of healthy nutrition are important for all individuals, with or without a chronic disease. For patients with $\mathrm{CF}$, nutrition is also an important issue since it affects pulmonary function and survival. ${ }^{1-3}$ This importance is reflected in European and Australian guidelines that include nutritional therapy and the monitoring of nutritional status as part of disease management in CF.4,5

Nowadays, dietetics is largely practice-based. To further improve the quality of patient care, it is of great importance to enhance the scientific credibility of dietetics based on evidence-based nutritional recommendations, methods and techniques. However, randomized controlled trials (RCT's) are not easy or even unethical to perform in clinically vulnerable individuals, and therefore most of the nutritional interventions are described in observational studies. The main problem in observational studies is the presence of confounders and selection bias, ${ }^{6}$ which means that conclusions on cause and effect cannot be inferred from these studies. In recent years, data from observational studies have become an increasingly important source of evidence because of improvements in observational-study methods and advances in statistical analysis. ${ }^{7}$ Well-designed observational studies can play a key role in supporting the evidence base for nutritional therapies and may be the best way to answer the many nutritional questions in situations in which the classical RCT approach does not apply.

In this thesis we investigate the nutritional challenges in adults with CF before and after lung transplantation (LTX). Anthropometric measurements provide important information for the evaluation of the nutritional status in our CF study population. Furthermore, we explore the effectiveness of nutritional interventions in adults with end-stage CF both before and after LTx. The studies in this thesis address the lack of knowledge about nutritional assessment and dietary interventions in this group of individuals. Our studies are based on longitudinal cohorts, especially in adults with CF with end-stage lung disease. The presence of this specific group of individuals in our centre makes it possible to conduct unique research. However, the outcomes of studies of specific groups can limit the extent to which the results can be generalized to other individuals with CF. The results may be biased as these individuals already have a worse nutritional status compared to the general population with CF. Besides, cross- 
sectional research can be used to compare methods and find associations, but not to study cause-effect relationships. A recent Cochrane database of systematic reviews on enteral tube feeding (ETF) states that performing an RCT would be difficult due to the ethics of withholding an intervention with ETF in a group of people whose nutritional status necessitates it. ${ }^{8}$ ETF is advised in CF individuals who are unable to consume adequate amounts of calories and protein to meet growth and weight maintenance goals. ${ }^{9}$ Furthermore, ETF is effective especially in malnourished CF individuals. ${ }^{10}$ However, the existing data is inadequate to provide recommendations concerning the use of ETF as a method to improve or to stabilize pulmonary function. ${ }^{9}$ Even though there are no RCT's to decisions regarding the use of ETF in CF patients, multiple retrospective studies suggest that its use can improve age-dependent anthropometrics. ${ }^{9}$ These age-dependent anthropometrics include weight/age percentile, height/age percentile and weight-for-height percentile for $0-2$ years; stature/age percentile and body mass index (BMI) percentile for 2-20 years; and BMI value for adults. ${ }^{4}$

The outcomes of nutritional interventions in clinical practice are of great value for both the individual patient, and the medical staff. In fact, patients' adherence improves if they receive clear information about effective treatment options and dieticians identify the wide variety of nutritional concerns in CF patients adequately. ${ }^{11}$ Moreover, the results of dietetic interventions contribute to practice-based evidence and result in modifications to current nutritional therapy. Our studies are performed in end-stage CF individuals and are generalizable to other CF lung transplant centres. However, in low-socioeconomic countries, CF treatment options are limited with the risk of poorer health outcomes..$^{12} \mathrm{LTX}$ will not be possible in every CF centre and the use of measurement tools may also be limited by financial constraints. The collaboration with CF expertise centres should be intensified to share knowledge and equipment to reduce the discrepancies in health outcomes of CF individuals.

In addition, the availability of cystic fibrosis transmembrane conductance regulator (CFTR) modulator therapy is not common worldwide..$^{13}$ As a result of this dissimilarity, the clinical outcomes and nutritional status of patients can diverge in the CF population worldwide. The benefits of CFTR modulators are associated with the improvement of appetite and protein anabolism, combined with reduction of work of breathing, inflammation and energy expenditure. ${ }^{14}$ Furthermore, improvements in fecal elastase- 1 levels are observed in children with $\mathrm{CF}^{15}$ resulting in an increased absorption of fat. Consequently, the effects of CFTR modulators will change dietary recommendations for energy ${ }^{14}$ as improvements in weight and $\mathrm{BMI}$ are observed. ${ }^{16}$ The change in CF energy requirements needs to be explained to CF individuals, their families and caregivers. Moreover, additional focus on the nutritional values 
of protein is warranted as currently, evidence-based recommendations for daily protein intake are lacking and further studies are needed to develop guidelines for protein thresholds.,17 Specific nutritional treatment goals for each individual with CF are warranted. ${ }^{18}$ The importance of personalized nutritional treatment is increasing as some CF individuals develop normal weight obesity ${ }^{19}$ related to this CFTR modulator therapy, whereas others still have difficulties achieving and improving their nutritional status. Monitoring body composition and nutrient intake at the annual outpatient visit provides information of the nutritional problems the current CF individual can experience. Diagnostic tools as measurement of body composition by bioelectrical impedance, and energy needs by indirect calorimetry, will be most valuable in fine-tuning and monitoring dietetic interventions in the near future.

In the following paragraphs, we describe the results from our studies. At the end of each chapter, we discuss future prospects consecutively. And finally, we indicate directions for new research as CFTR modulator therapy is becoming more widely available in CF treatment, the life expectancy of CF patients is increasing, and survival in CF LTx patients is improving.

Chapter 2 describes the Latest evidence and recommendations for an optimal approach to nutrition in CF patients. In this review, we explored the most recent findings regarding nutritional management associated with pulmonary function and outcome. Evidenceinformed and practice-based guidelines on nutritional care of infants, children and adults with CF have been developed by the ESPEN-ESPGHAN-ECFS. ${ }^{4}$ Since 2016, excellent guidelines and systematic reviews have been published to endorse the importance of adequate nutritional treatment at different age and disease stages in $\mathrm{CF}^{5,9,20}$ The standard nutritional care for CF is a high calorie, high-fat diet with pancreatic enzyme replacement therapy (PERT) and fatsoluble vitamin supplementation to achieve an adequate nutritional status. ${ }^{4}$ CFTR modulators, including correctors and potentiators, are now available in clinical practice with positive effects on nutritional status. ${ }^{14,21,22}$ In combination with a knowledge of the genetic modifiers of the disease, the individual prognosis will be improved using individualized therapy. The optimal treatment for each CF patient can be achieved by combining personalized medical treatment, adequate nutritional intervention and improved life style behavior. Nowadays, dietary intervention in CF care needs adaptation as CFTR modulation affects the nutritional status and pulmonary function of individuals. The increased appetite of CF patients is one of the beneficial side effects of CFTR modulators. This beneficial side effect may contribute to unwanted weight gain and the unfavorable increase of fat mass. An unusually high percentage of fat mass with a normal weight, defined as normal weight obesity, ${ }^{17}$ and obesity in CF patients occurs more frequently. ${ }^{9}$ Therefore, the implementation of changes in nutritional 
recommendations is needed. In patients using CFTR modulators, the measurement of REE needs to be expanded to investigate changes in energy metabolism. There is some evidence that a decrease in REE occurs. ${ }^{14,23}$ To prevent weight gain by an increase of fat mass in CF individuals, current recommendations for energy needs to be reconsidered. A new focus on a balanced diet and a healthy active lifestyle is necessary to achieve a good body composition with normal fat and fat free mass proportions. In fact, nutritional care should be closely adapted to the various stages and complications facing CF individuals across the lifespan to extend survival and to improve their quality of life. For this individualized nutritional treatment of CF patients, the knowledge, and the clinical experience, of a specialized CF dietitian is warranted.

In a contemporary lifestyle, e-health can be used in dietetic CF care to inform patients adequately. A specialized CF dietitian could use e-health consultations to answer specific questions related to malnutrition, sports, cystic fibrosis related diabetes, and weight or obesity problems. ${ }^{11,24}$ Blended care, where digital health and usual care are integrated, could contribute to supporting and motivating patients to adopt healthier eating habits which could prevent the development of unwanted comorbidities. This e-mental health service is a new era of medicine. Patients are empowered by increased access to information and their providers. ${ }^{25,26}$ In the near future, health care apps should demonstrate their effectiveness in meeting clinical and engagement end points in the long term. Current available CF apps are mainly used to record symptoms and act as reminders for medications and meals. ${ }^{27}$ In further research, apps could be used to collect data from larger groups of CF individuals, minimising the necessity of them having to come to the hospital.

\section{Chapter 3 shows that Resting energy expenditure (REE) is underestimated by prediction} equations in patients with end-stage CF. In general, to determine total energy requirements in patients, prediction equations are used in which resting energy expenditure (REE) is multiplied by activity and disease factors. ${ }^{28,29} \mathrm{REE}$ is an important determinant of total energy expenditure (TEE). In healthy persons, REE can be calculated based on age, sex, and body weight using different prediction equations such as those of Harris-Benedict (HB; 1919 and 1984), Schofield, and the World Health Organization (WHO;1985). ${ }^{28-30}$ For CF patients, there is no consensus on which prediction equation is most reliable. When using prediction equations, overestimation or underestimation of REE occurs. ${ }^{31}$ This may lead to inadequate individual recommendations for TEE, and impairment of the nutritional status. Measured REE values will improve the estimation of TEE, and provide adequate energy recommendations. Indirect calorimetry (IC) is the most commonly used and is a non-invasive method for measuring 
REE in clinical populations including CF. ${ }^{32,33}$ Our study is the first study that assessed the agreement between measured REE with IC and predicted REE according to Harris-Benedict 1919 and 1984, Schofield, and WHO 1985 prediction equations. Moreover, this study is the first that assessed differences in REE adjusted for fat free mass in pre- and post-LTX CF patients. REE estimated by prediction equations was adequate in a maximum of $25 \%$ of pre-LTX CF patients and $67 \%$ of post-LTX CF patients. Furthermore, in our study population, REE was underestimated using prediction equations in all pre-LTx patients with CFRD, colonized with $P$ aeruginosa and with a homozygote $\Delta$ F508 mutation. Within 2 years post-LTX, in 4 out of 12 post-LTX CF patients REE was still underestimated using prediction equations, possibly due to re-colonisation with $P$ aeruginosa from the patient's paranasal sinuses. When REE was adjusted for body weight or fat free mass (FFM) a significant difference was seen between pre- and post-LTX CF patients: REE/kg and REE/kg FFM was significantly higher in pre- than in post-LTX CF patients. These differences may be explained by a decrease of inflammation and pulmonary infections post-LTx and a decline of respiratory effort. This study was limited by its cross-sectional design. However, the two patient groups were similar in characteristics (except for pulmonary function) and therefore suitable to draw conclusions from.

IC is also the reference standard for measurement of energy expenditure in critically ill patients. ${ }^{34}$ For further research purposes, IC should be conducted at the intensive care unit (ICU). Moreover, technological advancement makes it possible to perform measurements of energy expenditure in clinic patients at the bedside using valid and accurate portable devices. ${ }^{35,36}$ In future research, clinically ill CF patients waiting for LTX at the ICU can be included. ICU patients often suffer from chronic critical illness causing an increase in energy expenditure, leading to proteolysis and related muscle loss. Discrepancies between the estimated and measured energy expenditure influence nutritional management and can have a negative impact on the outcomes of critically ill CF patients. Optimal supplementation and modulation of caloric and protein intake can avoid under- or overfeeding. ${ }^{37}$ Under- or overfeeding are both associated with poorer outcomes. ${ }^{38}$ IC is the preferred method to estimate resting energy expenditure and provides support for the appropriate caloric intake in critically ill patients to counter energy and muscle loss. ${ }^{39}$ This method can be used additionally in dietetic practice, especially in patients with unwanted weight loss or weight gain.

\section{The implications for clinical practice and research of non-fasting bioelectrical} impedance analysis in CF are investigated in Chapter 4. Body composition may affect pulmonary function in CF patients and can be monitored by using bioelectrical impedance analysis (BIA)..$^{19,40}$ In this study, we assessed whether the differences between fasting and 
non-fasting BIA measurements are clinically relevant in adult CF patients. In order to make the results applicable in clinical research, this study additionally investigated whether associations between BIA results with pulmonary function are different when using fasting or non-fasting data. All adult CF patients underwent whole body single frequency BIA measurements at the outpatient clinic, before and after their nutritional and fluid intake in the morning. BIA measures the resistance of the body to a small electrical current, and can indicate body composition. Nutritional and fluid intakes significantly increase FFM estimates by $0.23 \mathrm{~kg}$, and significantly lower FM estimates by $0.22 \mathrm{~kg}$, which is not clinically relevant. Furthermore, in $86 \%$ of the patients the difference in FFM and FM estimates after nutritional and fluid intake was less than $1 \mathrm{~kg}$. After adjusting FFM and FM for height, the estimates remained similar in $83 \%$ of the patients. These results show that classification of patients is similar before and after nutritional and fluid intake. The associations between BIA results and pulmonary function remained similar before and after nutritional and fluid intake. These results also indicate that non-fasting BIA measurements can be registered in electronic patient records and that researchers may use these data for research purposes. However, the study results should be interpreted cautiously when applied to other body composition devices. Reproducibility increases when measurements over time are performed using similar testing conditions. Moreover, the single frequency BIA provides raw data (impedance, resistance, reactance, and phase angle). These raw data were registered in electronic medical records. Estimates of FFM (in $\mathrm{kg}$ ), \%, and $\mathrm{kg} / \mathrm{m}^{2}$ ), and FM (in $\mathrm{kg}, \%$, and $\mathrm{kg} / \mathrm{m}^{2}$ ) were obtained, using the Kyle equation. ${ }^{41}$ The Kyle equation was used to estimate body composition, and is commonly used in dietetic practice as it shows good precision when validated against Dual-energy X-ray absorptiometry (DXA) among healthy individuals, as compared to other BIA equations..$^{42}$ Our data were also analysed with the Charatsi formula, which has been validated in CF children, ${ }^{43}$ but our conclusions remained similar when comparing the data with the results from the Kyle equation.

Therefore, BIA measurements are implemented in our CF centre and performed in adult CF patients in non-fasting state visiting the outpatient clinic. Patients experience no discomfort and are very interested in their measurement outcomes. The nutritional status of CF individuals can be monitored by using follow up data in order to optimize pulmonary function. Furthermore, the effect of nutritional and physical interventions can be evaluated more adequately. Using follow up data, these interventions can be adjusted if necessary. This is of great importance to increase treatment adherence of patients. So, BIA can easily be used as a prognostic and body composition assessment tool. ${ }^{44}$ However, for clinically unstable CF patients, additional techniques are needed to evaluate their nutritional status as oedema 
and dehydration can lead to less reliable results using BIA..$^{45}$ Other techniques, for example the Dual-energy X-ray absorptiometry (DXA), can be used to measure body composition. This is a more expensive method, which is also of great value in studying sarcopenia ${ }^{46}$ and bone health. As the life expectancy of CF patients is increasing, it is important to monitor bone health in both children and adults. Therefore, future research could focus on the validation of BIA against DXA for body composition measurements in CF adults.

\section{Resting energy expenditure in CF patients decreases after LTX, which improves applicability of prediction equations for energy requirement, is reported in Chapter $\mathbf{5}$.}

The decision to place a patient on the waiting list for LTX is complex, reflecting considerations not only of clinical and psychosocial characteristics of the individual patient but also programspecific factors and the influence of a lung allocation system (LAS). The selection of appropriate LTX candidates is multifaceted and is a result of a process that carefully considers the unique aspects of each patient and each transplant program. Because LTx is a complex therapy with a significant risk of perioperative morbidity and mortality, it is important to consider the overall sum of contraindications and comorbidities. In a consensus document that provides a list with absolute and relative contraindications, progressive or severe malnutrition is listed as a relative contraindication for bilateral $\mathrm{LTX} .{ }^{47}$ Therefore, specialized dietary counselling is warranted for CF patients to optimize their nutritional status before surgery. ${ }^{48}$ Resting energy expenditure (REE) is used to estimate total energy expenditure and to provide an adequate personalized dietary advice. In previous clinical studies and current practice, REE is commonly estimated by using prediction equations and is underestimated in CF patients especially before LTX. ${ }^{33,49-51}$ However, this has not been studied after LTX. In our unique longitudinal study, we investigated the change in REE in CF patients before and after LTX with a follow-up of 2 years. We also compared the results of REE with those of prediction equations. ${ }^{28-30,52}$ Our study confirmed the findings of our cross-sectional study: REE and REE adjusted for kilogram fat free mass (FFM) significantly decrease after LTx. Moreover, prediction equations are inaccurate until one year after LTX. From then on, REE and REE/kg FFM remain stable and prediction of REE using equations is accurate (i.e. within $10 \%$ of measured value) in about two thirds of the patients. To prevent underestimations of energy needs, we recommend using the measured REE by IC instead of using prediction equations for REE in patients with CF and end-stage lung disease until one year after LTX.

Future studies are encouraged to validate our observations and perform subgroup analysis with more patients included. It is of great interest to investigate whether the REE will change more than 2 years after LTX in a larger sample of patients with CFRD or new onset diabetes 
after transplant (NODAT). The incidence of these complications is high. ${ }^{53,54}$ Moreover, as the physical condition of the patient after LTx may improve, an increase in exercise may also alter the total energy demands. The measurement of REE, in combination with monitoring body composition and the physical activities of patients with transplants may contribute to optimally individualized treatments leading to better survival outcomes. It would also be interesting to use the double-labelled water method to determine total energy costs, which has not yet been investigated in adult CF patients but is already a valid method in healthy persons. ${ }^{55}$ In addition, the use of immunosuppressive drugs like corticosteroids is related to comorbidities ${ }^{56}$ and may result in a change of eating habits. Therefore, a specialized CF LTX dietitian is warranted to advise patients regarding adequate energy requirements in post-transplant CF patients to prevent the development of unwanted comorbidities such as metabolic syndrome ${ }^{57}$ and dyslipidemia.

In Chapter 6, we show that Body weight and body mass index in patients with end-stage cystic fibrosis stabilize after the start of enteral tube feeding. Enteral nutrition support is widely used to improve nutritional status in CF patients. ${ }^{10}$ This type of support includes oral nutrition supplementation and enteral tube feeding (ETF). ETF is considered when oral nutrition supplementation fails to improve BMI or to prevent weight loss. ${ }^{9}$ Depending on the expected duration of nutritional support, the physical and gastrointestinal tract condition, and/or patient preference and choice, ETF can be administered by nasogastric, nasoduodenum, percutaneous endoscopic gastrostomy (PEG) or percutaneous endoscopic jejunostomy tube. ${ }^{58}$ The latter is an alternative method for patients with end-stage lung disease. ${ }^{59}$ Previous studies have been limited to CF patients with moderately or mildly impaired pulmonary function and improvements in nutritional status were associated with a higher percentage of CF patients surviving for 2 years or more. ${ }^{60,61}$ The effectiveness of ETF in improving the nutritional status and survival in CF patients with end-stage lung disease has not been investigated. A difference in survival between sexes is also described; women with CF have a shorter life expectancy than men with CF. ${ }^{62,63}$ No studies have investigated the differences between adult men and women with CF in clinical outcomes after ETF was started. Therefore, we performed a retrospective study with the aim to determine the effects of ETF in men and women with CF and end-stage lung disease on body weight, BMI, and pulmonary function. Data on nutritional and clinical outcomes of 26 patients with end-stage $C F$, including weight, height, BMI, and pulmonary function (FEV1\% predicted) were collected during usual outpatient clinic visits at different points in time from 6 months before to 6 months after starting ETF. Six months after starting ETF, we found a mean increase in body weight of 3.5 $\mathrm{kg}$ and a mean increase of $1.3 \mathrm{~kg} / \mathrm{m}^{2}$ in BMI for the entire group of patients. FEV1\% predicted 
was and remained low; $28 \% 6$ months before the start of ETF, $26 \%$ at the start of ETF and $25 \% 6$ months after the start of ETF. The conclusion of our study is that ETF improved body weight and BMI but not pulmonary function in our patients with end-stage CF. The clinical outcomes were similar in women and men, but the sample size for men alone was too small to reach statistical significance.

Reported use of ETF suggests that it results in nutritional and respiratory improvement. However, efficacy has not been assessed by randomized controlled trials (RCT's). To perform a RCT is difficult due to the ethics of withholding an intervention in a group of patients whose nutritional status necessitates it. ${ }^{64}$ Larger prospective observational studies, providing more opportunity to correct for confounders, are needed to further describe the effects of ETF. Furthermore, hyperglycemia ${ }^{65}$ and gastro-intestinal discomfort ${ }^{66}$ can occur in CF patients or CF-related diabetes using nocturnal ETF. Therefore, further research could focus on adequate supplementation of pancreatic enzyme replacement therapy (PERT) in CF patients using ETF. A novel in-line digestive cartridge with immobilized lipase (RELiZORB) has been designed to address the unmet need for PERT in patients using ETF. ${ }^{67}$ Unfortunately, this cartridge is not available in European countries yet. This, or a similar, product will be an interesting tool to use for the improvement of ETF efficacy in pancreatic insufficient CF patients. More research is warranted to reduce these complaints in CF patients and to improve clinical outcomes.

The effects of nutritional status and dietetic interventions are important for survival in CF patients before and after LTx. The results of this study are described in Chapter 7. Pre- and post-transplant nutritional management aims to maintain or improve nutritional status, in order to meet the criteria for LTx and optimize survival before and after LTX. LTX is an established treatment option for end-stage lung disease, also in CF patients. However, malnutrition is a common problem in CF patients. ${ }^{68,69}$ Only a few studies have investigated nutritional intervention strategies in patients on the waiting list for LTX. ${ }^{70-72}$ The aim of our study was to describe nutritional status and intake at waiting list entry, subsequent changes in body weight and fat free mass (FFM) before and after LTX, and survival after LTx using data from out-patient visits. We could retrieve data from 75 CF patients from our own patient files. Fifty one of the 75 patients received a transplant. Two-year complete follow-up data on anthropometry and diets were available for 31 of these patients: 10 died after LTx and 10 patients were followed for less than 1 year. On entering the LTx programme, most patients had had a long history of dietetic care and were familiar with nutritional support. However, almost $50 \%$ of the patients did not meet the recommended intake level of energy and 36\% of the patients were underweight based on their BMI $\left(\leq 18.5 \mathrm{~kg} / \mathrm{m}^{2}\right)$. There was no significant 
improvement in $\mathrm{BMI}$ or fat free mass index (FFMI) between waiting list entry and the last visit before LTX, although further deterioration was prevented. The number of patients who were underweight was reduced from $13(42 \%)$ to 8 (26\%). Of the nine patients who died before LTx, $67 \%$ were underweight and $78 \%$ had a low FFMI. Of the ten patients who died after LTx, $40 \%$ were underweight and $60 \%$ had a low FFMI. Forty-one patients, who survived LTX, had a somewhat better nutritional status: $34 \%$ were underweight and $40 \%$ had a low FFMI. After LTX, the body weight increased significantly compared to body weight before LTX. The mean weight gain at $18-24$ months post-LTX, as a proportion of initial body weight, was $11 \%$ for men and $8 \%$ for women. The largest increase in body weight was seen in men who were underweight at the start of the study. Our study was limited by its retrospective approach; not all data could be retrieved. For 12 CF LTx patients who died within one year after LTx no medical records were available. It would have been interesting to know their BMI and FFMI before LTX. Our future perspectives for research should focus on the impact of nutritional status on prognosis, on the specific nutritional requirements at several stages of the treatment process and on the effectiveness of several different nutritional support measures.

The aim for dieticians in transplant care is still to achieve an improvement or normalization of BMI and especially FFMI in pre-LTx candidates. The multidisciplinary treatment approach is becoming more important for pre-operative rehabilitation in patients. Early intervention and monitoring physical activity ${ }^{73}$ is warranted to achieve muscle mass and to increase the FFMI of patients. The prevention of organ rejection is an important goal in post-transplant care to achieve better survival outcomes. ${ }^{74}$ According to the most recent registry of the International Society for Heart and Lung Transplantation (ISHLT), CF is the third highest indication for LTX in adults and shows the best survival rate compared to patients with other diseases. The median survival time of CF recipients is 9.2 years. ${ }^{75}$ Unfortunately, comorbidities such as gastrointestinal malignancies (50) occur after LTX. The effects of the interaction between medication and food are already receiving attention ${ }^{76-78}$ and need to be explored in future studies. Moreover, comorbidities may increase as life expectancy of CF LTX survivors improves.

\section{CONCLUDING REMARKS AND FUTURE PERSPECTIVES}

The nutritional challenges in adults with CF have been explored, especially in patients before and after LTx. New drug therapies will improve the perspectives in life and the survival of CF individuals. It would be valuable to monitor the CF population, using this type of medication, for a longer period, particularly to find out if they are better able to preserve a good pulmonary 
function for a longer period of time and to reduce the necessity of having LTX. Additionally, BIA and REE measurements are becoming more important for diagnosing and monitoring dietetic interventions. In new (nutritional) research using intervention studies, these measurement tools can be applied. New challenges for specialized CF dieticians include the nutritional care in CF individuals of different ages and showing various disease conditions, such as overweight/ obesity ${ }^{11,19,79,80}$, CFRD ${ }^{81,82}$, renal failure ${ }^{83,84}$ and/or colon cancer before ${ }^{85,86}$ and after LTX. ${ }^{54,87}$ In concordance with medical and digital developments, the role of dieticians will evolve into a 'nutrition coach' for all CF individuals.

Perspectives for new research in CF patients (before and after LTX) are: CFTR modulators and the effect on pancreatic function and nutrient absorption; dietary intervention in CFRD and evaluation of body composition using BIA; eHealth in CF dietary intervention: impact on the nutritional status; and REE measurement in LTX candidates at the ICU unit. New research questions can be generated and will offer new opportunities to improve dietetic interventions and outcomes in the current CF population. 


\section{REFERENCES}

1. Elborn JS, Bell SC, Madge SL, et al. Report of the European Respiratory Society/European Cystic Fibrosis Society task force on the care of adults with cystic fibrosis. Eur Respir J. 2016;47:420-8.

2. Stephenson AL, Sykes J, Berthiaume Y, et al. Clinical and demographic factors associated with post-lung transplantation survival in individuals with cystic fibrosis. J Heart Lung Transplant. 2015;34:1139-45.

3. Stephenson AL, Mannik LA, Walsh S, et al. Longitudinal trends in nutritional status and the relation between lung function and BMI in cystic fibrosis: a population-based cohort study. Am J Clin Nutr. 2013;97:872-7.

4. Turck D, Braegger CP, Colombo C, et al. ESPEN-ESPGHAN-ECFS guidelines on nutrition care for infants, children, and adults with cystic fibrosis. Clin Nutr. 2016;35:557-77.

5. van der Haak N, King SJ, Crowder T, et al. Highlights from the nutrition guidelines for cystic fibrosis in Australia and New Zealand. J Cystic Fibros. 2019.

6. Mariani AW and Pego-Fernandes PM. Observational studies: why are they so important? Sao Paulo Med J. 2014;132:1-2.

7. Ligthelm RJ, Borzi V, Gumprecht J, Kawamori R, Wenying Y, Valensi P. Importance of observational studies in clinical practice. Clin Ther. 2007;29 Spec No:1284-92.

8. Shimmin D, Lowdon J, Remmington T. Enteral tube feeding for cystic fibrosis. Cochrane Database Syst Rev. 2019;7:CD001198.

9. Schwarzenberg SJ, Hempstead SE, McDonald CM, et al. Enteral tube feeding for individuals with cystic fibrosis: Cystic Fibrosis Foundation evidence-informed guidelines. J Cystic Fibrosis. 2016;15:724-35.

10. WoestenenkJW, Castelijns SJAM, van der Ent CK, Houwen RHJ. Nutritional intervention in patients with Cystic Fibrosis: A systematic review. J Cystic Fibros. 2013;12:102-15.

11. Kapnadak SG, Ramos KJ, Lopriore AM, Goss CH, Aitken ML. A Survey Identifying Nutritional Needs in a Contemporary Adult Cystic Fibrosis Cohort. BMC Nutr. 2019;5.

12. Iskrov GG, Stefanov RS, Lopez-Bastida J, et al. Economic Burden And Health-Related Quality Of Life Of Patients With Cystic Fibrosis In Bulgaria. Folia Med (Plovdiv). 2015; 57: 56-64.

13. Bell SC, Mall MA, Gutierrez $\mathrm{H}$, et al. The future of cystic fibrosis care: a global perspective. Lancet Respir Med. 2019.

14. Borowitz D, Lubarsky B, Wilschanski M, et al. Nutritional Status Improved in Cystic Fibrosis Patients with the G551D Mutation After Treatment with Ivacaftor. Dig Dis Sci. 2016;61:198-207.

15. Rosenfeld M, Cunningham S, Harris WT, et al. An open-label extension study of ivacaftor in children with CF and a CFTR gating mutation initiating treatment at age 2-5years (KLIMB). J Cystic Fibros. 2019.

16. Heijerman HGM, McKone EF, Downey DG, et al. Efficacy and safety of the elexacaftor plus tezacaftor plus ivacaftor combination regimen in people with cystic fibrosis homozygous for the F508del mutation: a double-blind, randomised, phase 3 trial. Lancet. 2019.

17. Engelen MP, Com G, Deutz NE. Protein is an important but undervalued macronutrient in the nutritional care of patients with cystic fibrosis. Curr Opin Clin Nutr Metab Care. 2014;17:515-20. 
18. Gabler G, Coenen M, Lycett D, Stamm T. Towards a standardized nutrition and dietetics terminology for clinical practice: An Austrian multicenter clinical documentation analysis based on the International Classification of Functioning, Disability and Health (ICF)-Dietetics. Clin Nutr. 2019;38:791-9.

19. Alvarez JA, Ziegler TR, Millson EC, Stecenko AA. Body composition and lung function in cystic fibrosis and their association with adiposity and normal-weight obesity. Nutrition. 2016;32:447-52.

20. Smyth RL, Rayner O. Oral calorie supplements for cystic fibrosis. Cochrane Database Syst Rev. 2017;5:CD000406.

21. McColley SA. A safety evaluation of ivacaftor for the treatment of cystic fibrosis. Expert Opin Drug Saf. 2016;15:709-15.

22. Konstan MW, McKone EF, Moss RB, et al. Assessment of safety and efficacy of long-term treatment with combination lumacaftor and ivacaftor therapy in patients with cystic fibrosis homozygous for the F508del-CFTR mutation (PROGRESS): a phase 3, extension study. Lancet Respir Med. 2017;5:10718.

23. Sainath NN, Schall J, Bertolaso C, McAnlis C, Stallings VA. Italian and North American dietary intake after ivacaftor treatment for Cystic Fibrosis Gating Mutations. J Cystic Fibros. 2019;18:135-43.

24. Stark LJ, Opipari-Arrigan L, Filigno SS, et al. Web-Based Intervention for Nutritional Management in Cystic Fibrosis: Development, Usability, and Pilot Trial.J Pediatr Psychol. 2016;41:510-21.

25. Hilty DM, Chan S, Hwang T, Wong A, Bauer AM. Advances in mobile mental health: opportunities and implications for the spectrum of e-mental health services. Mhealth. 2017;3:34.

26. Talboom-Kamp EP, Verdijk NA, Kasteleyn MJ, et al. High Level of Integration in Integrated Disease Management Leads to Higher Usage in the e-Vita Study: Self-Management of Chronic Obstructive Pulmonary Disease With Web-Based Platforms in a Parallel Cohort Design. J Med Internet Res. 2017;19:e185.

27. Martinez-Millana A, Zettl A, Floch J, et al. The Potential of Self-Management mHealth for Pediatric Cystic Fibrosis: Mixed-Methods Study for Health Care and App Assessment. JMIR Mhealth Uhealth. 2019;7:e13362.

28. Harris JA, Benedict FG. A Biometric Study of Human Basal Metabolism. Proc Natl Acad Sci U S A. 1918;4:370-3.

29. Schofield WN. Predicting basal metabolic rate, new standards and review of previous work. Hum Nutr Clin Nutr. 1985;39 Suppl 1: 5-41.

30. Energy and protein requirements. Report of a joint FAO/WHO/UNU Expert Consultation. World Health Organ Tech Rep Ser. 1985;724:1-206.

31. Blasco Redondo R. Resting energy expenditure; assessment methods and applications. Nutr Hosp. 2015;31Suppl 3: 245-54.

32. Psota T, Chen KY. Measuring energy expenditure in clinical populations: rewards and challenges. EurJ Clin Nutr. 2013;67:436-42.

33. Moudiou T, Galli-Tsinopoulou A, Vamvakoudis E, Nousia-Arvanitakis S. Resting energy expenditure in cystic fibrosis as an indicator of disease severity. J Cystic Fibrosis. 2007;6:131-6.

34. Mtaweh H, Tuira L, Floh AA, Parshuram CS. Indirect Calorimetry: History, Technology, and Application. Frontiers in Pediatrics. 2018;6.

35. Perez-Suarez I, Martin-Rincon M, Gonzalez-Henriquez JJ, et al. Accuracy and Precision of the COSMED K5 Portable Analyser. Front Physiol. 2018;9:1764. 
36. Henes ST, Johnson A, Toner M, et al. Assessing Resting Metabolic Rate in Overweight and Obese Adolescents With a Portable Indirect Calorimeter: A Pilot Study for Validation and Reliability. Nutr Clin Pract. 2016;31:355-61.

37. Yatabe T. Strategies for optimal calorie administration in critically ill patients. J Intensive Care. 2019;7.

38. Sabatino A, Theilla M, Hellerman M, et al. Energy and Protein in Critically III Patients with AKI: A Prospective, Multicenter Observational Study Using Indirect Calorimetry and Protein Catabolic Rate. Nutrients. 2017;9.

39. Singer P. Preserving the quality of life: nutrition in the ICU. Crit Care. 2019;23.

40. Bosy-Westphal A, Muller MJ. Identification of skeletal muscle mass depletion across age and BMI groups in health and disease--there is need for a unified definition. International journal of obesity. 2015;39:379-86.

41. Kyle UG, Genton L, Karsegard L, Slosman DO, Pichard C. Single prediction equation for bioelectrical impedance analysis in adults aged 20--94 years. Nutrition. 2001;17:248-53.

42. Earthman CP. Body Composition Tools for Assessment of Adult Malnutrition at the Bedside: A Tutorial on Research Considerations and Clinical Applications. JPEN Journal of parenteral and enteral nutrition. 2015;39:787-822.

43. Charatsi AM, Dusser P, Freund R, et al. Bioelectrical impedance in young patients with cystic fibrosis: Validation of a specific equation and clinical relevance. J Cystic Fibrosis. 2016;15:825-33.

44. Ceniccola GD, Castro MG, Piovacari SMF, et al. Current technologies in body composition assessment: advantages and disadvantages. Nutrition. 2019;62:25-31.

45. Sheean P, Gonzalez MC, Prado CM, McKeever L, Hall AM, Braunschweig CA. American Society for Parenteral and Enteral Nutrition Clinical Guidelines: The Validity of Body Composition Assessment in Clinical Populations. JPEN Journal of parenteral and enteral nutrition. 2019.

46. Tosato M, Marzetti E, Cesari M, et al. Measurement of muscle mass in sarcopenia: from imaging to biochemical markers. Aging Clin Exp Res. 2017;29:19-27.

47. Weill D, Benden C, Corris PA, et al. A consensus document for the selection of lung transplant candidates: 2014--an update from the Pulmonary Transplantation Council of the International Society for Heart and Lung Transplantation. J Heart Lung Transplant. 2015;34:1-15.

48. Sullivan JS and Mascarenhas MR. Nutrition: Prevention and management of nutritional failure in Cystic Fibrosis. J Cystic Fibrosis. 2017;16Suppl 2:S87-S93.

49. Hollander FM, Kok A, de Roos NM, Belle-van Meerkerk G, van de Graaf EA. Prediction Equations Underestimate Resting Energy Expenditure in Patients With End-Stage Cystic Fibrosis. Nutr Clin Pract. 2017;32:116-21.

50. Kalnins D, Pencharz PB, Grasemann H, Solomon M. Energy expenditure and nutritional status in pediatric patients before and after lung transplantation. J Pediatr. 2013;163:1500-2.

51. Jomphe V, Lands LC, Mailhot G. Nutritional Requirements of Lung Transplant Recipients: Challenges and Considerations. Nutrients. 2018;10.

52. Roza AM, Shizgal HM. The Harris Benedict equation reevaluated: resting energy requirements and the body cell mass. Am J Clin Nutr. 1984;40:168-82.

53. Belle-van Meerkerk G, van de Graaf EA, Kwakkel-van Erp JM, et al. Diabetes before and after lung transplantation in patients with cystic fibrosis and other lung diseases. Diabet Med. 2012;29:e15962. 
54. Li SS, Tumin D, Krone KA, et al. Risks associated with lung transplantation in cystic fibrosis patients. Expert Rev Respir Med. 2018;12:893-904.

55. Plasqui G, Westerterp KR. Physical activity assessment with accelerometers: an evaluation against doubly labeled water. Obesity. 2007;15:2371-9.

56. Vaquero Barrios JM, Redel Montero J, Santos Luna F. Comorbidities impacting on prognosis after lung transplant. Arch Bronconeumol. 2014;50:25-33.

57. Savioli G, Surbone S, Giovi I, et al. Early development of metabolic syndrome in patients subjected to lung transplantation. Clin Transplant. 2013;27:E237-43.

58. Conway SP, Morton A, Wolfe S. Enteral tube feeding for cystic fibrosis. Cochrane Database Syst Rev. 2008:CD001198.

59. Perona F, Castellazzi G, De luliis A, Rizzo L. Percutaneous radiologic gastrostomy: a 12-year series. Gut Liver. 2010;4Suppl 1:S44-9.

60. Ledder O, Oliver MR, Heine RG, Graham J, Volders E, Robinson PJ. Clinical audit results in earlier nutritional intervention in malnourished children with cystic fibrosis with improved outcome. $J$ Paediatr Child Health. 2015;51:988-93.

61. Stallings VA, Stark LJ, Robinson KA, et al. Evidence-based practice recommendations for nutritionrelated management of children and adults with cystic fibrosis and pancreatic insufficiency: results of a systematic review. J Am Diet Assoc. 2008;108:832-9.

62. Quintana-Gallego E, Ruiz-Ramos M, Delgado-Pecellin I, Calero C, Soriano JB, Lopez-Campos JL. Mortality from cystic fibrosis in Europe: 1994-2010. Pediatr Pulmonol. 2016;51:133-42.

63. Harness-Brumley CL, Elliott AC, Rosenbluth DB, Raghavan D, Jain R. Gender differences in outcomes of patients with cystic fibrosis. J Womens Health (Larchmt). 2014;23:1012-20.

64. Morton A and Wolfe S. Enteral tube feeding for cystic fibrosis. Cochrane Database Syst Rev. 2015: CD001198.

65. Sanz-Paris A, Alvarez Hernandez J, Ballesteros-Pomar MD, et al. Evidence-based recommendations and expert consensus on enteral nutrition in the adult patient with diabetes mellitus or hyperglycemia. Nutrition. 2017;41:58-67.

66. Nicolo M, Stratton KW, Rooney W, Boullata J. Pancreatic enzyme replacement therapy for enterally fed patients with cystic fibrosis. Nutr Clin Pract. 2013;28:485-9.

67. Freedman SD. Options for addressing exocrine pancreatic insufficiency in patients receiving enteral nutrition supplementation. Am J Manag Care. 2017;23:S220-S8.

68. Bell SC, Bowerman AR, Davies CA, Campbell IA, Shale DJ, Elborn JS. Nutrition in adults with cystic fibrosis. Clin Nutr. 1998;17:211-5.

69. Dray X, Kanaan R, Bienvenu T, et al. Malnutrition in adults with cystic fibrosis. Eur J Clin Nutr. 2005;59:152-4.

70. Madill J, Gutierrez C, Grossman J, et al. Nutritional assessment of the lung transplant patient: body mass index as a predictor of 90-day mortality following transplantation.J Heart Lung Transplant. 2001;20:288-96.

71. Esteves GB, Macedo A, Nakasato M, Bruno ML. Evolution of nutritional status in lung transplant candidates who are initially malnourished or overweight. J Bras Pneumol. 2009;35:1217-9.

72. Forli L, Pedersen JI, Bjortuft O, Vatn M, Boe J. Dietary support to underweight patients with endstage pulmonary disease assessed for lung transplantation. Respiration. 2001;68:51-7. 
73. Wickerson L, Rozenberg D, Janaudis-Ferreira T, et al. Physical rehabilitation for lung transplant candidates and recipients: An evidence-informed clinical approach. World J Transplant. 2016;6:51731.

74. Adegunsoye A, Strek ME, Garrity E, Guzy R, Bag R. Comprehensive Care of the Lung Transplant Patient. Chest. 2017;152:150-64.

75. Yusen RD, Edwards LB, Dipchand Al, et al. The Registry of the International Society for Heart and Lung Transplantation: Thirty-third Adult Lung and Heart-Lung Transplant Report-2016; Focus Theme: Primary Diagnostic Indications for Transplant. J Heart Lung Transplant. 2016;35:1170-84.

76. Sridharan K, Sivaramakrishnan G. Interaction of Citrus Juices with Cyclosporine: Systematic Review and Meta-Analysis. EurJ Drug Metab Pharmacokinet. 2016;41:665-73.

77. Asfaw M, Mingle J, Hendricks J, Pharis M, Nucci AM. Nutrition management after pediatric solid organ transplantation. Nutr Clin Pract. 2014;29:192-200.

78. Mooij SH, Hogema BM, Tulen AD, et al. Risk factors for hepatitis E virus seropositivity in Dutch blood donors. BMC Infect Dis. 2018;18:173.

79. Hanna RM, Weiner DJ. Overweight and obesity in patients with cystic fibrosis: a center-based analysis. Pediatr Pulmonol. 2015;50:35-41.

80. Panagopoulou P, Fotoulaki M, Nikolaou A, Nousia-Arvanitakis S. Prevalence of malnutrition and obesity among cystic fibrosis patients. Pediatr Int. 2014;56:89-94.

81. Frost F, Dyce $P$, Ochota A, et al. Cystic fibrosis-related diabetes: optimizing care with a multidisciplinary approach. Diabetes Metab Syndr Obes. 2019;12:545-52.

82. Birch L, Lithander FE, Hewer SL, Harriman K, Hamilton-Shield J, Perry R. Dietary interventions for managing glucose abnormalities in cystic fibrosis: a systematic review protocol. Syst Rev. 2018;7:98.

83. Quon BS, Mayer-Hamblett N, Aitken ML, Goss CH. Risk of post-lung transplant renal dysfunction in adults with cystic fibrosis. Chest. 2012;142:185-91.

84. Jardel S, Reynaud Q, Durieu I. Long-term extrapulmonary comorbidities after lung transplantation in cystic fibrosis: Update of specificities. Clin Transplant. 2018;32:e13269.

85. Hadjiliadis D, Khoruts A, Zauber AG, et al. Cystic Fibrosis Colorectal Cancer Screening Consensus Recommendations. Gastroenterology. 2018;154:736-45 e14.

86. Abraham JM, Taylor CJ. Cystic Fibrosis \& disorders of the large intestine: DIOS, constipation, and colorectal cancer. J Cystic Fibrosis. 2017;16Suppl 2:S40-S9.

87. Yamada A, Komaki Y, Komaki F, Micic D, Zullow S, Sakuraba A. Risk of gastrointestinal cancers in patients with cystic fibrosis: a systematic review and meta-analysis. Lancet Oncol. 2018;19:758-67. 
蛋

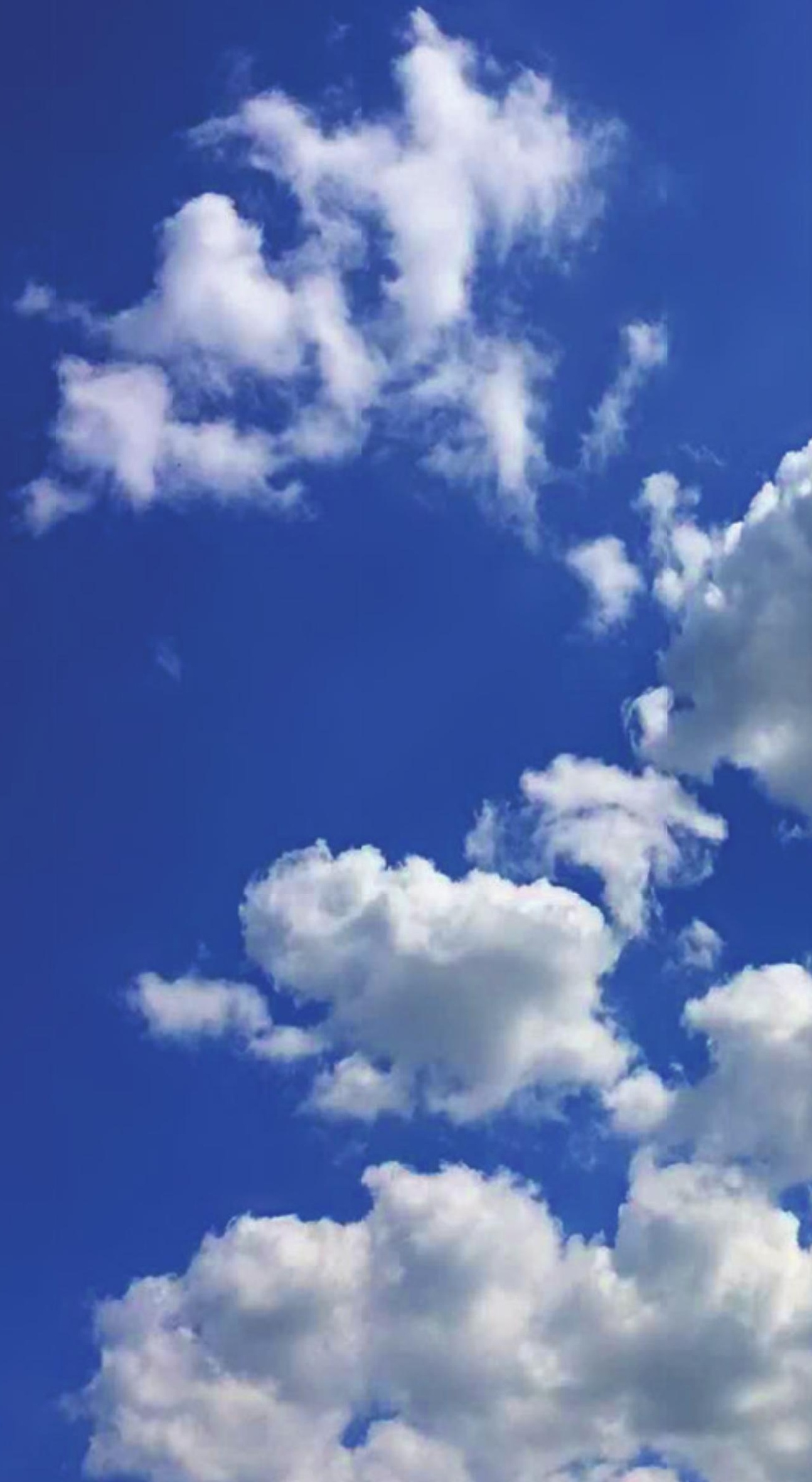




\section{Chapter 9}

Summary in Dutch - Nederlandse samenvatting Acknowledgement - Dankwoord Curriculum Vitae 


\section{SUMMARY IN DUTCH - NEDERLANDSE SAMENVATTING}

Cystic fibrosis (CF) is een van de meest voorkomende erfelijke en ongeneeslijke aandoeningen wereldwijd. Het wordt veroorzaakt door een afwijking in het 'cystic fibrosis transmembrane conductance regulator'(CFTR)-gen. Dit gen codeert voor het CFTR-eiwit, dat dient als een kanaal voor chloride-ionen en water door het membraan van de cellen die slijm, zweet, speeksel, tranen en spijsverteringsenzymen produceren. Een afwijking in dit CFTR-gen schaadt de functie van deze kanalen en leidt tot dik en taai slijm. Omdat het CFTR-eiwit aanwezig is in alle slijmproducerende organen in het lichaam, is CF een genetische multisysteemaandoening die de longen, alvleesklier, lever, darmen en andere organen aantast. Symptomen van CF kunnen variëren door de vele verschillende afwijkingen in het CFTR-gen. Mensen met CF kunnen problemen hebben met de opname van vet uit de voeding en last hebben van chronische longinfecties. Deze problemen kunnen leiden tot verslechtering van de voedingsstatus en progressieve longschade.

De behandeling richt zich op het handhaven en het optimaliseren van de longfunctie en de voedingsstatus, omdat dit sleutelfactoren zijn voor de overleving. Een grote verbetering in overleving van mensen met CF is in de afgelopen decennia aangetoond. In West-Europa, de Verenigde Staten en Canada is de mediane overleving voor mensen met CF ongeveer 40-50 jaar. Vanwege ongelijkheden op het gebied van beschikbare behandelingen is de gemiddelde overleving van mensen met CF in heel Europa ongeveer slechts 30 jaar. De toegenomen overleving kan worden verklaard door een combinatie van het gebruik van screeningsprogramma's bij pasgeborenen, gecentraliseerde zorg gebruikmakend van evidence-based klinische behandelings- en voedingsrichtlijnen, de bredere toegang tot CFspecifieke medicijnen en de introductie van nieuwe medicijnen die CFTR-eiwit moduleren. Het aantal volwassenen met CF groeit, aangezien de meerderheid van de kinderen met CF nu volwassen wordt. Dit betekent ook dat meer mensen met CF verschillende stadia van longziekte hebben, vaak met complexe comorbiditeit.

Een goede voedingsstatus draagt bij aan een betere longfunctie en overleving bij CF. De kans op een verminderde voedingsstatus bij CF is echter groot en is het gevolg van een combinatie van verhoogde voedingsbehoeften, verhoogde verliezen van voedingsstoffen en verminderde voedingsinname. Het is vaak moeilijk voor iemand met CF om een goede voedingsstatus te bereiken en te behouden. Oorzaken hiervoor zijn divers en worden mede veroorzaakt door CF-gerelateerde klachten zoals benauwdheid, hoesten, het opgeven van slijm, koorts, buikpijn, darmverstopping, vermoeidheid en terugkerende luchtweginfecties. Door infecties in de 
Iongen ontstaat vaak een verminderde eetlust. Er kan snel een negatieve cirkel ontstaan. Door de verminderde eetlust treedt er een tekort aan energie en voedingsstoffen op dat tot gevolg heeft dat de weerstand vermindert. Door een verminderde weerstand neemt de gevoeligheid voor longinfecties toe en de longinfecties veroorzaken een verminderde eetlust.

Voor mensen met CF bestaan specifieke voedingsrichtlijnen die adviseren $110-200 \%$ van de benodigde energie te gebruiken ten opzichte van gezonde leeftijdsgenoten van hetzelfde geslacht. Hiermee kunnen de streefwaarden voor een goede voedingsstatus, uitgedrukt in body mass index (BMI), bij CF worden bereikt en gehandhaafd. Als streefwaarde bij CF geldt een BMI op of boven de $50^{e}$ percentiel bij kinderen en voor volwassenen op of boven $22 \mathrm{~kg} / \mathrm{m}^{2}$ voor vrouwen en $23 \mathrm{~kg} / \mathrm{m}^{2}$ voor mannen. Een goede BMI of normaal gewicht voor leeftijd en geslacht kan echter voedingsproblemen maskeren, omdat deze maten geen inzicht geven in de hoeveelheid vetmassa (VM) en vetvrije massa (VVM). Inzicht in deze lichaamssamenstelling is belangrijk, omdat mensen met CF en hogere VVM een betere longfunctie blijken te hebben dan mensen met CF en hogere VM. Bovendien is een afname van of lage VVM geassocieerd met een verminderde longfunctie. Het verlies van zowel de spiermassa (aanwezig in de VVM) als de spierfunctie moet worden voorkomen, omdat dit leidt tot ongewenst gewichtsverlies, uitputting en verminderde fysieke activiteit, hetgeen weer bijdraagt aan een hoger risico op overlijden. Als onvoldoende voedingsinname plaatsvindt, is beschreven dat voeding toegediend via een sonde in de maag of darm een effectieve methode is om de voedingsstatus te verbeteren, vooral bij ondervoede mensen.

Bij mensen met CF komt steeds meer overgewicht en obesitas voor. Gewichtsmetingen en BMI alleen detecteren hierbij geen veranderingen in de lichaamssamenstelling. De metingen van lichaamssamenstelling kunnen de resultaten van voedingsinterventies beter zichtbaar maken.

Ondanks betere behandelingsmethoden op het gebied van medicatie en voeding, hebben de meeste mensen met CF nog steeds een longtransplantatie (LTX) nodig om de overleving te verbeteren. Deze LTx moet worden overwogen voor mensen bij wie de klinische status ondanks maximale medische behandeling geleidelijk is afgenomen. Verwijzing naar een longtransplantatiecentrum is noodzakelijk als de longfunctie (uitgedrukt in FEV1) tot beneden de $30 \%$ van de voorspelde waarde van een gezonde leeftijdsgenoot van hetzelfde geslacht is gedaald of als de longfunctie een snel dalende FEV1 laat zien ondanks optimale behandeling. Als geen contra-indicaties zijn, worden de mensen vervolgens op de wachtlijst voor LTX geplaatst. 
Om de optimale overlevingskansen voor en na LTx te bereiken, is in Nederland in 2014 de LAS (Long Allocatie Score) geïntroduceerd. De LAS wordt gebruikt als maat voor de ernst van de ziekte met scores variërend van 0 tot 100. Een hoge LAS reflecteert mensen die een LTx het meest dringend nodig hebben. Voor de huidige LAS-berekening zijn antropometrische parameters zoals gewicht en lengte nodig. Aanhoudend gewichtsverlies ondanks optimale inzet van voedingstherapie is één van de huidige criteria voor mensen om in aanmerking te komen voor LTx. Relatieve contra-indicaties voor LTx zijn echter een voedingsstatus met een BMI lager dan 18 of hoger dan 30. Het is een uitdaging voor diëtisten die in LTX-zorg werken om de voedingstoestand bij mensen met CF voor LTx te verbeteren. Een belangrijk onderdeel hierbij is het vaststellen van de benodigde energiebehoefte. Indirecte calorimetrie (IC) is de meest gebruikte en betrouwbare niet-invasieve methode voor het meten van het energieverbruik in rust (REE) in klinische populaties, ook bij CF. Een onderzoek uitgevoerd bij 10 kinderen met CF toonde een verhoogd (132\% van voorspeld via voorspellingsformules) gemeten REE door IC voor LTx. Binnen een jaar na LTx daalde dit tot $112 \%$ van de voorspelde REE. Langdurige studies waarin REE voor en na LTX wordt onderzocht bij volwassenen met CF ontbreken echter

Bij mensen met CF voor en na het LTx-traject is het van belang om te onderzoeken of de $\mathrm{BMI}$ een goede maat is om de voedingstoestand te beoordelen. In de huidige internationale richtlijnen wordt dit tot nu toe aanbevolen. Ook moeten huidige diagnostische hulpmiddelen voor de beoordeling van de voedingsstatus en energiebehoefte bij CF worden onderzocht alsmede de toepasbaarheid van deze hulpmiddelen bij LTx. Verder is onderzoek naar effectieve voedingsinterventiestrategieën bij mensen met CF en een LTx zeldzaam.

Dit proefschrift richt zich op het vergroten van de kennis over en het verbeteren van het meten van de lichaamssamenstelling, de voedingsbehoeften en de effectiviteit van voedingsinterventies bij mensen met CF. Dit is essentieel voor betere voedingszorg 'op maat', rekening houdend met de verschillende stadia en complicaties van CF gedurende het leven om de overleving te verlengen en de kwaliteit van leven te verbeteren. Voor deze geïndividualiseerde voedingsbehandeling van mensen met CF is de kennis en de klinische ervaring van een gespecialiseerde CF-diëtist onontbeerlijk. We onderzoeken hoe het beste gemeten kan worden en hoe de resultaten gebruikt kunnen worden in de klinische praktijk. Gegevens hiervoor zijn verkregen uit de klinische praktijk, variërend van mensen die op de polikliniek regelmatig op controles kwamen tot mensen met eindstadium longziekte voor LTX en mensen met donorlongen. 
In Hoofdstuk 2 geven wij een overzicht van het nieuwste bewijs en de aanbevelingen voor de optimale voedingsbehandeling bij mensen met CF. In deze review werd ook de meest recente literatuur onderzocht met betrekking tot de positieve associatie tussen voedingsstatus met longfunctie en overleving. Sinds 2016 zijn diverse richtlijnen gepubliceerd om te komen tot een goede voedingsbehandeling bij CF. De standaard voedingszorg voor CF is een hoogcalorisch dieet met gebruik van enzymvervangende therapie (PERT) en vet oplosbare vitamine supplementen om een goede voedingsstatus te bereiken en te behouden. De voedingsstatus kan echter ook worden verbeterd door CFTR-modulatoren te gebruiken, inclusief correctoren en potentiatoren. Deze verschillende medicamenteuze therapieën zijn nu beschikbaar in de klinische praktijk met positieve effecten op de voedingsstatus. De optimale behandeling voor iemand met CF kan worden bereikt en toegepast door persoonlijke medische behandeling, adequate voedingsinterventie en gezonde leefstijl te combineren. De CFTR-modulatie en de effecten ervan op de voedingsstatus en longfunctie zullen uitdagingen bieden om voedingsinterventies aan te passen in de huidige CF-zorg en in de nabije toekomst. Eén van de uitdagingen is het eetlust-verhogende effect van CFTR-modulatoren. Dit kan gemakkelijk leiden tot ongewenste gewichtstoename en een ongunstige toename van VM. Omdat een goede BMI met een te hoog percentage VM en ook obesitas bij mensen met CF vaker voorkomen, moeten wijzigingen in voedingsaanbevelingen worden doorgevoerd. Bij mensen met CF, die CFTR-modulatoren gebruiken, kan de meting van REE frequenter ingezet worden om veranderingen in het energiemetabolisme te onderzoeken. Enkele recente studies beschrijven een daling van REE bij gebruik van deze CFTR-modulatoren. Een nieuwe focus op het gebruik van een uitgebalanceerd dieet gecombineerd met een gezonde leefstijl en goede bewegingsadviezen is ook bij mensen met CF hard nodig om te streven naar een goede lichaamssamenstelling met normale vetmassa en vetvrije massaverhouding.

De huidige klinisch toepasbare methoden om lichaamssamenstelling en REE te bepalen, zijn onderzocht in diverse onderzoeken binnen onze groep mensen met CF. In Hoofdstuk 3 zijn de resultaten weergegeven van het onderzoek waarin we gemeten REE vergelijken met geschatte REE voorspeld door verschillende formules uit de klinische praktijk. We hebben dit onderzocht in een groep mensen met CF en eindstadium longziekte en in een groep mensen met CF na LTx. Onze studie was de eerste die heeft gekeken naar overeenkomsten tussen gemeten REE met behulp van IC en voorspelde REE volgens de voorspellingsformules van Harris-Benedict (1919 en 1984), Schofield en WHO (1985). Bovendien was deze studie de eerste die verschillen in REE heeft onderzocht voor VVM in een groep mensen met CF voor LTx en een groep mensen met CF na LTX. REE geschat door voorspellingsformules kwam overeen met gemeten REE bij maximaal $25 \%$ van de mensen met CF voor LTx en bij $67 \%$ van 
de mensen met CF na LTX. In onze onderzoekspopulatie werd de REE onderschat met behulp van voorspellingsformules bij alle mensen met CF en diabetes (CFRD) voor LTx, geïnfecteerd met de Pseudomonas aeruginosa ( $P$ aeruginosa) bacterie en met een homozygote $\Delta \mathrm{F} 508$ gen mutatie. Binnen 2 jaar na LTx werd bij 4 van de 12 getransplanteerde mensen met CF de REE nog steeds onderschat met de voorspellingsformules, mogelijk vanwege vernieuwde infectie met $P$ aeruginosa via de neusbijholten. REE berekend per kilogram lichaamsgewicht en REE per kilogram VVM waren significant hoger in de groep mensen met CF voor LTx dan in de groep mensen met CF na LTx. Deze verschillen kunnen worden verklaard door een afname van ontstekingen en longinfecties na LTx en een afname van de ademhalingsinspanning.

In Hoofdstuk 4 beschrijven we de bio-elektrische impedantieanalyse (BIA) als een methode om de lichaamssamenstelling bij volwassenen met CF vast te stellen. Ook onderzoeken we of de verschillen tussen nuchtere en niet-nuchtere BIA-resultaten klinisch relevant zijn. Uit onze studie blijkt dat voedings- en vochtinname de waarden van VVM met 0,23 kg verhogen en de waarden van VM met 0,22 kg verlagen. Bovendien was bij $86 \%$ van de mensen het verschil in waarden van VVM en VM na voedings- en vochtinname minder dan $1 \mathrm{~kg}$. De uitkomsten bleven bij 83\% van de patiënten gelijk nadat de VVM en VM ook voor lengte werd gecorrigeerd. De verschillen tussen nuchtere en niet-nuchtere VVM en VM waren niet klinisch relevant en de samenhang met longfunctie bleef gelijk. Voor het beoordelen en het evalueren van de voedingsstatus kunnen de BIA-metingen worden uitgevoerd na voedings- en vochtinname door volwassenen met CF die de polikliniek bezoeken. Deze resultaten geven ook aan dat niet-nuchtere BIA-metingen kunnen worden geregistreerd in elektronische patiëntendossiers en dat onderzoekers deze gegevens voor onderzoeksdoeleinden kunnen gebruiken. De onderzoeksresultaten moeten echter voorzichtig worden geïnterpreteerd wanneer ze worden toegepast op andere apparaten voor lichaamssamenstelling. De reproduceerbaarheid neemt toe wanneer de metingen in de loop van de tijd worden uitgevoerd onder vergelijkbare testomstandigheden.

Dezelfde technieken van BIA en REE zijn ook gebruikt in een unieke groep mensen met CF voor LTX in combinatie met vervolgmetingen tot en met 2 jaar na LTX. De resultaten van dit onderzoek zijn beschreven in Hoofdstuk 5. Onze studie toont aan dat REE en REE gecorrigeerd voor kilogram VVM aanzienlijk afnemen na LTx. De voorspellingsformules blijven onnauwkeurig tot een jaar na LTX. Vanaf een jaar na LTx blijven REE en REE per kilogram VVM stabiel en is de voorspelling van REE met behulp van formules nauwkeurig (d.w.z. binnen 10\% van de gemeten waarde) bij ongeveer tweederde van de mensen met CF. Om onderschatting van de energiebehoeften te voorkomen, raden we daarom aan om de gemeten REE met IC 
te gebruiken bij mensen met CF en eindstadium longziekte alsook bij mensen met CF tot een jaar na LTx. Na een jaar LTx kunnen de voorspellingsformules gebruikt worden in de klinische praktijk.

De effectiviteit van voedingsinterventies wordt bestudeerd in twee andere studies van ons en is beschreven in Hoofdstuk 6 en 7. De resultaten van een observationele studie, waarin de veranderingen in het lichaamsgewicht en de BMI voor en na het starten van sondevoeding (ETF) zijn onderzocht bij mensen met CF en eindstadium longziekte, worden beschreven in Hoofdstuk 6. De conclusie van onze studie is dat ETF het lichaamsgewicht en de BMI verbeterde. Gedurende 6 maanden na het starten van ETF is een gemiddelde toename in lichaamsgewicht van $3,5 \mathrm{~kg}$ en een $1,3 \mathrm{~kg} / \mathrm{m}^{2}$ toename in BMI te zien. De longfunctie was en bleef laag; voorspelde FEV1\% was 28\% in 6 maanden voor de start van ETF, 26\% bij de start van ETF en $25 \%$ in 6 maanden na de start van ETF. De klinische resultaten waren vergelijkbaar bij vrouwen en mannen, maar de steekproefgrootte voor alleen mannen was te klein om statistische significantie te bereiken.

In Hoofdstuk 7 onderzoeken we $\mathrm{BMI}$ en vetvrije massa-index (VVMI), voedingsinterventie en voedingsinname bij mensen met CF voor en na LTx. Van 75 mensen met CF hebben we gegevens kunnen gebruiken uit patiëntendossiers. Hiervan kregen 51 mensen met CF een LTx. Twee jaar volledige follow-upgegevens van het gewicht, de lichaamssamenstelling en de dieetinterventie waren beschikbaar voor 31 van deze 51 mensen, omdat 10 mensen overleden na LTx en 10 mensen minder dan 1 jaar gevolgd zijn. Bij de start van het LTx traject waren de meeste mensen met CF bekend met een lange geschiedenis van dieetbehandeling. Bijna $50 \%$ van de groep mensen kreeg echter niet hun behoefte aan energie binnen en $36 \%$ van de groep mensen was ondervoed op basis van hun BMI $\left(\leq 18,5 \mathrm{~kg} / \mathrm{m}^{2}\right)$. Significante verbetering in BMI of VVMI tijdens de wachtlijstperiode en het laatste bezoek voor transplantatie was niet aantoonbaar. Het aantal mensen met ondergewicht verminderde wel van $13(42 \%)$ tot 8 (26\%). Van de 9 patiënten die stierven voor LTx had $67 \%$ ondergewicht en $78 \%$ had een lage VVMI. Van de 10 patiënten die stierven na LTx had 40\% ondergewicht en 60\% een lage VVMI. Eenenveertig patiënten, die LTx overleefden, hadden een iets betere voedingsstatus: $34 \%$ had ondergewicht en 40\% had een lage VVMI. Na de LTX nam het lichaamsgewicht aanzienlijk toe in vergelijking met het lichaamsgewicht voor LTx. De gemiddelde gewichtstoename op 18-24 maanden na LTX, als percentage van het gewicht voor LTX, was $11 \%$ voor mannen en $8 \%$ voor vrouwen. De sterkste toename van het lichaamsgewicht werd waargenomen bij mannen met ondergewicht. 
In Hoofdstuk 8 zijn de resultaten van dit proefschrift bediscussieerd en samengevat. Voedingsproblemen bij volwassenen met CF zijn onderzocht, vooral bij mensen voor en na LTx. Nieuwe medicamenteuze therapieën, zoals de CFTR-modulatoren, zullen het perspectief in het leven en de overleving van mensen met CF verbeteren. Het is interessant om te onderzoeken of mensen met CF die dit type medicatie gebruiken, beter in staat zijn om een goede longfunctie te behouden tijdens hun leven en zich minder vaak zullen hoeven aanmelden voor een LTX.

Nieuwe uitdagingen op het gebied van CF-voedingszorg 'op maat' en CF-voedingsonderzoek kunnen uitgewerkt gaan worden voor en door gespecialiseerde CF-diëtisten. De voedingszorg blijft in ontwikkeling bij mensen met CF van verschillende leeftijden en bij diverse aandoeningen zoals overgewicht/obesitas, CFRD, nierfalen en/of darmkanker voor en na LTX. Hoe de diëtist als 'voedingscoach' diverse optimale voedingsbehandelingen motiveert en aan mensen met CF overbrengt, zal zich ook verder moeten ontwikkelen. Deze ontwikkeling heeft de grootste kans van slagen, indien dit gebeurt in combinatie met maximale participatie van de patiënt zelf en in samenhang met medische en digitale vernieuwingen.

Toekomstige onderzoeksvragen op het gebied van CF en voeding kunnen zich richten op verschillende aspecten van voedingsbehandelingen bij bovengenoemde aandoeningen bij CF. De uitkomsten van CF-gerelateerd voedingsonderzoek dragen bij aan de verbetering van dieetinterventies en -resultaten in de huidige CF populatie. Enkele voorbeelden van toekomstig noodzakelijk onderzoek zijn: onderzoek naar herstel van pancreasfunctie bij gebruik van CFTR modulatoren en effect op opname van voedingsstoffen; de rol van dieetinterventie bij CF gerelateerde diabetes en evaluatie van lichaamssamenstelling met behulp van BIA; de functie van eHealth in de diëtistische behandeling voor monitoring van de voedingsstatus; en REE onderzoek bij mensen met CF op de intensive care.

De voedingsstatus en optimale dieetinterventies blijven belangrijke aspecten gedurende het hele leven van mensen met CF. Het is belangrijk om BIA en REE metingen te gebruiken voor betere diagnostiek en monitoring van de geïndividualiseerde diëtistische zorg, alsook in te zetten bij voedingsinterventiestudies in CF. 
Summary in Dutch - Nederlandse samenvatting 


\section{ACKNOWLEDGEMENT - DANKWOORD}

Dit proefschrift is tot stand gekomen met de hulp van veel personen. Hierbij wil ik graag iedereen bedanken die betrokken is geweest met een aantal personen in het bijzonder.

Allereerst wil ik alle patiënten met cystic fibrosis danken voor hun inspirerende contacten tijdens poliklinische of klinische consulten. Mede door hun kritische vragen over voeding en voedingsgerelateerde onderwerpen zijn diverse onderzoeksvragen gegenereerd. Voedingsinterventie te onderbouwen vanuit wetenschappelijk oogpunt en het vertalen van resultaten naar de (poli)klinische patiëntenzorg is voor mij een hele belangrijke drijfveer geweest om de afgelopen jaren met veel enthousiasme onderzoek te doen. Dank voor jullie meedenken en inzet. Arian en Jarda, jullie in het bijzonder! Laat onze goede contacten een inspiratiebron blijven voor nieuw onderzoek in de nabije toekomst.

Geachte promotor, beste Harry, je hebt mij de afgelopen jaren de mogelijkheid geboden om onderzoek voort te zetten. Toen je in het UMCU kwam werken had je voor mij al een voorbeeldfunctie op het gebied van CF zorg. Op landelijke en internationale congressen was je enthousiasme duidelijk te zien en ik vond het heel bijzonder nu met je te mogen samenwerken in het UMCU. Dat jezelf een proefschrift geschreven hebt met de titel 'Studies in the Clinical Management of Cystic Fibrosis' vond ik heel leuk om te lezen. Hierin beschreef je als longarts al je aandacht voor voedingsresearch in CF! Je gedrevenheid, duidelijkheid en betrokkenheid bij patiënten en in onderzoek is een inspiratiebron voor mij geweest dit proefschrift te mogen afronden. In de gesprekken die we samen gevoerd hebben werd duidelijk wat goed geschreven was en wat niet. De publicaties zijn daarna snel tot stand gekomen. Jouw vertrouwen in mij dat het proefschrift snel af zou komen werkte heel stimulerend.

Geachte copromotor, beste Nicole, vele jaren werken we al samen. Vanaf het moment dat je bij onze afdeling Diëtetiek als stafmedewerker Onderwijs \& Onderzoek onder bezielend oog van Prof. Wija van Staveren werkzaam was, hebben we elkaar geïnspireerd om de klinische diëtetiek te combineren met wetenschappelijk onderzoek. Een samenwerking, die nadat je terugging naar de WUR in de functie van docent en onderzoeker voeding bij ziekte, altijd is gebleven en elkaar nog steeds versterkt. Wat heb ik veel van je geleerd! Je rust, creativiteit, positieve kritische feedback, wetenschappelijk blik en kennis hebben mij enorm verrijkt en kracht gegeven dit proefschrift te voltooien. Onze coaching van WUR studenten hierbij heeft ook onze samenwerking tot de beste uitkomsten gebracht, en laat zien dat $1+1$ niet 2 hoeft te zijn, maar in ons geval 3 . Het allerbeste resultaat bij optimale inzet. 
Ook een bijzonder woord van dank aan Ed van de Graaf. Als longtransplantatie/CF arts heb je mij gestimuleerd om met een promotietraject te beginnen en uren voor onderzoek via Professor Jan-Willem Lammers te genereren. Je empathie en je grote hart voor je patiënten en betrokkenheid bij mij als diëtist/onderzoeker (in de zorg en het onderzoek bij longtransplantatie) maakten dat we het 'onderzoeksvuur' konden ontsteken en gaande houden. En dit heeft geleid tot mooie artikelen rondom longtransplantatie en CF.

Geachte leden van de beoordelingscommissie, Prof. dr. C.K. van der Ent, prof. dr. R.H.J. Houwen, prof. dr. A.M.W.J. Schols, prof. dr. P.J.M. Weijs, prof. dr. J.C. Grutters, vriendelijk dank voor de tijd en aandacht die u allen heeft besteed aan het beoordelen van dit proefschrift. Beste Kors, een speciaal woord van dank aan jou, je prikkelende vragen hebben bijgedragen aan optimale voorbereiding en uitvoering van onderzoek. Ook jij hebt mij gestimuleerd het promotietraject te volgen, na vele jaren samenwerking binnen CF zorg en onderzoek, is het nu echt 'tijd voor een feestje'!

Beste medeauteurs Annemieke, Gerdien, Marcella, Jolein en studenten Yael, Anne, Denise, hartelijke bedankt voor jullie betrokkenheid bij de totstandkoming van de artikelen. leder met jullie eigen kracht en bijdrage maakte het schrijven van artikelen tot een mooie uitdaging met goede resultaten.

Beste Marleen de Bruin, als leidinggevende van onze afdeling Diëtetiek in het UMCU wil ik je heel erg bedanken voor de mogelijkheid om middelen en tijd te investeren in de uitvoering van mijn onderzoeken. Het aandachtsgebied Nutritional Assessment binnen onze afdeling is duidelijk geprofileerd in de publicaties van dit proefschrift. Het is ook heel fijn om te weten dat je trots bent op dit eindresultaat. Dat het een inspiratiebron mag zijn voor nieuw ambitieuze collega's Diëtetiek!

Beste teamleden CF, dank jullie wel voor de vele jaren van intensieve en prettige samenwerking. Met elkaar staan we als multidisciplinair team 'op de kaart' in CF Nederland. Ook de inspirerende congressen op het gebied van CF brengen behalve veel overdracht van kennis en kunde ook persoonlijke aandacht en betrokkenheid met zich mee. Dank voor jullie support Bert, Erik, Karin, Regina, Inez, Jeffrey, Hetty, Annelotte, Gitte, Peter, Myriam, Marian, Suzan, Cora, Sabine en Marit tijdens deelnames en presentaties op Europese congressen! Zo waardevol om als één mooi team te werken voor onze patiënten! Ook speciaal dank aan Wytze en Marcella; afstemming tussen fysiotherapie en diëtetiek maakt ons paramedisch werken met een academische onderbouwing nog uitdagender. Annet, jouw belangstelling 
en betrokkenheid voor de mijn onderzoeken voelt altijd heel prettig en waardevol. En ook Willeke en Cindy, bedankt voor het soepel verlopen van de uitvoeringen van de BIA metingen op de wekelijkse CF check up, hierdoor geen 'missing' data!

Beste teamleden longtransplantatie, ook vanuit dit multidisciplinaire team wordt er gewerkt aan optimale zorg voor deze groep kwetsbare patiënten. De gedrevenheid, passie en inzet van een ieder werkt aanstekelijk. Ben heel blij ook van dit team deel te mogen uitmaken en wat was het een prachtige ervaring samen met Marion de LWTV-NTV Innovatie-Kwaliteitsprijs te mogen ontvangen op het BOOT congres voor het gezamenlijk onderzoek getiteld 'Voedingssupplementen na longtransplantatie: advies of verbod?'. Heel veel succes met jullie promotietraject Dieuwertje en Marion! Het doorzetten, volhouden en wachten op acceptatie van een artikel, we hebben veel mogen delen! Ook de positieve feedback van Dagmar op het verzorgen van (Engelstalige) presentaties vergeet ik niet meer. En ook hierbij dank aan degenen die hebben bijgedragen dat logistiek alles goed kon verlopen. Marja, Sylvia en CeesJan, dank voor het samen puzzelen en inplannen van alle REE metingen!

Beste collega's Diëtetiek, intern en extern, bedankt voor de inzet in afgelopen jaren om patiëntenzorg over te nemen als ik bezig was met artikelen schrijven, presentaties te geven op (inter)nationale congressen of scholing te volgen om mijn academische kennis te vergroten. Het 'gedeeltelijk' los laten van patiëntenzorg was moeilijk in het begin maar gaandeweg lukte dit beter. Met vertrouwen heb ik de patiënten aan jullie goede zorg kunnen overdragen, vooral Jolanda, Nicole, Janine, Emma, Patty en Cora, veel dank voor jullie hulp hierbij! En ook het secretariaat Diëtetiek bemand door Moniek en Jeanette, dank voor het ondersteunen bij het inplannen van alle poliafspraken van patiënten die voor BIA en/of REE metingen kwamen. Ook mijn directe collega's van het Nutritional Assessment lab, Elles, Annemieke, Anne en Sytske, dank voor jullie kritische opmerkingen en inspirerende discussies om onderzoek op het gebied van Nutritional Assessment goed uit te voeren! We hanteren met elkaar: opgeven is geen optie! En Elles, dank voor de vele kopjes koffie, ze waren nodig en dat het soms behelpen is met de smaak uit de automaat, dat nemen we maar op de koop toe!

Daarnaast wil ik ook mijn (oud)collega's CF van de landelijke Diëtisten werkgroep die Volwassenen met Cystic Fibrosis behandelen (NVD - DVCF werkgroep) vermelden. Lidia, Sigrid, Liesbeth, Inge, Ageeth, Ingrid, Vronica, Jasmijn, Nicole, veel dank voor jullie enthousiasme voor mijn (poster)presentaties en publicaties. Onze halfjaarlijkse meetings in het UMCU zijn al vele jaren een inspirerende 'voedingsbodem' om kennis en kunde binnen CF zorg te delen. Een werkgroep waar we trots op kunnen zijn! Met deze uitwisseling en discussie over 
voedingsgerelateerde onderwerpen is de CF voedingszorg landelijk 'evidence based' op de kaart gezet en willen CF kinderdiëtetiek collega's Leonie en Marianne inmiddels aansluiten!

Also, many thanks to my international colleagues, all working in CF research and CF care! Special thanks to Susannah King, with respect to your research work, it was so great to be invited to the Australian CF Conference in 2015 as invited speaker. Such an overwhelming experience, I have enjoyed the conference and your amazing country! Furthermore, thanks to the European Nutrition Group for inviting me to talk about my research results many times. It is a great pleasure to contribute to the Masterclass Nutrition at the European Cystic Fibrosis Conference the last 2 years: Chris Smith, Dimitri Declerq, Helen White, you are all so inspiring to work with! And special thanks to my course trainer Dianna Beaufort and dear Sheralyn Orpwood; my 'Academic Writing in English' has improved with your instructions!

Lieve paranimfen, Lieve Lucienne, al meer dan 30 jaar zijn we bevriend, na een ontmoeting op de 'werkvloer' in het UMCU waar we allebei werken. Veel lief en leed hebben we met elkaar gedeeld. Zo fijn dat je een van mijn paranimfen wilt zijn en bedankt voor je mooie vriendschap al vele jaren. Je bent een geweldige doorzetter, een eigenschap die we ook delen! Lieve Jolanda, wat ben ik blij dat ook jij de rol van paranimf op je wilde nemen. Je heldere kijk op omstandigheden en mensen hebben mij geleerd te geloven in zaken die er echt toe doen. Te geloven in je jezelf en het beste tot uiting te brengen met persoonlijke aandacht voor een ieder om je heen. Dank voor je geweldige steun en betrokkenheid!

Lieve vriendinnen, vrienden en buren, Mariette, Corine, Coriene, Ariane, Lucienne, Nella, Dick, Jannie, René, Miranda, Erwin, Margo, Ruud, Sieta, Hans, Gonnie, Mario, Jojanneke, Niels, Monique, Pitrik, Manon, dank voor jullie interesse en belangstelling de afgelopen jaren voor mijn onderzoeksactiviteiten! Regelmatig werd er aan mij gevraagd, naar welk mooi land mag je nu weer naar toe om een presentatie te geven? Wat een leuke baan heb jij zeg! En ja, dat klopt helemaal! Ook dank aan mijn tennisgroep op de dinsdagmorgen. Jolanda, Irene, Dave en Robin, deze ochtend is voor mij al vele jaren een vast moment van ontspanning om naast leuke maar tijdrovende werkzaamheden te kunnen genieten van een uitdagende tenniswedstrijd en het hoofd even helemaal leeg te maken! Via de burengroepsapp werd zelfs om de link naar het lezen van de publicaties gevraagd: dank voor jullie belangstelling en betrokkenheid Tjimkje, Coen, Iris, Rolf, Esther, Fred, Dicole, Jane en Arie!

Mijn lieve gezin, Lieve Hans, veel tijd heb je mij achter de PC thuis bezig gezien. Je hebt mij altijd door dik en dun gesteund om mijn tijd te kunnen nemen bij het volgen van scholingen, 


\section{Chapter 9}

cursussen, het schrijven van artikelen en het maken van presentaties. Ook al weet ik dat je vond dat ik er vaak teveel tijd aan besteedde. Maar mijn doel was en is om altijd voor het mooiste resultaat te gaan. Jij wist jezelf gelukkig goed bezig te houden met de kinderen, golf, tennis en de laatste tijd tuinieren. Nu zullen we meer tijd samen hebben en wie weet is mijn nieuwe uitdaging jou te evenaren in je golf handicap! Veel dank lieverd, voor je geduld, rust, steun en relativeringsvermogen.

Lieve Laura, Julia en David, jullie zijn mijn trots! leder met een eigen karakter maar ook de positieve eigenschappen om voor studie en sport volledige inzet te hebben. Daarnaast zijn jullie mooie sociaal betrokken jong volwassenen, ieder met een unieke persoonlijkheid. Laat inspiratie en passie altijd de drijfveer zijn voor alles wat jullie doen in jullie leven! Ik hoop dat ik jullie hierin kan blijven steunen, een goed voorbeeld kan zijn en wil jullie bedanken voor jullie geduld en liefde. Een mooie kaart op Moederdag van jullie met trotse woorden maakt dat ik bovenal moeder ben en blijf naast mijn werk. Hiervoor ben ik heel dankbaar!

Heel erg bedankt iedereen!

Francis 


\section{CURRICULUM VITAE}

Op 3 april 1965 is Francis geboren in Ridderkerk. Hier heeft zij haar jeugd doorgebracht samen met haar broer Kees en haar ouders Gerrit en Toni Kraaijeveld. Zij heeft het VWO doorlopen op de Guillaume Farel in Ridderkerk met het zogenaamde B pakket (scheikunde, wiskunde, biologie en natuurkunde) en dit diploma behaald in 1983. Hierna heeft zij de opleiding Voeding en Dietetiek in Den Haag gevolgd aan de

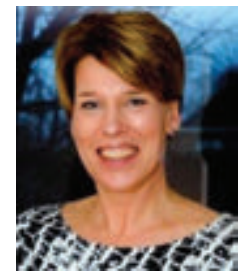
Academie De Laan. In juni 1987 heeft zij hiervan haar diploma gehaald en direct na haar opleiding in oktober 1987 is zij gestart in het UMCU bij de afdeling Dietetiek. Tijdens haar stageperiode in het UMCU kwam zij al in contact met diëtisten die ook werkzaam waren binnen voedingsonderzoek op de zogenaamde 'Balans' afdeling. In het eerste jaar van haar werkzaamheden heeft zij meegewerkt aan een onderzoek bij diabetes patiënten en parttime gewerkt om ook hiernaast de vervolgopleiding Sport en Voeding in Amsterdam te volgen. Hierna binnen het UMCU, na de verhuizing van de binnenstad naar de Uithof, bijna fulltime binnen de Divisie Hart en Longen gewerkt. Zij was toen al als teamlid/diëtist bij diverse multidisciplinaire behandelteams betrokken waaronder het harttransplantatie (HTX) team en heeft zich later meer gespecialiseerd naar in het bijzonder cystic fibrosis (CF) en longtransplantatie (LOTX). Al vele jaren met enthousiasme werkzaam en hierbij paramedische werkzaamheden gecombineerd met onderzoeksuren. Hierbij ook scholingen gevolgd om haar wetenschappelijke kennis te vergroten en zelfstandig onderzoek te mogen uitvoeren. Haar enthousiasme om te presenteren en onderzoeksresultaten te publiceren heeft geleid tot uitnodigingen niet alleen nationaal bij de NCFS (Nederlandse Cystic Fibrosis Stichting) maar ook internationaal. Met hierbij als hoogtepunt te worden uitgenodigd als 'invited speaker' op het Australische CF congres 2015 waarbij als dank een mooi schilderij van Bondi Beach Sydney ontvangen is en op haar werkkamer hangt. Ook het leveren van diverse bijdragen van (poster)presentaties op Europese CF congressen de afgelopen jaren en het verzorgen van bijdragen aan Masterclass Nutrition vindt zij heel inspirerend om te mogen doen. Een prachtige ervaring was ook het winnen van de 'Best Poster Award Section Gastro-enterology/Liver/Endocrine' 2018 in Belgrado, getiteld 'Bio-electrical impedance analysis (BIA) and relationship with pulmonary function in adult cystic fibrosis (CF) patients'. Zij vindt het tevens uitdagend om studenten van de WUR te begeleiden in het uitvoeren van wetenschappelijk voedingsonderzoek in de (poli)klinische setting. Een uitdaging die zij hoopt nog vele jaren te kunnen continueren mogelijk ook binnen nieuwe samenwerkingsverbanden 
met bijvoorbeeld studenten farmacie of geneeskunde. Zij heeft het promotietraject afgerond in het UMCU onder supervisie van Prof Harry Heijerman in samenwerking met Dr. Nicole de Roos van de WUR.

Naast haar werkzaamheden in het UMCU is Francis 25 jaar getrouwd met Hans Hollander, heeft zij 3 prachtige kinderen Laura, en de tweeling Julia en David. Het combineren van haar werk en gezin is mede mogelijk geweest dankzij de hulp van haar trouwe oppas Jetty, haar moeder Toni en schoonouders (Gerrit en Maja). Haar ouders zijn helaas niet meer in leven maar hebben haar altijd geïnspireerd te studeren en waren meelevend in alle activiteiten die zij ondernomen heeft. Inmiddels studeren haar kinderen in uiteenlopende richtingen zoals strafrecht, farmacie en ICT. 




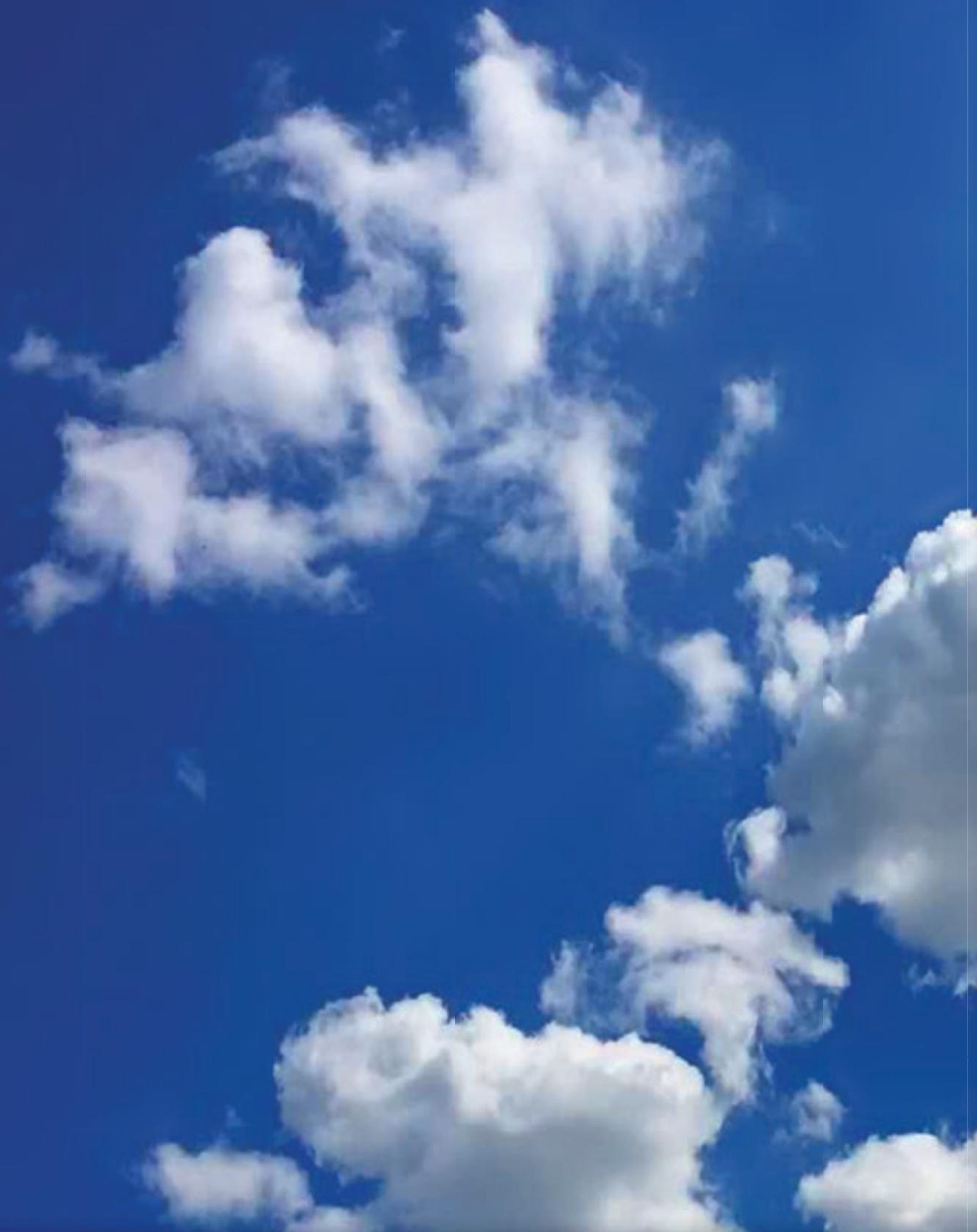

